\title{
Conformal four-point correlation functions from the operator product expansion
}

\author{
Jean-François Fortin, ${ }^{a}$ Valentina Prilepina ${ }^{a}$ and Witold Skiba ${ }^{b}$ \\ ${ }^{a}$ Département de Physique, de Génie Physique et d'Optique, Université Laval, \\ Québec, QC G1V 0A6, Canada \\ ${ }^{b}$ Department of Physics, Yale University, \\ New Haven, CT 06520, U.S.A. \\ E-mail: jean-francois.fortin@phy.ulaval.ca, \\ valentina.prilepina.1@ulaval.ca, witold.skiba@yale.edu
}

ABSTRACT: We show how to compute conformal blocks of operators in arbitrary Lorentz representations using the formalism described in $[1,2]$ and present several explicit examples of blocks derived via this method. The procedure for obtaining the blocks has been reduced to (1) determining the relevant group theoretic structures and (2) applying appropriate predetermined substitution rules. The most transparent expressions for the blocks we find are expressed in terms of specific substitutions on the Gegenbauer polynomials. In our examples, we study operators which transform as scalars, symmetric tensors, two-index antisymmetric tensors, as well as mixed representations of the Lorentz group.

KeYwords: Conformal Field Theory, Conformal and W Symmetry

ARXIV EPRINT: 1907.10506 


\section{Contents}

1 Introduction 1

2 Overview of methods and results 3

3 Four-point correlation functions $\quad 5$

3.1 OPE and four-point correlation functions 6

$\begin{array}{lll}3.2 & \text { Three-point tensorial function } & 7\end{array}$

3.3 Rules for four-point correlation functions 8

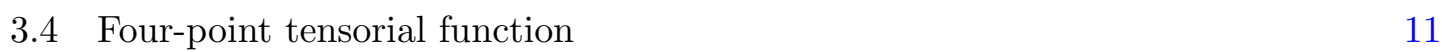

4 Examples of four-point correlation functions $\quad 12$

$\begin{array}{lll}4.1 & \text { Pre-conformal blocks } & 12\end{array}$

$\begin{array}{lll}\text { 4.1.1 Symmetric-traceless exchange } & 12\end{array}$

$\begin{array}{lll}\text { 4.1.2 } \ell \boldsymbol{e}_{1}+\boldsymbol{e}_{2} \text { exchange } & 14\end{array}$

$\begin{array}{ll}4.2 \text { Conformal blocks and four-point correlation functions } & 15\end{array}$

4.2.1 Symmetric-traceless exchange in scalar-scalar-scalar-scalar $\quad 16$

4.2.2 Symmetric-traceless exchange in scalar-scalar-scalar- $\boldsymbol{e}_{2} \quad 21$

4.2.3 Symmetric-traceless exchange in scalar-vector-scalar-vector 23

4.2.4 $\boldsymbol{l} \boldsymbol{e}_{1}+\boldsymbol{e}_{2}$ exchange in scalar-vector-scalar-vector 28

4.2.5 Symmetric-traceless exchange in scalar-scalar-vector-vector $\quad 29$

4.2.6 Conformal blocks as linear combinations of Gegenbauer polynomials with substitutions $\quad 30$

5 Discussion and conclusion $\quad 31$

\section{Introduction}

Applications of conformal field theory (CFT) in high-energy and condensed matter physics are well-known, as is the connection between gravity and CFTs. Motivation for renewed interest in CFTs includes the plethora of fruitful advances of the bootstrap program [3,4] in more than two dimensions. (The modern bootstrap literature is vast. It spans many interesting numerical results [5-44], a variety of impressive analytic results [45-73], work involving global symmetries [74-93] and higher-spin fields [94-99], as well as lectures and reviews [100-103].) The starting point for the bootstrap are the conformal blocks, which are the building blocks of the four-point correlation functions. Calculating conformal blocks beyond two dimensions has proved daunting, and only a few cases were successfully worked out almost twenty years ago [104, 105] (see also [106-109] for earlier work). With the revival of interest in the conformal bootstrap, several new results for conformal blocks were developed more recently [110-159] using a variety of different methods. 
A different approach for the computation of conformal blocks was recently proposed in $[1,2]$. It relies on using the operator product expansion (OPE) in the embedding space [160-163]. The framework for embedding space OPE was introduced in [164-169], with further developments presented in [170-172]. This approach can be applied to yield any conformal block in general spacetime dimensions. In this formalism, operators in arbitrary Lorentz representations are uplifted to the embedding space in a uniform manner using products of spinor representations alone. Derivatives naturally occur in the OPE, and hence it is of interest to fully determine their action in order to directly obtain the blocks. These were evaluated explicitly in $[1,2]$ for any expression that may potentially arise in any $M$-point function. With the action of derivatives already in hand, computing conformal blocks just requires finding the projection operators for irreducible Lorentz representations and then performing appropriate replacements of terms with the corresponding expressions obtained from derivatives in the OPE.

In this work, we derive several four-point conformal blocks using the approach developed in $[1,2]$. We have two main goals here. One is to illustrate how the formalism performs in practice. Another is to validate the approach by comparing the results with the existing ones in the literature whenever available. Some of the ingredients needed here, in particular, the projection operators and three-point tensor structures, were studied in detail in $[173,174]$; we rely on those results in this paper.

An interesting aspect of the present approach is that all conformal blocks computed here can be expressed in terms of the Gegenbauer polynomials onto which particular substitution rules are then applied. The Gegenbauer polynomials are functions of a variable $X$, and a set of substitution rules transforms $X$ into the final answer.

This paper is organized as follows: we start with an overview of our method and main results in section 2. Section 3 expresses all four-point correlation functions in terms of the conformal blocks. The conformal blocks themselves are obtained by contracting two tensor structures, each originating from the OPE, with the so-called "pre-conformal blocks". These pre-conformal blocks depend primarily on the Lorentz quantum numbers of the exchanged quasi-primary operator. They are computed in two steps using the corresponding hatted projection operators. In the first step, the projection operators are transformed using the three-point tensorial function. In the second step, the result is transformed further by a four-point conformal substitution rule yielding the proper conformal quantity. The resulting pre-conformal blocks are linear combinations of tensorial objects, which involve the generalized Exton $G$-functions of the conformal cross-ratios. The contractions of the pre-conformal blocks with the two tensor structures can be facilitated with the help of several contiguous relations, leading to the standard conformal blocks. In this work, all pre-conformal blocks and conformal blocks are computed in the $s$-channel. Section 4 illustrates how the formalism can be applied to derive pre-conformal blocks and conformal blocks in a series of examples. The conformal blocks are all written in terms of appropriate conformal substitutions on the Gegenbauer polynomials. As such, the conformal blocks presented here are the final answers that do not contain any derivatives. Comparison with the existing literature demonstrates the validity of the approach. Finally, section 5 concludes, pointing out the importance of hatted projection operators and tensor structures 
in the computation of pre-conformal blocks and conformal blocks, respectively. The reader interested in the general method based on the OPE is referred to [1,2] for an extensive exposition of the formalism.

For certain computations, the answers are applicable in $d \geq 3$ only, since in that case extra tensor structures appear which must be taken into account appropriately. Those cases should be clear from the context. Moreover, although the formalism works for any spacetime signature, the emphasis here is on Lorentz signature.

\section{Overview of methods and results}

Here we provide an overview of the main methods used and the most important results found in this work. Readers familiar with $[1,2]$ are advised to skip directly to section 3. As the results obtained here are highly technical in nature, a bird's-eye view may be of advantage. For this, the reader is encouraged to consult this section before delving into the details of the methods and results.

Throughout, we work in the $(d+2)$-dimensional embedding space with light-cone coordinates denoted by $\eta^{A}$. The most important tool used here is the OPE described in (3.1). The fact that the OPE converges absolutely at finite separation in a CFT has the powerful consequence that it can be exploited as a tool to compute $M$-point functions in terms of $(M-1)$-point functions. Here we apply the embedding space OPE to determine four-point functions from three-point functions.

The differential operator $\mathcal{D}_{12}^{\left(d, h_{i j k}-n_{a} / 2, n_{a}\right)}$ appearing in the OPE (3.1) exhibits several useful properties explored in $[1,2]$. Notably, this operator features derivatives with respect to $\eta_{2}$ only and therefore commutes with all other coordinates. Further, the superscript label $n_{a}$ on the operator denotes the number of vector indices, which are frequently omitted whenever it is clear from the context how they are contracted. Setting $n_{a}=n$, it was found earlier that $\mathcal{D}_{12}$ satisfies the identity

$$
\mathcal{D}_{12}^{(d, h, n) A_{1} \cdots A_{n}} \eta_{2}^{A_{n+1}} \cdots \eta_{2}^{A_{n+k}}=\left(\eta_{1} \cdot \eta_{2}\right)^{\frac{k}{2}} \mathcal{D}_{12}^{(d, h, n+k) A_{1} \cdots A_{n+k}},
$$

which implies that the action of $\mathcal{D}_{12}$ on any string of coordinates $\eta_{2}$ with free Lorentz indices may be absorbed into $\mathcal{D}_{12}$ by simply shifting the index $n$ appropriately. As may be foreseen from the above property, $\mathcal{D}_{12}^{(d, h, n) A_{1} \cdots A_{n}}$ is symmetric under the interchange of any pair of indices; moreover, it is also traceless upon contraction with the metric $g_{A_{1} A_{2}}$.

What is perhaps the most consequential result of $[1,2]$ is that the action of $\mathcal{D}_{12}^{(d, h, n)}$ on an arbitrary product $\Pi_{i \neq 2}\left(\eta_{i} \cdot \eta_{2}\right)^{p_{i}}$ can be evaluated explicitly for the most general quantity appearing in $M$-point correlation functions. The expression for any CFT correlation function features coordinates with Lorentz indices that take care of the spin of a given operator and powers of $\left(\eta_{i} \cdot \eta_{j}\right)$ that account for the scaling dimension of the operator in question. Thus, $\mathcal{D}_{12}^{(d, h, n)}$ can be used to construct higher-point functions for operators in arbitrary Lorentz representations. The resulting expressions generated by the present method are naturally found in closed form with no derivatives or integrals that need to be evaluated. An $M$-point function is therefore given in terms of $\mathcal{D}_{12}^{(d, h, n)}$ acting on a function with $M-1$ points. However, the upshot is that the action of the OPE differential operator 
has been determined in complete generality. Notwithstanding, even with general formulas known, obtaining the final particular expressions in a given case can be cumbersome. This is because correlators of spinning operators often contain many terms, with each term generically associated with different indices $h$ and $n$ in $\mathcal{D}_{12}^{(d, h, n)}$, thus leading to complicated formulas. For three and four points, the relevant action of $\mathcal{D}_{12}^{(d, h, n)}$ on the coordinates is described in (3.6) and (3.16), respectively.

Another crucial ingredient of the calculations described here relates to the treatment of operators with spin. In this work, we denote Lorentz representations of $\mathrm{SO}(d)$ by their Dynkin indices $\left(N_{1}, N_{2}, \ldots, N_{r}\right)$, where $r$ is the rank of $\mathrm{SO}(d)$. Here we use $N_{1} \boldsymbol{e}_{1}+N_{2} \boldsymbol{e}_{2}+\ldots$ in place of $\left(N_{1}, N_{2}, \ldots\right)$, interchangeably. We take all operators to carry spinor indices only, as spinor representations are faithful and an arbitrary representation may be obtained from a product of spinors. Transformation properties of operators under conformal transformations are encoded by the half-projectors $\left(\mathcal{T}_{12}^{N} \Gamma\right)$ appearing in the OPE and correlation functions, for instance in (3.1) and (3.2), where $\boldsymbol{N}$ denotes the representation of a given operator. The half-projectors are present to translate objects from products of spinor indices into appropriate combinations of vector indices with a given symmetry under permutations if the representation is bosonic and a combination of vector indices and one spinor index if the representation is fermionic. The half-projectors play essentially spectator roles and are in place to ensure that all expressions transform properly. The more conventional expressions with operators carrying vector indices instead of spinor indices can be obtained trivially by contracting the operators with half-projectors, for example $\left(\mathcal{T}_{N} \Gamma\right) * \mathcal{O}^{N}$, where the $*$ denotes the full contraction of the spinor indices.

As mentioned, the half-projectors serve the function of group-theoretic bookkeeping for the external operators. The half-projectors square to form projection operators, denoted by $\hat{\mathcal{P}}^{N}$, hence the half-projector terminology. The projectors act on the vector indices for bosonic representations, or vector indices and one spinor index for fermionic ones, given a specific representation $\boldsymbol{N}$. The projectors appear in our formulas for the exchange operators, for example in (3.13).

The central expression that leads directly to the four-point blocks is (3.13). This result is applicable to exchanged or external operators of any Lorentz representation. The expression for $\bar{J}$ illustrates how the invocation of the OPE, and specifically the action of the derivative $\mathcal{D}_{12}$, are applied in practice. One needs to keep track of the different ways in which $\eta_{2}$ appears in the expression. In particular, it may carry free Lorentz indices, denoted by $\left(\bar{\eta}_{2}\right)^{s_{2}}$, or be found in dot products present inside the conformal cross ratios denoted $x_{3}$ and $x_{4}$. Each individual power of $\left(\bar{\eta}_{2}\right)^{s_{2}} x_{3}^{r_{3}} x_{4}^{r_{4}}$ is accordingly replaced by the corresponding $\bar{I}_{12 ; 34}$ function that was obtained from the OPE in $[1,2]$. In this fashion, the computation of the blocks has been reduced to substitutions and bookkeeping. Obtaining the final expressions for the blocks necessitates contracting the indices on $\bar{J}$ with the representations of the external operators. This is accomplished by contraction with the group-theoretic structures ${ }_{a} t_{i j}^{12 m}$ and ${ }_{b} t_{k l m}^{34}$, which ultimately leads to the result in (3.14).

Let us now give an overview of the concrete examples considered in this paper. Here we work out five distinct sets of conformal blocks with the following choices of external operators: four scalars, three scalars and an antisymmetric tensor, two scalars and two 
vectors. In all cases, the exchanged operators are either the $\ell$-index traceless symmetric tensor or in the mixed $\ell \boldsymbol{e}_{1}+\boldsymbol{e}_{2}$ representation. From the perspective of our framework, we have found that the most convenient way to express the blocks is in terms of substitution rules on the Gegenbauer polynomials.

The simplest example of this is the scalar block with symmetric traceless tensor exchange $\ell \boldsymbol{e}_{1}$. The expression for the block, (4.13), contains a purely numerical normalization factor $\omega$ and the $\ell$-th Gegenbauer polynomial $C_{\ell}^{(d / 2-1)}(X)$ of weight $d / 2-1$ that depends on the conformal cross-ratios. This expression completely encodes the block when combined with the associated substitution rule. Here the special variable $X=$ $\frac{1}{2}\left[\left(\alpha_{4}-\alpha_{3}\right) x_{4}-\left(\alpha_{3}-\alpha_{2}\right) x_{3}\right]$ depends on the cross-ratios $x_{3}=\frac{u}{v}$ and $x_{4}=u$ and on placeholder variables $\alpha_{2,3,4}$. Once the Gegenbauer polynomial has been expanded in terms of powers of $X$ and in turn $X$ is expressed in terms of $\alpha_{2,3,4}$ and $x_{3,4}$ one obtains a finite power series $C_{\ell}^{(d / 2-1)}(X)=\sum c_{\ell ; s_{2}, s_{3}, s_{4}, r_{3}, r_{4}} \alpha_{2}^{s_{2}} \alpha_{3}^{s_{3}} \alpha_{4}^{s_{4}} x_{3}^{r_{3}} x_{4}^{r_{4}}$. Each term in the series is replaced by the function $\bar{I}_{12 ; 34}$, whose arguments depend on the powers $s_{2,3,4}$ and $r_{3,4}$. The expression obtained in this way agrees directly with [119]; moreover, the recursion relation (4.16), given in [104], is satisfied.

All other blocks exhibit very similar, albeit more involved, structures. The dependence on the conformal cross-ratios is given in terms of the Gegenbauer polynomials, see for example (4.26) and (4.27). These encode some of the blocks for the symmetric tensor $\ell \boldsymbol{e}_{1}$ exchange with the scalar-vector-scalar-vector external operators. The meaning of these expressions is exactly the same as before. The block in (4.26) consists of two Gegenbauer polynomials, while the one in (4.27) of four polynomials. Every one of the Gegenbauer polynomials is associated with a substitution rule that specifies the expressions corresponding to powers of $\alpha_{2,3,4}$ and $x_{3,4}$ upon expansion of $X$. The crux of the calculation involves finding the Gegenbauer polynomials and their associated substitution rules. We do not find it useful, other than for direct comparison with the literature, to expand the expressions completely, as this is straightforward. In the context of our formalism, conformal blocks given in terms of the Gegenbauer polynomials and the associated substitution rules are in fact the final expressions.

In practice, we have found it most convenient to work with what we term the "mixed basis." Oftentimes, we use the OPE to obtain four-point functions from the three-point ones but do not exploit the OPE directly to obtain the three-point from the two-point functions. The price of convenience is that we end up with one basis of group-theoretic structures for the OPE and another basis for the three-point functions. The mixed basis blocks are denoted with mixed brackets, for example $\mathscr{G}_{(a \mid b]}^{N}$. The transformation from one basis to another are linear and are discussed in section 4.2 .

\section{Four-point correlation functions}

In this section, we compute four-point correlation functions in the embedding space with the help of the OPE, as laid out in $[1,2]$. The procedure is analogous to the one used to obtain three-point correlation functions from the OPE [174]. The result combines a group-theoretic part, which depends on the Lorentz irreducible representation of the exchanged quasi-primary operator, and a scalar part, which involves simple powers of the 
conformal cross-ratios. The latter is fixed by the conformal dimensions of the exchanged and the external quasi-primary operators. Afterward, some simple substitution rules are introduced to transform these objects into tensorial functions appearing in four-point correlation functions, namely the conformal blocks.

\subsection{OPE and four-point correlation functions}

Four-point correlation functions can be computed from the OPE $[1,2]$

$$
\begin{array}{rlrl}
\mathcal{O}_{i}\left(\eta_{1}\right) \mathcal{O}_{j}\left(\eta_{2}\right) & =\left(\mathcal{T}_{12}^{\boldsymbol{N}_{i}} \Gamma\right)\left(\mathcal{T}_{21}^{\boldsymbol{N}_{j}} \Gamma\right) \cdot \sum_{k} \sum_{a=1}^{N_{i j k}} \frac{{ }_{a} c_{i j}{ }^{k}{ }_{a} t_{i j}^{12 k}}{\left(\eta_{1} \cdot \eta_{2}\right)^{p_{i j k}}} \cdot \mathcal{D}_{12}^{\left(d, h_{i j k}-n_{a} / 2, n_{a}\right)}\left(\mathcal{T}_{12 \boldsymbol{N}_{k}} \Gamma\right) * \mathcal{O}_{k}\left(\eta_{2}\right), \\
p_{i j k} & =\frac{1}{2}\left(\tau_{i}+\tau_{j}-\tau_{k}\right), & h_{i j k} & =-\frac{1}{2}\left(\chi_{i}-\chi_{j}+\chi_{k}\right), \\
\tau_{\mathcal{O}} & =\Delta_{\mathcal{O}}-S_{\mathcal{O}}, & \chi_{\mathcal{O}} & =\Delta_{\mathcal{O}}-\xi_{\mathcal{O}}, \quad \xi_{\mathcal{O}}=S_{\mathcal{O}}-\left\lfloor S_{\mathcal{O}}\right\rfloor,
\end{array}
$$

where $\Delta_{\mathcal{O}}$ is the scaling dimension of the operator in question; $S_{\mathcal{O}}$ is the operator spin, defined to be half the number of its spinor indices; $\tau_{\mathcal{O}}$ is the twist; while $\xi_{\mathcal{O}}$ is a parameter that is either 0 for bosonic or $\frac{1}{2}$ for fermionic operators. The spin $S_{\mathcal{O}}$ is either an integer or a half-integer and does not provide a complete description of an operator representation, which can only be specified by the weights. Further, the OPE coefficients ${ }_{a} c_{i j}{ }^{k}$ are purely numerical, while the half-projectors $\mathcal{T}_{i j}^{N} \Gamma$ and the tensor ${ }_{a} t_{i j}^{12 k}$ carry Lorentz indices, as does the differential operator $\mathcal{D}_{12}$. For now, we have suppressed all Lorentz indices for brevity. They will be restored shortly, but we stress that this statement applies to arbitrary operator representations.

The OPE yields four-point correlation functions from three-point ones as

$$
\begin{aligned}
\left\langle\mathcal{O}_{i}\left(\eta_{1}\right) \mathcal{O}_{j}\left(\eta_{2}\right) \mathcal{O}_{k}\left(\eta_{3}\right) \mathcal{O}_{\ell}\left(\eta_{4}\right)\right\rangle= & \left(\mathcal{T}_{12}^{\boldsymbol{N}_{i}} \Gamma\right)\left(\mathcal{T}_{21}^{\boldsymbol{N}_{j}} \Gamma\right) \cdot \sum_{m} \sum_{a=1}^{N_{i j m}}(-1)^{2 \xi m} \frac{{ }_{a} c_{i j}{ }^{m}{ }_{a} t_{i j}^{12 m}}{\left(\eta_{1} \cdot \eta_{2}\right)^{p_{i j m}}} \\
& \cdot \mathcal{D}_{12}^{\left(d, h_{i j m}-n_{a} / 2, n_{a}\right)} \\
& \cdot\left(\mathcal{T}_{12 \boldsymbol{N}_{m}} \Gamma\right) *\left\langle\mathcal{O}_{k}\left(\eta_{3}\right) \mathcal{O}_{\ell}\left(\eta_{4}\right) \mathcal{O}_{m}\left(\eta_{2}\right)\right\rangle .
\end{aligned}
$$

Three-point correlation functions can also be obtained from the OPE (3.1), see [174], as can be the two-point correlation functions [173].

Upon inserting the result of [174] in (3.2), the four-point correlation functions assume the form

$$
\begin{aligned}
& \left\langle\mathcal{O}_{i}\left(\eta_{1}\right) \mathcal{O}_{j}\left(\eta_{2}\right) \mathcal{O}_{k}\left(\eta_{3}\right) \mathcal{O}_{l}\left(\eta_{4}\right)\right\rangle \\
& =\frac{\left(\mathcal{T}_{12}^{\boldsymbol{N}_{i}} \Gamma\right)^{\{A a\}}\left(\mathcal{T}_{21}^{\boldsymbol{N}_{j}} \Gamma\right)^{\{B b\}}\left(\mathcal{T}_{34}^{\boldsymbol{N}_{k}} \Gamma\right)^{\{C c\}}\left(\mathcal{T}_{43}^{\boldsymbol{N}_{l}} \Gamma\right)^{\{D d\}}}{\left(\eta_{1} \cdot \eta_{2}\right)^{\frac{1}{2}\left(\tau_{i}-\chi_{i}+\tau_{j}+\chi_{j}\right)}\left(\eta_{1} \cdot \eta_{3}\right)^{\frac{1}{2}\left(\chi_{i}-\chi_{j}+\chi_{k}-\chi_{l}\right)}\left(\eta_{1} \cdot \eta_{4}\right)^{\frac{1}{2}\left(\chi_{i}-\chi_{j}-\chi_{k}+\chi_{l}\right)}\left(\eta_{3} \cdot \eta_{4}\right)^{\frac{1}{2}\left(-\chi_{i}+\chi_{j}+\tau_{k}+\tau_{l}\right)}} \\
& \times \sum_{m} \sum_{a=1}^{N_{i j m}} \sum_{b=1}^{N_{k l m}}(-1)^{2 \xi_{m}} \lambda_{N_{m} a} c_{i j}{ }^{m} b c_{k l m}\left({ }_{a} t_{i j}^{12 m}\right)_{\{a A\}\{b B\}}{ }^{\{E e\}\{F\}}\left({ }_{b} t_{k l m}^{34}\right)_{\{c C\}}\{d D\}\left\{e^{\prime} E^{\prime}\right\}\left\{F^{\prime}\right\} \\
& \times\left[\frac{\left(\eta_{1} \cdot \eta_{2}\right)\left(\eta_{3} \cdot \eta_{4}\right)}{\left(\eta_{1} \cdot \eta_{3}\right)\left(\eta_{1} \cdot \eta_{4}\right)}\right]^{h_{i j m}} \mathcal{D}_{12\{F\}}^{\left(d, h_{i j m}-n_{a} / 2, n_{a}\right)}\left[\frac{\left(\eta_{1} \cdot \eta_{2}\right)\left(\eta_{3} \cdot \eta_{4}\right)}{\left(\eta_{1} \cdot \eta_{4}\right)\left(\eta_{2} \cdot \eta_{3}\right)}\right]^{-h_{k l m}}\left[\frac{\left(\eta_{1} \cdot \eta_{2}\right)\left(\eta_{3} \cdot \eta_{4}\right)}{\left(\eta_{1} \cdot \eta_{3}\right)\left(\eta_{2} \cdot \eta_{4}\right)}\right]^{-h_{l k m}} \\
& \times\left(\frac{\eta_{2} \cdot \Gamma \hat{\mathcal{P}}_{21}^{N_{m}} \cdot \hat{\mathcal{P}}_{23}^{N_{m}} \eta_{3} \cdot \Gamma}{\left(\eta_{2} \cdot \eta_{3}\right)}\right)_{\{e E\}}^{\left\{E^{\prime \prime} e^{\prime \prime}\right\}}\left(\bar{J}_{34 ; 2}^{\left(d, h_{k l m}, n_{b}, \Delta_{m}, \boldsymbol{N}_{m}\right)}\right)_{\left\{e^{\prime \prime} E^{\prime \prime}\right\}}\left\{E^{\prime} e^{\prime}\right\}\left\{F^{\prime}\right\},
\end{aligned}
$$


where the three-point correlation function quantities are

$$
{ }_{a} c_{i j k}=\sum_{l}{ }_{a} c_{i j}{ }^{l} c_{l k} \mathbb{1}, \quad{ }_{a} t_{i j k}^{12}={ }_{a} t_{i j}^{12 k^{C}}\left[\left(C_{\Gamma}^{-1}\right)\right]^{2 \xi_{k}}(g)^{n_{v}^{k}}(g)^{n_{a}}
$$

and $\lambda_{N_{k}}$ is a normalization constant chosen such that the two-point tensor structures are orthonormalized [173]. The sets of Lorentz/spinor (upper/lower case, respectively) indices $\{A a\},\{B b\},\{C c\},\{D d\}$ correspond to the external operators, the indices $\{E e\}$ to the exchanged operator, while the set $\{F\}$ is associated with the OPE differential operator $\mathcal{D}_{12}$ in (3.1). Both primed and unprimed indices appear for $\{E e\}$ and $\{F\}$, as the projector operators $\mathcal{P}_{i j}^{\boldsymbol{N}_{m}}$ carry both upper and lower indices. We should note that the tensor structures ${ }_{a} t_{i j}^{12 m}$ and ${ }_{b} t_{k l m}^{34}$ serve to contract the indices among the half-projectors $\mathcal{T}_{i j}^{N} \Gamma$ and the projectors $\mathcal{P}_{i j}^{N_{m}}$ to the representation of the exchanged operator and the differential operator. There are no restrictions on the Lorentz representations of any operators in (3.3).

Before delving into a discussion of the conformal substitution rule, we find it necessary to explicitly display the three-point tensorial function.

\subsection{Three-point tensorial function}

In (3.3), the three-point tensorial quantity $\overline{\bar{J}}_{34 ; 2}^{(d, h, n, \Delta, N)}$ is known from the three-point correlation functions [174] and is obtained by a simple conformal substitution, namely ${ }^{1}$

$$
\begin{aligned}
& \overline{\bar{J}}_{34 ; 2}^{(d, h, n, \Delta, \boldsymbol{N})}=\left(\overline{\bar{\eta}}_{2} \cdot \Gamma \hat{\mathcal{P}}_{24}^{N} \cdot \hat{\mathcal{P}}_{34}^{N} \overline{\bar{\eta}}_{4} \cdot \Gamma\right)_{c s_{3}}
\end{aligned}
$$

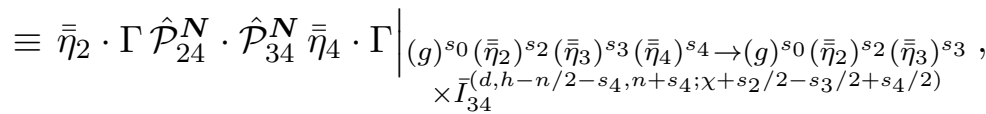

where the metric $g$, and coordinates $\overline{\bar{\eta}}_{i}$ carry Lorentz indices, but we have only exhibited their total numbers denoted by the powers $s_{0,2,3,4}$. The three-point tensorial function is

$$
\bar{I}_{34}^{(d, h, n ; p)}=\sum_{\substack{q_{0}, q_{2}, q_{3}, q_{4} \geq 0 \\ \bar{q}=2 q_{0}+q_{2}+q_{3}+q_{4}=n}} S_{\left(q_{0}, q_{2}, q_{3}, q_{4}\right)} \rho^{(d, h ; p)} K^{\left(d, h ; p ; q_{0}, q_{3}, q_{4}, q_{2}\right)},
$$

and it is obtained from a general $M$-point result in [1,2].

The totally symmetric tensor, the prefactor and the $K$-function appearing in (3.6) are

$$
\begin{aligned}
& S_{\left(q_{0}, q_{2}, q_{3}, q_{4}\right)}^{A_{1} \cdots A_{\bar{q}}}=g^{\left(A_{1} A_{2}\right.} \cdots g^{A_{2 q_{0}-1} A_{2 q_{0}}} \overline{\bar{\eta}}_{2}^{A_{2 q_{0}+1}} \cdots \overline{\bar{\eta}}_{2}^{A_{2 q_{0}+q_{2}}}
\end{aligned}
$$

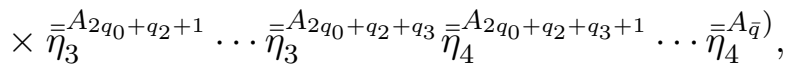

$$
\begin{aligned}
& \rho^{(d, h ; p)}=(-2)^{h}(p)_{h}(p+1-d / 2)_{h}, \\
& K^{\left(d, h ; p ; q_{0}, q_{3}, q_{4}, q_{2}\right)}=\frac{(-1)^{\bar{q}-q_{0}-q_{3}-q_{4}}(-2)^{\bar{q}-q_{0}} \bar{q} !}{q_{0} ! q_{2} ! q_{3} ! q_{4} !} \frac{(-h-\bar{q})_{\bar{q}-q_{0}-q_{4}}(p+h)_{\bar{q}-q_{0}-q_{3}}}{(p+1-d / 2)_{-q_{0}-q_{3}-q_{4}}},
\end{aligned}
$$

\footnotetext{
${ }^{1}$ Departing from the notation used in [174], homogeneized quantities for three-point correlation functions are denoted by double bars to avoid confusion with homogeneized quantities for four-point correlation functions, denoted by single bars.
} 
with $\bar{q}=2 q_{0}+q_{2}+q_{3}+q_{4}$. In the totally symmetric tensor, the homogeneized embedding space coordinates are defined as

$$
\overline{\bar{\eta}}_{i}^{A}=\frac{\left(\eta_{j} \cdot \eta_{k}\right)^{\frac{1}{2}}}{\left(\eta_{i} \cdot \eta_{j}\right)^{\frac{1}{2}}\left(\eta_{i} \cdot \eta_{k}\right)^{\frac{1}{2}}} \eta_{i}^{A},
$$

with $(i, j, k)$ a cyclic permutation of $(2,3,4)$. Clearly, the three-point tensorial function is totally symmetric and traceless with respect to the embedding space metric. As such, it satisfies the following contiguous relations $[1,2]$ :

$$
\begin{aligned}
g \cdot \bar{I}_{34}^{(d, h, n ; p)} & =0, \\
\overline{\bar{\eta}}_{3} \cdot \bar{I}_{34}^{(d, h, n ; p)} & =\bar{I}_{34}^{(d, h+1, n-1 ; p)}, \\
\overline{\bar{\eta}}_{4} \cdot \bar{I}_{34}^{(d, h, n ; p)} & =\rho^{(d, 1 ;-h-n)} \bar{I}_{34}^{(d, h, n-1 ; p)}, \\
\overline{\bar{\eta}}_{2} \cdot \bar{I}_{34}^{(d, h, n ; p)} & =\bar{I}_{34}^{(d, h+1, n-1 ; p-1)} .
\end{aligned}
$$

Since $\overline{\bar{J}}_{34 ; 2}^{(d, h, n, \Delta, N)}$ is contracted with the tensor structure ${ }_{b} t_{k \ell m}^{34}$ in (3.3) and the latter commutes through the differential operator $\mathcal{D}_{12}^{(d, h-n / 2, n)}$, the contiguous relations (3.9) can be very handy in simplifying the quantity $\overline{\bar{J}}_{34 ; 2}^{\left(d, h_{k l m}, n_{b}, \Delta_{m}, \boldsymbol{N}_{m}\right)} \cdot{ }_{b} t_{k l m}^{34}$ when computing conformal blocks. One can also express $\overline{\bar{J}}_{34 ; 2}^{\left(d, h_{k l m}, n_{b}, \Delta_{m}, \boldsymbol{N}_{m}\right)} \cdot b t_{k l m}^{34}$ in a generic basis of tensor structures by constructing it with the help of the quantities $\mathcal{A}_{34}, \epsilon_{34}, \Gamma_{34}$ and $\mathcal{A}_{34} \cdot \overline{\bar{\eta}}_{2}$.

For future convenience, we also define $\widetilde{K}^{\left(d, h ; p ; q_{0}, q_{3}, q_{4}, q_{2}\right)}=\rho^{(d, h ; p)} K^{\left(d, h ; p ; q_{0}, q_{3}, q_{4}, q_{2}\right)}$, which will appear in the construction of the pre-conformal blocks.

\subsection{Rules for four-point correlation functions}

The last two lines in (3.3) are homogeneous of degree zero in all four embedding space coordinates. Following $[1,2]$, they can be re-expressed in terms of the homogeneized embedding space coordinates

$$
\begin{array}{ll}
\bar{\eta}_{1}^{A}=\frac{\left(\eta_{3} \cdot \eta_{4}\right)^{\frac{1}{2}}}{\left(\eta_{1} \cdot \eta_{3}\right)^{\frac{1}{2}}\left(\eta_{1} \cdot \eta_{4}\right)^{\frac{1}{2}}} \eta_{1}^{A}, & \bar{\eta}_{2}^{A}=\frac{\left(\eta_{1} \cdot \eta_{3}\right)^{\frac{1}{2}}\left(\eta_{1} \cdot \eta_{4}\right)^{\frac{1}{2}}}{\left(\eta_{1} \cdot \eta_{2}\right)\left(\eta_{3} \cdot \eta_{4}\right)^{\frac{1}{2}}} \eta_{2}^{A}, \\
\bar{\eta}_{3}^{A}=\frac{\left(\eta_{1} \cdot \eta_{4}\right)^{\frac{1}{2}}}{\left(\eta_{3} \cdot \eta_{4}\right)^{\frac{1}{2}}\left(\eta_{1} \cdot \eta_{3}\right)^{\frac{1}{2}}} \eta_{3}^{A}, & \bar{\eta}_{4}^{A}=\frac{\left(\eta_{1} \cdot \eta_{3}\right)^{\frac{1}{2}}}{\left(\eta_{3} \cdot \eta_{4}\right)^{\frac{1}{2}}\left(\eta_{1} \cdot \eta_{4}\right)^{\frac{1}{2}}} \eta_{4}^{A},
\end{array}
$$

and the conformal cross-ratios

$$
x_{3}=\frac{\left(\eta_{1} \cdot \eta_{2}\right)\left(\eta_{3} \cdot \eta_{4}\right)}{\left(\eta_{1} \cdot \eta_{4}\right)\left(\eta_{2} \cdot \eta_{3}\right)}=\frac{u}{v}, \quad x_{4}=\frac{\left(\eta_{1} \cdot \eta_{2}\right)\left(\eta_{3} \cdot \eta_{4}\right)}{\left(\eta_{1} \cdot \eta_{3}\right)\left(\eta_{2} \cdot \eta_{4}\right)}=u .
$$

Hence, the last two lines of (3.3) can be represented by the following function:

$$
\begin{aligned}
\bar{J}_{34 ; 21}^{\left(d, h_{1}, n_{1}, h_{2}, n_{2}, \Delta, N\right)}= & {\left[\frac{\left(\eta_{1} \cdot \eta_{2}\right)\left(\eta_{3} \cdot \eta_{4}\right)}{\left(\eta_{1} \cdot \eta_{3}\right)\left(\eta_{1} \cdot \eta_{4}\right)}\right]^{h_{1}} \mathcal{D}_{12}^{\left(d, h_{1}-n_{1} / 2, n_{1}\right)}\left[\frac{\left(\eta_{1} \cdot \eta_{2}\right)\left(\eta_{3} \cdot \eta_{4}\right)}{\left(\eta_{1} \cdot \eta_{4}\right)\left(\eta_{2} \cdot \eta_{3}\right)}\right]^{-h_{2}} } \\
& \times\left[\frac{\left(\eta_{1} \cdot \eta_{2}\right)\left(\eta_{3} \cdot \eta_{4}\right)}{\left(\eta_{1} \cdot \eta_{3}\right)\left(\eta_{2} \cdot \eta_{4}\right)}\right]^{\chi+h_{2}}\left(\frac{\eta_{2} \cdot \Gamma \hat{\mathcal{P}}_{21}^{N} \cdot \hat{\mathcal{P}}_{23}^{N} \eta_{3} \cdot \Gamma}{\left(\eta_{2} \cdot \eta_{3}\right)}\right) \cdot \overline{\bar{J}}_{34 ; 2}^{\left(d, h_{2}, n_{2}, \Delta, \boldsymbol{N}\right)} \\
= & \overline{\mathcal{D}}_{12}^{\left(d, h_{1}-n_{1} / 2, n_{1}\right)} x_{3}^{-h_{2}} x_{4}^{\chi+h_{2}}\left(\frac{\eta_{2} \cdot \Gamma \hat{\mathcal{P}}_{21}^{N} \cdot \hat{\mathcal{P}}_{23}^{N} \eta_{3} \cdot \Gamma}{\left(\eta_{2} \cdot \eta_{3}\right)}\right) \cdot \overline{\bar{J}}_{34 ; 2}^{\left(d, h_{2}, n_{2}, \Delta, N\right)},
\end{aligned}
$$


which depends primarily on the exchanged quasi-primary operator, most importantly, on its irreducible representation $\boldsymbol{N}$ under the Lorentz group. Using the definition of the threepoint tensorial function (3.5) and the general result of $[1,2]$ for the action of the differential operator, we find that there exists a simple conformal substitution rule for (3.12), analogous to the one in the three-point case [174]. It can be explicitly and concisely given as

$$
\begin{aligned}
& \bar{J}_{34 ; 21}^{\left(d, h_{1}, n_{1}, h_{2}, n_{2}, \Delta, N\right)} \\
& =2^{2 \xi}\left(\overline{\bar{\eta}}_{2} \cdot \Gamma \hat{\mathcal{P}}_{21}^{N} \cdot \hat{\mathcal{P}}_{23}^{N} \cdot \hat{\mathcal{P}}_{24}^{N} \cdot \hat{\mathcal{P}}_{34}^{N} \overline{\bar{\eta}}_{4} \cdot \Gamma\right)_{c s_{3}, c s_{4}} \\
& \left.\equiv 2^{2 \xi}\left(\overline{\bar{\eta}}_{2} \cdot \Gamma \hat{\mathcal{P}}_{21}^{N} \cdot \hat{\mathcal{P}}_{23}^{N} \cdot \hat{\mathcal{P}}_{24}^{N} \cdot \hat{\mathcal{P}}_{34}^{N} \overline{\bar{\eta}}_{4} \cdot \Gamma\right)_{c s_{3}}\right|_{\left(\bar{\eta}_{2}\right)^{s_{2}} x_{3}^{r_{3}} x_{4}^{r_{4}} \rightarrow \bar{I}_{12 ; 34}^{\left(d, h_{1}-n_{1} / 2-s_{2}, n_{1}+s_{2} ;-h_{2}+r_{3}, \chi+h_{2}+r_{4}\right)}},
\end{aligned}
$$

where only $\hat{\mathcal{P}}_{24}^{N}$ and $\hat{\mathcal{P}}_{34}^{N}$ are expressed in terms of the homogeneized three-point embedding space coordinates (3.8) for the three-point conformal substitution (3.5). After the threepoint conformal substitution has been implemented but before the four-point one is performed, all the embedding space coordinates are re-expressed in terms of the homogeneized four-point embedding space coordinates (3.10) and the conformal cross-ratios (3.11), with the homogeneized three-point quantities (3.8) given by

$$
\overline{\bar{\eta}}_{2}=\sqrt{x_{3} x_{4}} \bar{\eta}_{2}, \quad \overline{\bar{\eta}}_{3}=\sqrt{\frac{x_{3}}{x_{4}}} \bar{\eta}_{3}, \quad \overline{\bar{\eta}}_{4}=\sqrt{\frac{x_{4}}{x_{3}}} \bar{\eta}_{4}
$$

The four-point tensorial function $\bar{I}_{12 ; 34}^{\left(d, h, n ; p_{3}, p_{4}\right)}$ appearing in the conformal substitution rule (3.3) is described in more detail below.

A few comments on (3.13) above may be useful here. As was the case for three-point functions in (3.5), this expression is valid for any operator spins. The $\bar{J}$-function can be regarded as a pre-conformal block. It depends primarily on the Lorentz group irreducible representation $\boldsymbol{N}$ of the exchanged quasi-primary operator. Any conformal block with an exchanged operator in a given representation $\boldsymbol{N}$ can be obtained by appropriate group theory contractions as described below.

The dependence on the exchange operator is clear as the substitutions are performed on a combination of the projection operators into the representation $\boldsymbol{N}$ denoted $\mathcal{P}_{i j}^{N}$. Explicit examples of projection operators are in (4.1) and (4.5). The remaining inputs that determine the $\bar{J}$-function are numerical. These are three real numbers related to the conformal dimensions of all quasi-primary operators, two integers associated with the two symmetrictraceless irreducible representations appearing in the two tensor structures described below, and the spacetime dimension. Consequently, once the irreducible representation of the exchanged quasi-primary operator is fixed, the pre-conformal blocks (i.e. the $\bar{J}$-functions) are completely determined from the corresponding hatted projection operator. ${ }^{2}$

\footnotetext{
${ }^{2}$ Hatted projection operators are discussed in [173].
} 
After using the conformal substitution rule (3.13), the four-point correlation functions (3.3) become

$$
\begin{aligned}
& \left\langle\mathcal{O}_{i}\left(\eta_{1}\right) \mathcal{O}_{j}\left(\eta_{2}\right) \mathcal{O}_{k}\left(\eta_{3}\right) \mathcal{O}_{l}\left(\eta_{4}\right)\right\rangle \\
& =\frac{\left(\mathcal{T}_{12}^{\boldsymbol{N}_{i}} \Gamma\right)^{\{A a\}}\left(\mathcal{T}_{21}^{\boldsymbol{N}_{j}} \Gamma\right)^{\{B b\}}\left(\mathcal{T}_{34}^{\boldsymbol{N}_{k}} \Gamma\right)^{\{C c\}}\left(\mathcal{T}_{43}^{\boldsymbol{N}_{l}} \Gamma\right)^{\{D d\}}}{\left(\eta_{1} \cdot \eta_{2}\right)^{\frac{1}{2}\left(\tau_{i}-\chi_{i}+\tau_{j}+\chi_{j}\right)}\left(\eta_{1} \cdot \eta_{3}\right)^{\frac{1}{2}\left(\chi_{i}-\chi_{j}+\chi_{k}-\chi_{l}\right)}\left(\eta_{1} \cdot \eta_{4}\right)^{\frac{1}{2}\left(\chi_{i}-\chi_{j}-\chi_{k}+\chi_{l}\right)}\left(\eta_{3} \cdot \eta_{4}\right)^{\frac{1}{2}\left(-\chi_{i}+\chi_{j}+\tau_{k}+\tau_{l}\right)}} \\
& \times \sum_{m} \sum_{a=1}^{N_{i j m}} \sum_{b=1}^{N_{k l m}}(-1)^{2 \xi_{m}} \lambda_{N_{m} a} c_{i j}{ }^{m}{ }_{b} c_{k l m}\left({ }_{a} t_{i j}^{12 m}\right)_{\{a A\}\{b B\}}\{E e\}\{F\}\left({ }_{b} t_{k l m}^{34}\right)_{\{c C\}\{d D\}\left\{e^{\prime} E^{\prime}\right\}\left\{F^{\prime}\right\}} \\
& \times\left(\bar{J}_{34 ; 21}^{\left(d, h_{i j m}, n_{a}, h_{k l m}, n_{b}, \Delta_{m}, \boldsymbol{N}_{m}\right)}\right)_{\{F\}\{e E\}}\left\{E^{\prime} e^{\prime}\right\}\left\{F^{\prime}\right\} .
\end{aligned}
$$

The equation above is valid for all four-point correlation functions irrespective of the irreducible representations of the quasi-primary operators. Moreover, the nontrivial part of the computation corresponds to the contraction of the hatted projection operators. The conformal substitution rule (3.13) leading to the pre-conformal blocks is trivial.

The two tensor structures, ${ }^{3}$ which dictate the two integers mentioned above, are then needed to contract the remaining dummy indices, which leads to

$$
\begin{aligned}
& \left\langle\mathcal{O}_{i}\left(\eta_{1}\right) \mathcal{O}_{j}\left(\eta_{2}\right) \mathcal{O}_{k}\left(\eta_{3}\right) \mathcal{O}_{l}\left(\eta_{4}\right)\right\rangle \\
& =\frac{\left(\mathcal{T}_{12}^{\boldsymbol{N}_{i}} \Gamma\right)^{\{A a\}}\left(\mathcal{T}_{21}^{\boldsymbol{N}_{j}} \Gamma\right)^{\{B b\}}\left(\mathcal{T}_{34}^{\boldsymbol{N}_{k}} \Gamma\right)^{\{C c\}}\left(\mathcal{T}_{43}^{\boldsymbol{N}_{l}} \Gamma\right)^{\{D d\}}}{\left(\eta_{1} \cdot \eta_{2}\right)^{\frac{1}{2}\left(\tau_{i}-\chi_{i}+\tau_{j}+\chi_{j}\right)}\left(\eta_{1} \cdot \eta_{3}\right)^{\frac{1}{2}\left(\chi_{i}-\chi_{j}+\chi_{k}-\chi_{l}\right)}\left(\eta_{1} \cdot \eta_{4}\right)^{\frac{1}{2}\left(\chi_{i}-\chi_{j}-\chi_{k}+\chi_{l}\right)}\left(\eta_{3} \cdot \eta_{4}\right)^{\frac{1}{2}\left(-\chi_{i}+\chi_{j}+\tau_{k}+\tau_{l}\right)}} \\
& \times \sum_{m} \sum_{a=1}^{N_{i j m}} \sum_{b=1}^{N_{k l m}}{ }_{a} c_{i j}{ }^{m}{ }_{b} c_{k l m}\left(\mathscr{G}_{(a \mid b)}^{i j|m| k l}\right)_{\{a A\}\{b B\}\{c C\}\{d D\}},
\end{aligned}
$$

with the conformal blocks

$$
\mathscr{G}_{(a \mid b)}^{i j|m| k l}=(-1)^{2 \xi_{m}} \lambda_{\boldsymbol{N}_{m} a} t_{i j}^{12 m} \cdot \bar{J}_{34 ; 21}^{\left(d, h_{i j m}, n_{a}, h_{k l m}, n_{b}, \Delta_{m}, \boldsymbol{N}_{m}\right)} \cdot{ }_{b} t_{k l m}^{34} .
$$

As mentioned earlier, the contiguous relations (3.9) and (3.20) can be quite helpful in computing the conformal blocks. Therefore, it might be more efficient to contract the preconformal blocks with the appropriate tensor structures before performing all conformal substitutions, which results in the expression

$$
\begin{aligned}
& \mathscr{G}_{(a \mid b)}^{i j|m| k l}= \\
& \lambda_{\boldsymbol{N}_{m} a} t_{i j}^{12 m} \cdot\left(\left(-x_{3}\right)^{2 \xi_{m}} \bar{\eta}_{2} \cdot \Gamma \hat{\mathcal{P}}_{21}^{N_{m}} \cdot \hat{\mathcal{P}}_{23}^{N_{m}} \bar{\eta}_{3} \cdot \Gamma\left(\overline{\bar{\eta}}_{2} \cdot \Gamma \hat{\mathcal{P}}_{24}^{N_{m}} \cdot \hat{\mathcal{P}}_{34}^{N_{m}} \overline{\bar{\eta}}_{4} \cdot \Gamma\right)_{c s_{3}} \cdot b t_{k l m}^{34}\right)_{c s_{4}},
\end{aligned}
$$

for the conformal blocks, with the conformal substitution rules (3.5) and (3.13), respectively.

\footnotetext{
${ }^{3}$ Tensor structures are discussed in [174].
} 


\subsection{Four-point tensorial function}

From the results of $[1,2]$, the four-point tensorial function $\bar{I}_{12 ; 34}^{\left(d, h, n ; p_{3}, p_{4}\right)}$ is given by

$$
\bar{I}_{12 ; 34}^{\left(d, h, n ; p_{3}, p_{4}\right)}=\sum_{\substack{q_{0}, q_{1}, q_{2}, q_{3}, q_{4} \geq 0 \\ \bar{q}=2 q_{0}+q_{1}+q_{2}+q_{3}+q_{4}=n}} S_{(\boldsymbol{q})} \rho^{\left(d, h ; p_{3}+p_{4}\right)} x_{3}^{p_{3}+p_{4}+h+q_{0}+q_{2}+q_{3}+q_{4}} K_{12 ; 34 ; 3}^{\left(d, h ; p_{3}, p_{4} ; q_{0}, q_{1}, q_{2}, q_{3}, q_{4}\right)}\left(x_{3} ; y_{4}\right),
$$

with the totally symmetric tensor $S_{(\boldsymbol{q})}$

$$
S_{(\boldsymbol{q})}^{A_{1} \cdots A_{\bar{q}}}=g^{\left(A_{1} A_{2}\right.} \cdots g^{A_{2 q_{0}-1} A_{2 q_{0}}} \bar{\eta}_{1}^{A_{2 q_{0}+1}} \cdots \bar{\eta}_{1}^{A_{2 q_{0}+q_{1}}} \cdots \bar{\eta}_{4}^{A_{\bar{q}-q_{4}+1}} \cdots \bar{\eta}_{4}^{\left.A_{\bar{q}}\right)},
$$

$\bar{q}=2 q_{0}+q_{1}+q_{2}+q_{3}+q_{4}$ and $y_{4}=1-x_{3} / x_{4}$.

The $K$-function is simply a shifted version of the Exton $G$-function,

$$
\begin{aligned}
K_{12 ; 34 ; 3}^{(d, h ; \boldsymbol{p} ; \boldsymbol{q})}\left(x_{3} ; y_{4}\right)= & \frac{(-1)^{q_{0}+q_{3}+q_{4}}(-2)^{\bar{q}-q_{0}} \bar{q} !}{q_{0} ! q_{1} ! q_{2} ! q_{3} ! q_{4} !} \frac{(-h-\bar{q})_{\bar{q}-q_{0}-q_{2}}\left(p_{3}\right)_{q_{3}}\left(p_{3}+p_{4}+h\right)_{\bar{q}-q_{0}-q_{1}}}{\left(p_{3}+p_{4}\right)_{q_{3}+q_{4}}\left(p_{3}+p_{4}+1-d / 2\right)_{-q_{0}-q_{1}-q_{2}}}\left(p_{4}\right)_{q_{4}} \\
& \times K_{12 ; 34 ; 3}^{\left(d+2 \bar{q}-2 q_{0}, h+q_{0}+q_{2} ; p_{3}+q_{3}, p_{4}+q_{4}\right)}\left(x_{3} ; y_{4}\right),
\end{aligned}
$$

where

$$
\begin{aligned}
K_{12 ; 34 ; 3}^{\left(d, h ; p_{3}, p_{4}\right)}\left(x_{3} ; y_{4}\right) & =\sum_{n_{4}, n_{34} \geq 0} \frac{(-h)_{n_{34}}\left(p_{3}\right)_{n_{34}}\left(p_{3}+p_{4}+h\right)_{n_{4}}}{\left(p_{3}+p_{4}\right)_{n_{4}+n_{34}}\left(p_{3}+p_{4}+1-d / 2\right)_{n_{34}}} \frac{\left(p_{4}\right)_{n_{4}}}{n_{34} !\left(n_{4}-n_{34}\right) !} y_{4}^{n_{4}}\left(\frac{x_{3}}{y_{4}}\right)^{n_{34}} \\
& =G\left(p_{4}, p_{3}+p_{4}+h, p_{3}+p_{4}+1-d / 2, p_{3}+p_{4} ; u / v, 1-1 / v\right) .
\end{aligned}
$$

Here $G(\alpha, \beta, \gamma, \delta ; x, y)$ is the usual Exton $G$-function [109], which can be expressed in terms of the well-known fourth Appel functions as [175]

$$
\begin{aligned}
G(\alpha, \beta, \gamma, \delta ; x, 1-y)= & \frac{\Gamma(\delta) \Gamma(\delta-\alpha-\beta)}{\Gamma(\delta-\alpha) \Gamma(\delta-\beta)} F_{4}(\alpha, \beta, \gamma, \alpha+\beta+1-\delta ; x, y) \\
& +\frac{\Gamma(\delta) \Gamma(\alpha+\beta-\delta)}{\Gamma(\alpha) \Gamma(\beta)} y^{\delta-\alpha-\beta} F_{4}(\delta-\alpha, \delta-\beta, \gamma, \delta-\alpha-\beta+1 ; x, y) .
\end{aligned}
$$

As was the case for the three-point tensorial function, the four-point tensorial function satisfies contiguous relations that can greatly simplify computations. They are given by

$$
\begin{aligned}
g \cdot \bar{I}_{12 ; 34}^{(d, h, n ; p)} & =0 \\
\bar{\eta}_{1} \cdot \bar{I}_{12 ; 34}^{\left(d, h, n ; p_{3}, p_{4}\right)} & =\bar{I}_{12 ; 34}^{\left(d, h+1, n-1 ; p_{3}, p_{4}\right)}, \\
\bar{\eta}_{2} \cdot \bar{I}_{12 ; 34}^{\left(d, h, n ; p_{3}, p_{4}\right)} & =\rho^{(d, 1 ;-h-n)} \bar{I}_{12 ; 34}^{\left(d, h, n-1 ; p_{3}, p_{4}\right)}, \\
\bar{\eta}_{3} \cdot \bar{I}_{12 ; 34}^{\left(d, h, n ; p_{3}, p_{4}\right)} & =\bar{I}_{12 ; 34}^{\left(d, h+1, n-1 ; p_{3}-1, p_{4}\right)} \\
\bar{\eta}_{4} \cdot \bar{I}_{12 ; 34}^{\left(d, h, n ; p_{3}, p_{4}\right)} & =\bar{I}_{12 ; 34}^{\left(d, h+1, n-1 ; p_{3}, p_{4}-1\right)} .
\end{aligned}
$$

Further details are provided in $[1,2]$.

Finally, for future convenience, we define

$$
\begin{aligned}
G_{\left(n_{1}, n_{2}, n_{3}, n_{4}, n_{5}\right) A_{1} \cdots A_{n}}^{i j|m| k l} & \rho^{\left(d,\left(\ell+s_{2}-s_{3}-s_{4}+n_{1}\right) / 2 ;-h_{i j m}-\left(\ell+n_{2}\right) / 2\right)} x_{3}^{-s_{3}} x_{4}^{-s_{4}} \\
& \times \bar{I}_{12 ; 34}^{\left(d, h_{i j m}-\left(s_{2}-s_{3}-s_{4}+n_{3}\right) / 2, n ;-h_{k l m}+\left(r_{3}-r_{4}+n_{4}\right) / 2, \chi_{m}+h_{k l m}-\left(r_{3}-r_{4}+n_{5}\right) / 2\right)}
\end{aligned}
$$

This quantity will appear frequently in the conformal substitutions for the conformal blocks, where the meaning of $\ell, s_{i}$ and $r_{i}$ will become clear. 


\section{Examples of four-point correlation functions}

In this section, we explicitly demonstrate how to compute the pre-conformal blocks and conformal blocks using the formalism introduced in [1, 2]. Examples illustrating both computational paths explained in the previous section are given: conformal blocks will be computed either directly from pre-conformal blocks or using (3.15). The advantage of the pre-conformal blocks is that they can be used in any four-point correlation function where one of the exchanged quasi-primary operators is in the appropriate irreducible representation of the Lorentz group. Moreover, they only require the knowledge of the corresponding hatted projection operator.

\section{$4.1 \quad$ Pre-conformal blocks}

The pre-conformal blocks (3.13) are some of the most fundamental objects leading to the conformal blocks. They are straightforward to compute once the corresponding hatted projection operators are known. However, due to the proliferation of indices, they are not always expressible in a manner convenient for exposition. Because the substitution rules (3.5) and (3.13) are trivial, the pre-conformal blocks can be easily generated with the help of any convenient symbolic computation program. Hence, in the following, only some simple pre-conformal blocks are shown explicitly. Once the pre-conformal block for a specific irreducible representation is known, it can subsequently be used to obtain any conformal block with the corresponding exchanged quasi-primary operator.

\subsubsection{Symmetric-traceless exchange}

Since the hatted projection operator for quasi-primary operators in the symmetric-traceless irreducible representation $\ell \boldsymbol{e}_{1}$ is

$$
\begin{aligned}
& \left(\hat{\mathcal{P}}^{\ell e_{1}}\right)_{\mu_{\ell} \cdots \mu_{1}}^{\mu_{1}^{\prime} \cdots \mu_{\ell}^{\prime}} \\
& \left.=\sum_{i=0}^{\lfloor\ell / 2\rfloor} \frac{(-\ell)_{2 i}}{2^{2 i} i !(-\ell+2-d / 2)_{i}} g_{\left(\mu_{1} \mu_{2}\right.} g^{\left(\mu_{1}^{\prime} \mu_{2}^{\prime}\right.} \cdots g_{\mu_{2 i-1} \mu_{2 i}} g^{\mu_{2 i-1}^{\prime} \mu_{2 i}^{\prime}} g_{\mu_{2 i+1}} \mu_{2 i+1}^{\prime} \cdots g_{\left.\mu_{\ell}\right)} \mu_{\ell}^{\prime}\right)
\end{aligned}
$$

the $\overline{\bar{J}}$-functions (3.5) become

$$
\begin{aligned}
\left(\overline{\bar{J}}_{34 ; 2}^{\left(d, h_{2}, n_{2}, \Delta, \mathbf{0}\right)}\right)^{\left\{F^{\prime}\right\}}= & \bar{I}_{34}^{\left(d, h_{2}-n_{2} / 2, n_{2} ; \Delta\right)\left\{F^{\prime}\right\}} \\
\left(\overline{\bar{J}}_{34 ; 2}^{\left(d, h_{2}, n_{2}, \Delta, e_{1}\right)}\right)_{E^{\prime \prime}} E^{\prime}\left\{F^{\prime}\right\}= & g_{E^{\prime \prime}} E^{\prime} \bar{I}_{34}^{\left(d, h_{2}-n_{2} / 2, n_{2} ; \Delta\right)\left\{F^{\prime}\right\}}-\overline{\bar{\eta}}_{3 E^{\prime \prime}} \bar{I}_{34}^{\left(d, h_{2}-n_{2} / 2-1, n_{2}+1 ; \Delta\right) E^{\prime}\left\{F^{\prime}\right\}} \\
& -\overline{\bar{\eta}}_{2}^{E^{\prime}} \bar{I}_{34}^{\left(d, h_{2}-n_{2} / 2-1, n_{2}+1 ; \Delta+1\right)}\left\{F^{\prime \prime}\right\} \\
& +\bar{I}_{34}^{\left(d, h_{2}-n_{2} / 2-2, n_{2}+2 ; \Delta+1\right)} E_{E^{\prime \prime}}^{E^{\prime \prime}\left\{F^{\prime}\right\}}
\end{aligned}
$$


for the irreducible representations $\mathbf{0}$ and $\boldsymbol{e}_{1}$, respectively. Then, the pre-conformal blocks (3.13) are given by

$$
\begin{aligned}
& \left(\bar{J}_{34 ; 21}^{\left(d, h_{1}, n_{1}, h_{2}, n_{2}, \Delta, \mathbf{0}\right)}\right)_{\{F\}}\left\{F^{\prime}\right\}=\left(\bar{I}_{34}^{\left(d, h_{2}-n_{2} / 2, n_{2} ; \Delta\right)\left\{F^{\prime}\right\}}\right)_{c s_{4}} \\
& =\sum_{\substack{q_{0}, q_{2}, q_{3}, q_{4} \geq 0 \\
2 q_{0}+q_{2}+q_{3}+q_{4}=n_{2}}} g^{\left(F_{1}^{\prime} F_{2}^{\prime}\right.} \cdots g^{F_{2 q_{0}-1}^{\prime} F_{2 q_{0}}^{\prime}} \bar{\eta}_{3}^{F_{2 q_{0}+1}^{\prime}} \cdots \bar{\eta}_{3}^{F_{2 q_{0}+q_{3}}^{\prime}} \bar{\eta}_{4}^{F_{2 q_{0}+q_{3}+1}^{\prime}} \cdots \bar{\eta}_{4}^{F_{2 q_{0}+q_{3}+q_{4}}^{\prime}} \\
& \times \widetilde{K}^{\left(d, h_{2}-n_{2} / 2 ; \Delta ; q_{0}, q_{3}, q_{4}, q_{2}\right)}\left(x_{3}^{\left(q_{2}+q_{3}-q_{4}\right) / 2} x_{4}^{\left(q_{2}-q_{3}+q_{4}\right) / 2} \bar{\eta}_{2}^{F_{2_{0}+q_{3}+q_{4}+1}^{\prime}} \cdots \bar{\eta}_{2}^{\left.F_{n_{2}}^{\prime}\right)}\right)_{c s_{4}} \\
& =\widetilde{K}^{\left(d, h_{2}-n_{2} / 2 ; \Delta ; 0,0,0, n_{2}\right)} \bar{I}_{12 ; 34}^{\left(d, h_{1}-n_{1} / 2-n_{2}, n_{1}+n_{2} ;-h_{2}+n_{2} / 2, \Delta+h_{2}+n_{2} / 2\right)}\left\{{ }_{\{F\}}\left\{F^{\prime}\right\},\right.
\end{aligned}
$$

for scalar exchange and

$$
\begin{aligned}
\left(\bar{J}_{34 ; 21}^{\left(d, h_{1}, n_{1}, h_{2}, n_{2}, \Delta, e_{1}\right)}\right)_{\{F\} E}^{E^{\prime}\left\{F^{\prime}\right\}}= & \left(\mathcal{A}_{123 E} E^{\prime} \bar{I}_{34}^{\left(d, h_{2}-n_{2} / 2, n_{2} ; \Delta\right)\left\{F^{\prime}\right\}}\right)_{c s_{4}} \\
& -\left(\sqrt{x_{3} x_{4}} \bar{\eta}_{2}^{E^{\prime}} \mathcal{A}_{123 E} E^{E^{\prime \prime}} \bar{I}_{34}^{\left(d, h_{2}-n_{2} / 2-1, n_{2}+1 ; \Delta+1\right){ }_{E^{\prime \prime}}{ }^{\prime \prime} F_{c s_{4}}}\right. \\
& +\left(\mathcal{A}_{123 E} E^{\prime \prime} \bar{I}_{34}^{\left(d, h_{2}-n_{2} / 2-2, n_{2}+2 ; \Delta+1\right) E_{E^{\prime \prime}} E^{\prime}\left\{F^{\prime}\right\}}\right)_{c s_{4}},
\end{aligned}
$$

or, more explicitly,

$$
\begin{aligned}
& \left(\bar{J}_{34 ; 21}^{\left(d, h_{1}, n_{1}, h_{2}, n_{2}, \Delta, e_{1}\right)}\right)_{\{F\} E} E^{\prime}\left\{F^{\prime}\right\} \\
& =\left[\widetilde{K}^{\left(d, h_{2}-n_{2} / 2 ; \Delta ; 0,0,0, n_{2}\right)}+\frac{2 \widetilde{K}^{\left(d, h_{2}-n_{2} / 2-2 ; \Delta+1 ; 1,0,0, n_{2}\right)}}{\left(n_{2}+2\right)\left(n_{2}+1\right)}\right] \\
& \times\left[g_{E}{ }^{\prime} \bar{I}_{12 ; 34}^{\left(d, h_{1}-n_{1} / 2-n_{2}, n_{1}+n_{2} ;-h_{2}+n_{2} / 2, \Delta+h_{2}+n_{2} / 2\right)}\{F\} \quad\left\{F^{\prime}\right\}\right.
\end{aligned}
$$

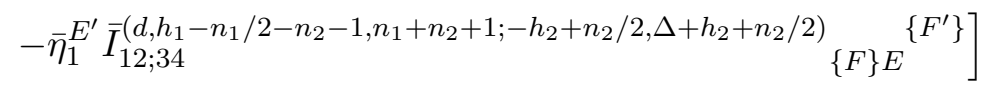

$$
\begin{aligned}
& -\left[\widetilde{K}^{\left(d, h_{2}-n_{2} / 2-1 ; \Delta ; 0,0,0, n_{2}+1\right)}+\frac{\widetilde{K}^{\left(d, h_{2}-n_{2} / 2-1 ; \Delta+1 ; 0,1,0, n_{2}\right)}}{n_{2}+1}-\frac{\widetilde{K}^{\left(d, h_{2}-n_{2} / 2-2 ; \Delta+1 ; 0,1,0, n_{2}+1\right)}}{n_{2}+2}\right] \\
& \times\left[\bar{\eta}_{3 E} \bar{I}_{12 ; 34}^{\left(d, h_{1}-n_{1} / 2-n_{2}-1, n_{1}+n_{2}+1 ;-h_{2}+n_{2} / 2+1, \Delta+h_{2}+n_{2} / 2\right)} \underset{\{F\}}{E^{\prime}\left\{F^{\prime}\right\}}\right.
\end{aligned}
$$

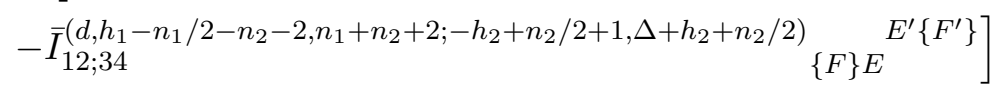

$$
\begin{aligned}
& -\left[\frac{\widetilde{K}^{\left(d, h_{2}-n_{2} / 2-1 ; \Delta+1 ; 0,0,1, n_{2}\right)}}{n_{2}+1}-\frac{\widetilde{K}^{\left(d, h_{2}-n_{2} / 2-2 ; \Delta+1 ; 0,0,1, n_{2}+1\right)}}{n_{2}+2}\right] \\
& \times\left[\bar{\eta}_{4 E} \bar{I}_{12 ; 34}^{\left(d, h_{1}-n_{1} / 2-n_{2}-1, n_{1}+n_{2}+1 ;-h_{2}+n_{2} / 2, \Delta+h_{2}+n_{2} / 2+1\right)}{ }_{\{F\}} E^{\prime}\left\{F^{\prime}\right\}\right.
\end{aligned}
$$

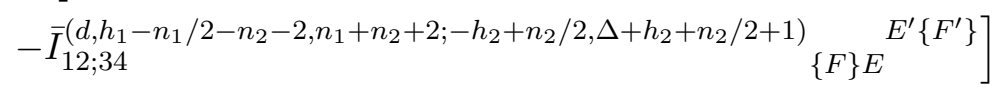

$$
\begin{aligned}
& -\left[\frac{2 \widetilde{K}^{\left(d, h_{2}-n_{2} / 2-1 ; \Delta ; 1,0,0, n_{2}-1\right)}}{n_{2}+1}-\frac{2 \widetilde{K}^{\left(d, h_{2}-n_{2} / 2-2 ; \Delta+1 ; 1,1,0, n_{2}-1\right)}}{\left(n_{2}+2\right)\left(n_{2}+1\right)}\right] \\
& \times\left[\bar{\eta}_{3 E} g^{E^{\prime}\left(F_{1}^{\prime}\right.} \bar{I}_{12 ; 34}^{\left(d, h_{1}-n_{1} / 2-n_{2}+1, n_{1}+n_{2}-1 ;-h_{2}+n_{2} / 2, \Delta+h_{2}+n_{2} / 2-1\right)}{ }_{\{F\}} F_{2}^{\prime} \cdots F_{n_{2}}^{\prime}\right) \\
& \left.-g^{E^{\prime}\left(F_{1}^{\prime}\right.} \bar{I}_{12 ; 34}^{\left(d, h_{1}-n_{1} / 2-n_{2}, n_{1}+n_{2} ;-h_{2}+n_{2} / 2, \Delta+h_{2}+n_{2} / 2-1\right)} \underset{\{F\} E}{\left.F_{2}^{\prime} \cdots F_{n_{2}}^{\prime}\right)}\right]
\end{aligned}
$$




$$
\begin{aligned}
& +\frac{2 \widetilde{K}^{\left(d, h_{2}-n_{2} / 2-2 ; \Delta+1 ; 1,0,1, n_{2}-1\right)}}{\left(n_{2}+2\right)\left(n_{2}+1\right)} \\
& \times\left[\bar{\eta}_{4 E} g^{E^{\prime}\left(F_{1}^{\prime}\right.} \bar{I}_{12 ; 34}^{\left(d, h_{1}-n_{1} / 2-n_{2}+1, n_{1}+n_{2}-1 ;-h_{2}+n_{2} / 2-1, \Delta+h_{2}+n_{2} / 2\right)}{ }_{\{F\}}^{\left.F_{2}^{\prime} \cdots F_{n_{2}}^{\prime}\right)}\right.
\end{aligned}
$$

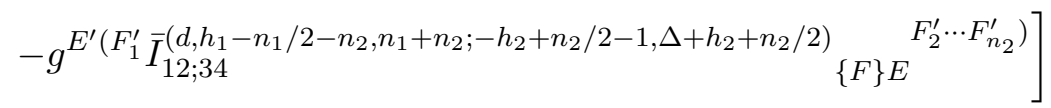

$$
\begin{aligned}
& -\left[\frac{2 \widetilde{K}^{\left(d, h_{2}-n_{2} / 2-1 ; \Delta+1 ; 1,0,0, n_{2}-1\right)}}{n_{2}+1}-\frac{2 n_{2} \widetilde{K}^{\left(d, h_{2}-n_{2} / 2-2 ; \Delta+1 ; 1,0,0, n_{2}\right)}}{\left(n_{2}+2\right)\left(n_{2}+1\right)}\right] \\
& \times\left[g_{E}{ }^{\left(F_{1}^{\prime}\right.} \bar{I}_{12 ; 34}^{\left(d, h_{1}-n_{1} / 2-n_{2}, n_{1}+n_{2} ;-h_{2}+n_{2} / 2, \Delta+h_{2}+n_{2} / 2\right)} \underset{\{F\}}{\left.F_{2}^{\prime} \cdots F_{n_{2}}^{\prime}\right) E^{\prime}}\right.
\end{aligned}
$$

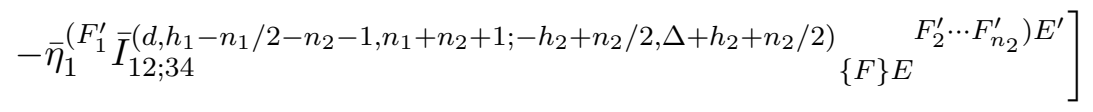

$$
\begin{aligned}
& +\frac{8 \widetilde{K}^{\left(d, h_{2}-n_{2} / 2-2 ; \Delta+1 ; 2,0,0, n_{2}-2\right)}}{\left(n_{2}+2\right)\left(n_{2}+1\right)} \\
& \times\left[g^{E^{\prime}\left(F_{1}^{\prime}\right.} g_{E} F_{2}^{\prime} \bar{I}_{12 ; 34}^{\left(d, h_{1}-n_{1} / 2-n_{2}+2, n_{1}+n_{2}-2 ;-h_{2}+n_{2} / 2-1, \Delta+h_{2}+n_{2} / 2-1\right)} \underset{\{F\}}{\left.F_{3}^{\prime} \cdots F_{n_{2}}^{\prime}\right)}\right.
\end{aligned}
$$

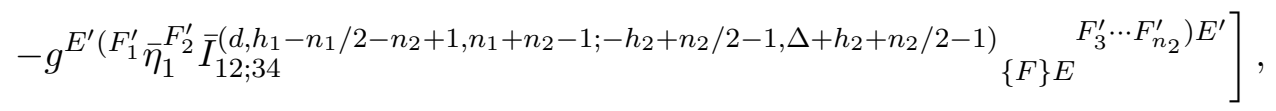

for vector exchange. Here, we first used the contiguous relations (3.9) and afterwards performed the substitutions to the four-point homogeneized embedding space coordinates (3.10). Finally, we implemented the conformal substitution (3.13) to get the preconformal blocks, after taking into account the possible simplifications stemming from contraction with the tensor structure ${ }_{b} t_{k l m}^{34}$, due to its double-transversality and tracelessness.

The corresponding results for $\ell \boldsymbol{e}_{1}$ with larger $\ell$ are obtained in a similar manner, although they become quite complicated to display due to the proliferation of indices. The complexity of the pre-conformal blocks stems from their universality: they generate all the corresponding conformal blocks once they are contracted with the appropriate tensor structures.

\subsection{2 $\ell e_{1}+e_{2}$ exchange}

For the exchange of quasi-primary operators in the $\ell \boldsymbol{e}_{1}+\boldsymbol{e}_{2}$ representation, the projection operator is simply [119]

$$
\begin{aligned}
& \left(\hat{\mathcal{P}}^{\ell \boldsymbol{e}_{1}+\boldsymbol{e}_{2}}\right)_{\nu_{2} \nu_{1} \mu_{\ell} \cdots \mu_{1}} \mu_{1}^{\prime} \cdots \mu_{\ell}^{\prime} \nu_{1}^{\prime} \nu_{2}^{\prime} \\
& \left.=\sum_{i=0}^{\lfloor\ell / 2\rfloor} a_{i} g_{\left[\nu_{1}\right.}^{\nu_{1}^{\prime}} g_{\left.\nu_{2}\right]}^{\nu_{2}^{\prime}} g_{\left(\mu_{1} \mu_{2}\right.} g^{\left(\mu_{1}^{\prime} \mu_{2}^{\prime}\right.} \cdots g_{\mu_{2 i-1} \mu_{2 i}} g^{\mu_{2 i-1}^{\prime} \mu_{2 i}^{\prime}} g_{\mu_{2 i+1}}^{\mu_{2 i+1}^{\prime}} \cdots g_{\left.\mu_{\ell}\right)} \mu_{\ell}^{\prime}\right) \\
& \left.+\sum_{i=0}^{\lfloor(\ell-1) / 2\rfloor} b_{i} g_{\left[\nu_{1}\right.}{ }^{\left[\nu_{1}^{\prime}\right.} g_{\left.\nu_{2}\right]}{ }^{\left(\mu_{1}^{\prime}\right.} g_{\left(\mu_{1}\right.}^{\left.{ }^{\prime}\right]} g_{\mu_{2} \mu_{3}} g^{\mu_{2}^{\prime} \mu_{3}^{\prime}} \cdots g_{\mu_{2 i} \mu_{2 i+1}} g^{\mu_{2 i}^{\prime} \mu_{2 i+1}^{\prime}} g_{\mu_{2 i+2}} \mu_{2 i+2}^{\prime} \cdots g_{\left.\mu_{\ell}\right)} \mu_{\ell}^{\prime}\right) \\
& \left.+\sum_{i=0}^{\lfloor(\ell-1) / 2\rfloor} c_{i} g_{\left[\nu_{1}\right.}{ }^{\left[\nu_{1}^{\prime}\right.} g_{\left.\nu_{2}\right]\left(\mu_{1}\right.} g^{\left.\nu_{2}^{\prime}\right]\left(\mu_{1}^{\prime}\right.} g_{\mu_{2} \mu_{3}} g^{\mu_{2}^{\prime} \mu_{3}^{\prime}} \cdots g_{\mu_{2 i} \mu_{2 i+1}} g^{\mu_{2 i}^{\prime} \mu_{2 i+1}^{\prime}} g_{\mu_{2 i+2}}^{\mu_{2 i+2}^{\prime}} \cdots g_{\left.\mu_{\ell}\right)}{ }^{\prime \prime}\right)
\end{aligned}
$$




$$
\begin{aligned}
& \left.+\sum_{i=0}^{\lfloor(\ell-2) / 2\rfloor} d_{i} g_{\left[\nu _ { 1 } \left(\mu_{1}\right.\right.} g^{\left[\nu _ { 1 } ^ { \prime } \left(\mu_{1}^{\prime}\right.\right.} g_{\left.\nu_{2}\right]}^{\mu_{2}^{\prime}} g_{\mu_{2}}^{\left.\nu_{2}^{\prime}\right]} g_{\mu_{3} \mu_{4}} g^{\mu_{3}^{\prime} \mu_{4}^{\prime}} \cdots g_{\mu_{2 i+1} \mu_{2 i+2}} g^{\mu_{2 i+1}^{\prime} \mu_{2 i+2}^{\prime}} g_{\mu_{2 i+3}} \mu_{2 i+3}^{\prime} \cdots g_{\left.\mu_{\ell}\right)} \mu_{\ell}^{\prime}\right) \\
& +\sum_{i=0}^{\lfloor(\ell-2) / 2\rfloor} e_{i}\left(g_{\left[\nu _ { 1 } \left(\mu_{1}\right.\right.} g_{\left.\nu_{2}\right]}^{\left[\nu_{1}^{\prime}\right.} g_{\mu_{2}}^{\left.\nu_{2}^{\prime}\right]} g^{\left(\mu_{1}^{\prime} \mu_{2}^{\prime}\right.}+g^{\left[\nu _ { 1 } ^ { \prime } \left(\mu_{1}^{\prime}\right.\right.} g_{\left[\nu_{1}\right.}^{\left.\nu_{2}^{\prime}\right]} g_{\left.\nu_{2}\right]}^{\mu_{2}^{\prime}} g_{\left(\mu_{1} \mu_{2}\right.}\right) \\
& \times g_{\mu_{3} \mu_{4}} g^{\mu_{3}^{\prime} \mu_{4}^{\prime}} \cdots g_{\mu_{2 i+1} \mu_{2 i+2}} g^{\mu_{2 i+1}^{\prime} \mu_{2 i+2}^{\prime}} g_{\mu_{2 i+3}}^{\mu_{2 i+3}^{\prime}} \cdots g_{\left.\mu_{\ell}\right)}^{\left.\mu_{\ell}^{\prime}\right)}
\end{aligned}
$$

with

$$
\begin{aligned}
a_{i} & =\frac{2}{\ell+2} \frac{(-\ell)_{2 i}}{2^{2 i} i !(-\ell+2-(d / 2+1))_{i}}, \\
c_{i} & =-\frac{(\ell-2 i)[(2 i+3) d+2(i+2) \ell-4(i+1)]}{(d+\ell-2)(d+2 \ell-2 i-2)} a_{i}, \\
b_{i} & =(\ell-2 i) a_{i}, \quad d_{i}=\frac{2(i+1)(d+2 \ell)}{d+\ell-2} a_{i+1}, \quad e_{i}=-2(i+1) a_{i+1} .
\end{aligned}
$$

It is straightforward to compute the corresponding pre-conformal blocks from the substitution rules (3.5) and (3.13). However, as the number of free indices is already large for $\ell=0$ (four free indices in total), the final result is cumbersome and not necessarily enlightening by itself. We therefore do not display it directly here, although we did use it to compare with the conformal blocks for $\ell=0$ and $\ell=1$ obtained later.

We would like to note that, apart from the prefactor $2 /(\ell+2)$, the coefficients $a_{i}$ in (4.5) are identical to those appearing in the hatted projection operator for $\ell \boldsymbol{e}_{1}$ (4.1) with $d \rightarrow d+2$. This observation, which comes about from the equivalent role played by $\ell$ in all towers of irreducible representations $\boldsymbol{N}_{m}=\boldsymbol{N}+\ell \boldsymbol{e}_{1}$, will have far-reaching consequences later on.

\subsection{Conformal blocks and four-point correlation functions}

On the one hand, the conformal blocks can be obtained directly from the pre-conformal blocks. On the other, they can be computed in two steps, exploiting the contiguous relations to simplify the contraction with the tensor structures after the first conformal substitution. In both cases, the final result is the same, although it is more efficient to use the contiguous relations to simplify the conformal blocks. The convenience of the pre-conformal blocks is that they are fully determined as soon as the irreducible representation of the exchanged quasi-primary operator is known.

Here we have computed the conformal blocks for four four-point correlation functions: symmetric-traceless exchange in scalar-scalar-scalar-scalar, symmetric-traceless exchange in scalar-scalar-scalar- $\boldsymbol{e}_{2}$, symmetric-traceless exchange and $\boldsymbol{\ell} \boldsymbol{e}_{1}+\boldsymbol{e}_{2}$ exchange in scalarvector-scalar-vector, and symmetric-traceless exchange in scalar-scalar-vector-vector. In all cases, all the possible exchanged quasi-primary operators are considered, and the conformal blocks in all OPE channels are obtained, allowing the implementation of the conformal bootstrap. The first, third and fourth four-point correlation functions are chosen for comparison with the literature, while the second set of conformal blocks is a proof-of-concept example, which shows that we are able to compute any conformal block, albeit in a sim- 
ple example with only one tower of exchanged quasi-primary operators with one tensor structure each. $^{4}$

The conformal blocks $\mathscr{G}_{(a \mid b)}^{i j|m| k l}(3.15)$ are naturally obtained in the OPE tensor structure basis. Indeed, the tensor structures used to compute the conformal blocks are the ones appearing in the OPE. However, since three-point correlation functions appear directly in (3.15), it is possible to obtain the conformal blocks $\mathscr{G}_{[a \mid b]}^{i j|m| k l}$ in the three-point function tensor structure basis. Obviously, the conformal blocks obtained from the OPE tensor structures are linear combinations of those obtained from the three-point function tensor structures. Therefore, the conformal blocks in the latter basis are obtained from the former ones with the help of (invertible) transformation matrices $R_{i j m}$ and $R_{k l m}$ as

$$
\mathscr{G}_{[a \mid b]}^{i j|m| k l}=\left(R_{i j m}\right)_{a a^{\prime}}\left(R_{k l m}\right)_{b b^{\prime}} \mathscr{G}_{\left(a^{\prime} \mid b^{\prime}\right)}^{i j|m| k l}
$$

The distinction is irrelevant when there is just a single conformal block (the transformation matrices are simply multiplicative factors), but in cases with more than one block, the difference is important. We will see later that the best way of representing conformal blocks originates from a mixed basis of tensor structures,

$$
\mathscr{G}_{(a \mid b]}^{i j|m| k l}=\left(R_{k l m}\right)_{b b^{\prime}} \mathscr{G}_{\left(a \mid b^{\prime}\right)}^{i j|m| k l},
$$

where ${ }_{b} t_{k \ell m}^{34}$ are natural three-point function tensor structures, while ${ }_{a} t_{i j}^{12 m}$ are natural OPE tensor structures. The examples below will clarify this distinction.

To simplify the notation, in the following, conformal blocks will be denoted by $\mathscr{G}_{(a \mid b)}^{N}$, $\mathscr{G}_{[a \mid b]}^{N}$ or $\mathscr{G}_{(a \mid b]}^{N}$ for an exchanged quasi-primary operator in the irreducible representation $\boldsymbol{N}$ with the OPE or three-point function tensor structures $a$ and $b$, irrespective of the four-point correlation function under consideration.

\subsubsection{Symmetric-traceless exchange in scalar-scalar-scalar-scalar}

For our first example, we focus on the classic case of symmetric-traceless exchange in the four-point correlation function of four scalars. It is straightforward to compute the conformal blocks (3.15) from the pre-conformal blocks (4.3) and (4.4). Here we have only one tensor structure of each type; hence, the indices $a$ and $b$ are superfluous.

For scalar exchange, the normalization constant and tensor structures are simply $\lambda_{\mathbf{0}}=$ ${ }_{1} t_{i j}^{12 m}={ }_{1} t_{k l m}^{34}=1$. These result in

$$
\begin{aligned}
\mathscr{G}_{(1 \mid 1)}^{\mathbf{0}}= & \rho^{\left(d, h_{k l m} ; \Delta_{m}\right)} \bar{I}_{12 ; 34}^{\left(d, h_{i j m}, 0 ;-h_{k l m}, \Delta_{m}+h_{k l m}\right)} \\
= & \rho^{\left(d, h_{i j m} ; \Delta_{m}\right)} \rho^{\left(d, h_{k l m} ; \Delta_{m}\right)} x_{3}^{\Delta_{m}+h_{i j m}} K_{12 ; 34 ; 3}^{\left(d, h_{i j m} ;-h_{k l m}, \Delta_{m}+h_{k l m}\right)}\left(x_{3} ; y_{4}\right) \\
= & \rho^{\left(d, h_{i j m} ; \Delta_{m}\right)} \rho^{\left(d, h_{k l m} ; \Delta_{m}\right)}\left(\frac{u}{v}\right)^{\Delta_{m}+h_{i j m}} \\
& \times G\left(\Delta_{m}+h_{k l m}, \Delta_{m}+h_{i j m}, \Delta_{m}+1-d / 2, \Delta_{m} ; u / v, 1-1 / v\right),
\end{aligned}
$$

\footnotetext{
${ }^{4}$ The number of conformal blocks increases quite quickly for generic four-point correlation functions. For example, $\boldsymbol{e}_{1}+\boldsymbol{e}_{2}$ exchange in spinor- $\left(\boldsymbol{e}_{1}+\boldsymbol{e}_{r}\right)$-scalar- $\boldsymbol{e}_{2}$ already has 24 different blocks. Such a large number of conformal blocks is not convenient for the format of a typical article.
} 
while for vector exchange, the normalization constant is $\lambda_{\boldsymbol{e}_{1}}=1 / \sqrt{d}$, and the tensor structures are $\left({ }_{1} t_{i j}^{12 m}\right)^{E F}=\mathcal{A}_{12}^{E F} / \sqrt{d}$ and $\left({ }_{1} t_{k l m}^{34}\right)_{E^{\prime} F^{\prime}}=\mathcal{A}_{34 E^{\prime} F^{\prime}} / \sqrt{d}$, giving

$$
\begin{aligned}
\mathscr{G}_{(1 \mid 1)}^{\boldsymbol{e}_{1}}= & \frac{(-2)^{h_{k l m}+1 / 2}\left(h_{k l m}+1 / 2\right)\left(d-1-\Delta_{m}\right)}{d^{3 / 2}}\left(\Delta_{m}+1\right)_{h_{k l m}-1 / 2}\left(\Delta_{m}+1-d / 2\right)_{h_{k l m}-1 / 2} \\
& \times\left[\frac{1}{x_{4}} \bar{I}_{12 ; 34}^{\left(d, h_{i j m}+1 / 2,0 ;-h_{k l m}-1 / 2, \Delta_{m}+h_{k l m}+1 / 2\right)}\right. \\
& +\frac{\left(2 h_{i j m}+1\right)\left(2 h_{i j m}-1+d\right)}{2} \bar{I}_{12 ; 34}^{\left(d, h_{i j m}-1 / 2,0 ;-h_{k l m}-1 / 2, \Delta_{m}+h_{k l m}+1 / 2\right)} \\
& -\frac{1}{x_{3}} \bar{I}_{12 ; 34}^{\left(d, h_{i j m}+1 / 2,0 ;-h_{k l m}+1 / 2, \Delta_{m}+h_{k l m}-1 / 2\right)} \\
& \left.+\frac{\left(2 h_{i j m}+1\right)\left(2 h_{i j m}-1+d\right)}{2} \bar{I}_{12 ; 34}^{\left(d, h_{i j m}-1 / 2,0 ;-h_{k l m}+1 / 2, \Delta_{m}+h_{k l m}-1 / 2\right)}\right] .
\end{aligned}
$$

Up to a different normalization, these results match with the usual ones found in the literature [104].

The other conformal blocks for the $\ell \boldsymbol{e}_{1}$ irreducible representations can be obtained in the same manner, although it is simpler to rely on the contiguous relations after the first conformal substitution. Indeed, from the three-point correlation functions [174]

$$
\begin{aligned}
\lambda_{\ell e_{1}}\left(\overline{\bar{J}}_{34 ; 2}^{\left(d, h_{k l m}, \ell, \Delta_{m}, \ell e_{1}\right)} \cdot{ }_{1} t_{k l m}^{34}\right)_{E_{\ell}^{\prime \prime} \cdots E_{1}^{\prime \prime}}= & \frac{(-2)^{h_{k l m}-\ell / 2} 2^{\ell} \ell !\left(h_{k l m}-\ell / 2+1\right)_{\ell}\left(d-1-\Delta_{m}\right)_{\ell}}{(d+2 \ell-2)(d-1)_{\ell-1}} \\
& \times\left(\Delta_{m}+\ell\right)_{h_{k l m}-\ell / 2}\left(\Delta_{m}+1-d / 2\right)_{h_{k l m}-\ell / 2} \overline{\bar{\eta}}_{4 E_{\ell}^{\prime \prime}} \cdots \overline{\bar{\eta}}_{4 E_{1}^{\prime \prime}},
\end{aligned}
$$

the tensor structure, see (4.1),

$$
\begin{gathered}
\left({ }_{1} t_{i j}^{12 m}\right)^{E_{1} \cdots E_{\ell} F_{1} \cdots F_{\ell}}=\lambda_{\ell e_{1}} \sum_{i=0}^{\lfloor\ell / 2\rfloor} \frac{(-\ell)_{2 i}}{2^{2 i} i !(-\ell+2-d / 2)_{i}} \mathcal{A}_{12}^{\left(E_{1} E_{2}\right.} \mathcal{A}_{12}^{\left(F_{1} F_{2}\right.} \cdots \mathcal{A}_{12}^{E_{2 i-1} E_{2 i}} \mathcal{A}_{12}^{F_{2 i-1} F_{2 i}} \\
\times \mathcal{A}_{12}^{E_{2 i+1} F_{2 i+1}} \cdots \mathcal{A}_{12}^{\left.\left.E_{\ell}\right) F_{\ell}\right)},
\end{gathered}
$$

and the normalization constant $\lambda_{\ell e_{1}}=\sqrt{\ell ! /\left[(d+2 \ell-2)(d-1)_{\ell-1}\right]}$, the conformal blocks are given by (with $n_{a}=\ell$ )

$$
\begin{aligned}
\mathscr{G}_{(1 \mid 1)}^{\ell e_{1}}= & \frac{(-2)^{h_{k l m}-\ell / 2} 2^{\ell} \ell !\left(h_{k l m}-\ell / 2+1\right)_{\ell}\left(d-1-\Delta_{m}\right)_{\ell}}{(d+2 \ell-2)(d-1)_{\ell-1}}\left(\Delta_{m}+\ell\right)_{h_{k l m}-\ell / 2}\left(\Delta_{m}+1-d / 2\right)_{h_{k l m}-\ell / 2} \\
& \times\left({ }_{1} t_{i j}^{12 m}\right)^{E_{1} \cdots E_{\ell} F_{1} \cdots F_{\ell}}\left(x_{3}^{-\ell / 2} x_{4}^{\ell / 2}\left(\hat{\mathcal{P}}_{21}^{\ell e_{1}} \cdot \hat{\mathcal{P}}_{23}^{\ell e_{1}}\right)_{\{E\}}{ }^{\left\{E^{\prime \prime}\right\}_{\eta}} \bar{\eta}_{4 E_{\ell}^{\prime \prime}} \cdots \bar{\eta}_{4 E_{1}^{\prime \prime}}\right)_{c s_{4}} \\
= & \frac{(-2)^{h_{k l m}-\ell / 2} 2^{\ell} \ell !\left(h_{k l m}-\ell / 2+1\right)_{\ell}\left(d-1-\Delta_{m}\right)_{\ell}}{(d+2 \ell-2)(d-1)_{\ell-1}}\left(\Delta_{m}+\ell\right)_{h_{k l m}-\ell / 2}\left(\Delta_{m}+1-d / 2\right)_{h_{k l m}-\ell / 2} \\
& \times\left({ }_{1} t_{i j}^{12 m}\right)^{E_{1} \cdots E_{\ell} F_{1} \cdots F_{\ell}} \sum_{i=0}^{\ell \ell / 2\rfloor} \frac{(-\ell)_{2 i}}{2^{2 i} i !(-\ell+2-d / 2)_{i}}\left(x_{3}^{-\ell / 2} x_{4}^{\ell / 2}\left(\bar{\eta}_{4} \cdot \mathcal{A}_{23} \cdot \bar{\eta}_{4}\right)^{i}\right. \\
& \left.\times \mathcal{A}_{12\left(E_{1} E_{2}\right.} \cdots \mathcal{A}_{12 E_{2 i-1} E_{2 i}}\left(\mathcal{A}_{123} \cdot \bar{\eta}_{4}\right)_{E_{2 i+1}} \cdots\left(\mathcal{A}_{123} \cdot \bar{\eta}_{4}\right)_{\left.E_{\ell}\right)}\right)_{c s_{4}} .
\end{aligned}
$$


Since the metrics $g_{E_{i} E_{j}}$ and the embedding space coordinates $\bar{\eta}_{1 E_{i}}$ commute with the conformal substitution and vanish once contracted with the tensor structure, only the $i=0$ term survives in (4.9). The expression then simplifies to

$$
\begin{aligned}
\mathscr{G}_{(1 \mid 1)}^{\ell e_{1}}= & \frac{(-2)^{h_{k l m}-\ell / 2} 2^{\ell} \ell !\left(h_{k l m}-\ell / 2+1\right)_{\ell}\left(d-1-\Delta_{m}\right)_{\ell}}{(d+2 \ell-2)(d-1)_{\ell-1}}\left(\Delta_{m}+\ell\right)_{h_{k l m}-\ell / 2}\left(\Delta_{m}+1-d / 2\right)_{h_{k l m}-\ell / 2} \\
& \times\left({ }_{1} t_{i j}^{12 m}\right)^{E_{1} \cdots E_{\ell} F_{1} \cdots F_{\ell}}\left(x_{3}^{-\ell / 2} x_{4}^{\ell / 2}\left(\mathcal{A}_{123} \cdot \bar{\eta}_{4}\right)_{E_{1}} \cdots\left(\mathcal{A}_{123} \cdot \bar{\eta}_{4}\right)_{E_{\ell}}\right)_{c s_{4}} \\
= & \frac{(-2)^{h_{k l m}-\ell / 2} 2^{\ell} \ell !\left(h_{k l m}-\ell / 2+1\right)_{\ell}\left(d-1-\Delta_{m}\right)_{\ell}}{(d+2 \ell-2)(d-1)_{\ell-1}}\left(\Delta_{m}+\ell\right)_{h_{k l m}-\ell / 2}\left(\Delta_{m}+1-d / 2\right)_{h_{k l m}-\ell / 2} \\
& \times \lambda_{\ell e_{1}} \sum_{i=0}^{\lfloor\ell / 2\rfloor} \frac{(-2)^{i}(-\ell)_{2 i}}{2^{2 i} i !(-\ell+2-d / 2)_{i}} g^{E_{1} E_{2}} \bar{\eta}_{1}^{F_{1}} \bar{\eta}_{2}^{F_{2}} \cdots g^{E_{2 i-1} E_{2 i}} \bar{\eta}_{1}^{F_{2 i-1}} \bar{\eta}_{2}^{F_{2 i}} \\
& \times \mathcal{A}_{12}^{E_{2 i+1} F_{2 i+1}} \cdots \mathcal{A}_{12}^{E_{\ell} F_{\ell}}\left(x_{3}^{-\ell / 2} x_{4}^{\ell / 2}\left(\mathcal{A}_{123} \cdot \bar{\eta}_{4}\right)_{E_{1}} \cdots\left(\mathcal{A}_{123} \cdot \bar{\eta}_{4}\right)_{E_{\ell}}\right)_{c s_{4}} .
\end{aligned}
$$

In the last equality above, we removed the explicit symmetrizations over the sets of $\{F\}$ and $\{E\}$ due to the symmetry properties of the $\bar{I}$-functions and the product of $\mathcal{A}_{123} \cdot \bar{\eta}_{4}$, respectively. We also used the fact that only the metrics $g^{E_{i} E_{j}}$ in the trace terms do not vanish when contracted.

Moreover, the contiguous relations (3.20) were used to transform $\mathcal{A}_{12}^{F_{i} F_{j}}$ into $-2 \bar{\eta}_{1}^{F_{i}} \bar{\eta}_{2}^{F_{j}}$. Contracting the embedding space metrics and using simple relations for the product of $\mathcal{A}$-metrics, we obtain

$$
\begin{aligned}
\mathscr{G}_{(1 \mid 1)}^{\ell e_{1}}= & \frac{(-2)^{h_{k l m}-\ell / 2} 2^{\ell} \ell !\left(h_{k l m}-\ell / 2+1\right)_{\ell}\left(d-1-\Delta_{m}\right)_{\ell}}{(d+2 \ell-2)(d-1)_{\ell-1}}\left(\Delta_{m}+\ell\right)_{h_{k l m}-\ell / 2}\left(\Delta_{m}+1-d / 2\right)_{h_{k l m}-\ell / 2} \\
& \times \lambda_{\ell e_{1}} \sum_{i=0}^{\lfloor\ell / 2\rfloor} \frac{(-2)^{i}(-\ell)_{2 i}}{2^{2 i} i !(-\ell+2-d / 2)_{i}} \bar{\eta}_{1}^{F_{1}} \bar{\eta}_{2}^{F_{2}} \cdots \bar{\eta}_{1}^{F_{2 i-1}} \bar{\eta}_{2}^{F_{2 i}} \mathcal{A}_{12}^{E_{2 i+1} F_{2 i+1}} \cdots \mathcal{A}_{12}^{E_{\ell} F_{\ell}} \\
& \times\left(x_{3}^{-\ell / 2} x_{4}^{\ell / 2}\left(\bar{\eta}_{4} \cdot \mathcal{A}_{23} \cdot \bar{\eta}_{4}\right)^{i}\left(\mathcal{A}_{123} \cdot \bar{\eta}_{4}\right)_{E_{2 i+1}} \cdots\left(\mathcal{A}_{123} \cdot \bar{\eta}_{4}\right)_{E_{\ell}}\right)_{c s_{4}} \\
= & \frac{(-2)^{h_{k l m}-\ell / 2} 2^{\ell} \ell !\left(h_{k l m}-\ell / 2+1\right)_{\ell}\left(d-1-\Delta_{m}\right)_{\ell}}{(d+2 \ell-2)(d-1)_{\ell-1}}\left(\Delta_{m}+\ell\right)_{h_{k l m}-\ell / 2}\left(\Delta_{m}+1-d / 2\right)_{h_{k l m}-\ell / 2} \\
& \times \lambda_{\ell e_{1}} \sum_{i=0}^{\lfloor\ell / 2\rfloor} \frac{(-\ell)_{2 i}}{i !(-\ell+2-d / 2)_{i}} \bar{\eta}_{1}^{F_{1}} \bar{\eta}_{2}^{F_{2}} \cdots \bar{\eta}_{1}^{F_{2 i-1}} \bar{\eta}_{2}^{F_{2 i}} \mathcal{A}_{12}^{E_{2 i+1} F_{2 i+1}} \cdots \mathcal{A}_{12}^{E_{\ell} F_{\ell}} \\
& \times\left(x_{3}^{-\ell / 2+i} x_{4}^{-\ell / 2+i}\left[x_{4}\left(\bar{\eta}_{4}-\bar{\eta}_{2}\right)-x_{3}\left(\bar{\eta}_{3}-\bar{\eta}_{2}\right)\right]_{E_{2 i+1}} \cdots\left[x_{4}\left(\bar{\eta}_{4}-\bar{\eta}_{2}\right)-x_{3}\left(\bar{\eta}_{3}-\bar{\eta}_{2}\right)\right]_{E_{\ell}}\right)_{c s_{4}} .
\end{aligned}
$$

From the contiguous relations (3.20), it is clear that the metrics $g^{E_{i} F_{i}}$ lead to vanishing contributions. Indeed, if the conformal substitution is performed on terms containing $\bar{\eta}_{2 E_{j}}$, they lead to traces which vanish identically. Moreover, if the conformal substitution is done on terms with $\left(x_{4} \bar{\eta}_{4}-x_{3} \bar{\eta}_{3}\right)_{E_{j}}$, the two contributions cancel due to the contiguous relations (3.20). Thus, in (4.11) one can replace $\mathcal{A}_{12}^{E_{i} F_{i}}$ by $-\bar{\eta}_{1}^{E_{i}} \bar{\eta}_{2}^{F_{i}}-\bar{\eta}_{2}^{E_{i}} \bar{\eta}_{1}^{F_{i}}$. However, the 
contractions with $-\bar{\eta}_{1}^{E_{i}} \bar{\eta}_{2}^{F_{i}}$ vanish identically, leading to

$$
\begin{aligned}
\mathscr{G}_{(1 \mid 1)}^{\ell e_{1}}= & \frac{(-2)^{h_{k l m}-\ell / 2} 2^{\ell} \ell !\left(h_{k l m}-\ell / 2+1\right)_{\ell}\left(d-1-\Delta_{m}\right)_{\ell}}{(d+2 \ell-2)(d-1)_{\ell-1}}\left(\Delta_{m}+\ell\right)_{h_{k l m}-\ell / 2}\left(\Delta_{m}+1-d / 2\right)_{h_{k l m}-\ell / 2} \\
& \times \lambda_{\ell e_{1}} \sum_{i=0}^{\lfloor\ell / 2\rfloor} \frac{(-1)^{\ell}(-\ell)_{2 i}}{i !(-\ell+2-d / 2)_{i}} \bar{\eta}_{1}^{F_{1}} \bar{\eta}_{2}^{F_{2}} \cdots \bar{\eta}_{1}^{F_{2 i-1}} \bar{\eta}_{2}^{F_{2 i}} \bar{\eta}_{2}^{E_{2 i+1}} \bar{\eta}_{1}^{F_{2 i+1}} \cdots \bar{\eta}_{2}^{E_{\ell}} \bar{\eta}_{1}^{F_{\ell}} \\
& \times\left(x_{3}^{-\ell / 2+i} x_{4}^{-\ell / 2+i}\left[x_{4}\left(\bar{\eta}_{4}-\bar{\eta}_{2}\right)-x_{3}\left(\bar{\eta}_{3}-\bar{\eta}_{2}\right)\right]_{E_{2 i+1}} \cdots\left[x_{4}\left(\bar{\eta}_{4}-\bar{\eta}_{2}\right)-x_{3}\left(\bar{\eta}_{3}-\bar{\eta}_{2}\right)\right]_{E_{\ell}}\right)_{c s_{4}} .
\end{aligned}
$$

At this point, we only need to proceed with the conformal substitution (3.13) and the contiguous relations (3.20). Moreover, the contractions are straightforward since all the $E$-indices are symmetrized and the $\bar{I}$-functions are totally symmetrized. Hence, the indices can be forgotten and (4.12) can be rewritten efficiently as

$$
\begin{aligned}
\mathscr{G}_{(1 \mid 1)}^{\ell e_{1}}= & \frac{(-2)^{h_{k l m}+\ell / 2} 2^{\ell} \ell !\left(h_{k l m}-\ell / 2+1\right)_{\ell}\left(d-1-\Delta_{m}\right)_{\ell}}{(d+2 \ell-2)(d-1)_{\ell-1}}\left(\Delta_{m}+\ell\right)_{h_{k l m}-\ell / 2}\left(\Delta_{m}+1-d / 2\right)_{h_{k l m}-\ell / 2} \\
& \times \lambda_{\ell e_{1}}\left(\sum_{n=0}^{\lfloor\ell / 2\rfloor} \frac{(-\ell)_{2 n}}{2^{2 n} n !(-\ell+2-d / 2)_{n}}\left[\frac{\left(\alpha_{4}-\alpha_{2}\right) x_{4}-\left(\alpha_{3}-\alpha_{2}\right) x_{3}}{2}\right]^{\ell-2 n}\right)_{s} \\
= & \omega\left(h_{k l m}, \Delta_{m}, \ell\right)\left(C_{\ell}^{(d / 2-1)}(X)\right)_{s}
\end{aligned}
$$

where the normalization constant is

$$
\begin{aligned}
\omega\left(h_{k l m}, \Delta_{m}, \ell\right)= & \frac{(-2)^{h_{k l m}+\ell / 2} 2^{\ell} \ell !\left(h_{k l m}-\ell / 2+1\right)_{\ell}\left(d-1-\Delta_{m}\right)_{\ell}}{(d+2 \ell-2)(d-1)_{\ell-1}} \\
& \times\left(\Delta_{m}+\ell\right)_{h_{k l m}-\ell / 2}\left(\Delta_{m}+1-d / 2\right)_{h_{k l m}-\ell / 2} \frac{\lambda_{\ell e_{1}} \ell !}{2^{\ell}(d / 2-1)_{\ell}},
\end{aligned}
$$

the $C_{\ell}^{(d / 2-1)}(X)$ are the usual Gegenbauer polynomials in terms of the variable

$$
X=\frac{\left(\alpha_{4}-\alpha_{2}\right) x_{4}-\left(\alpha_{3}-\alpha_{2}\right) x_{3}}{2}
$$

and the $s$-substitution is

$$
\begin{aligned}
s: & \alpha_{2}^{s_{2}} \alpha_{3}^{s_{3}} \alpha_{4}^{s_{4}} x_{3}^{r_{3}} x_{4}^{r_{4}} \rightarrow G_{(0,0,0,0,0)}^{i j|m| k l} \\
= & \rho^{\left(d,\left(\ell+s_{2}-s_{3}-s_{4}\right) / 2 ;-h_{i j m}-\ell / 2\right)} x_{3}^{-s_{3}} x_{4}^{-s_{4}} \\
& \times \bar{I}_{12 ; 34}^{\left(d, h_{i j m}-\left(s_{2}-s_{3}-s_{4}\right) / 2,0 ;-h_{k l m}+\left(r_{3}-r_{4}\right) / 2, \Delta_{m}+h_{k l m}-\left(r_{3}-r_{4}\right) / 2\right)} \\
= & \rho^{\left(d,\left(\ell+s_{2}-s_{3}-s_{4}\right) / 2 ;-h_{i j m}-\ell / 2\right)} \rho^{\left(d, h_{i j m}-\left(s_{2}-s_{3}-s_{4}\right) / 2 ; \Delta_{m}\right)} x_{3}^{\Delta_{m}+h_{i j m}-\left(s_{2}+s_{3}-s_{4}\right) / 2} x_{4}^{-s_{4}} \\
& \times K_{12 ; 34 ; 3}^{\left(d, h_{i j m}-\left(s_{2}-s_{3}-s_{4}\right) / 2 ;-h_{k l m}+\left(r_{3}-r_{4}\right) / 2, \Delta_{m}+h_{k l m}-\left(r_{3}-r_{4}\right) / 2\right)}\left(x_{3} ; y_{4}\right) \\
= & \rho^{\left(d,\left(\ell+s_{2}-s_{3}-s_{4}\right) / 2 ;-h_{i j m}-\ell / 2\right)} \rho^{\left(d, h_{i j m}-\left(s_{2}-s_{3}-s_{4}\right) / 2 ; \Delta_{m}\right)}(u / v)^{\Delta_{m}+h_{i j m}-\left(s_{2}+s_{3}-s_{4}\right) / 2} u^{-s_{4}} \\
& \times G\left(\Delta_{m}+h_{k l m}-\left(r_{3}-r_{4}\right) / 2, \Delta_{m}+h_{i j m}-\left(s_{2}-s_{3}-s_{4}\right) / 2, \Delta_{m}+1-d / 2, \Delta_{m} ; u / v ; 1-1 / v\right) .
\end{aligned}
$$


Here, the $\alpha_{i}$ are placeholders for the $s$-substitution that enable a very convenient form for the conformal blocks. Indeed, (4.13) gives all the exchanged conformal blocks once the simple $s$-substitution is performed. The latter is straightforwardly determined by first contracting the $\bar{\eta}_{2}^{E_{i}}$ with the $\bar{\eta}_{3 E_{i}}$ and $\bar{\eta}_{4 E_{i}}$, followed by the usual conformal substitution with the contiguous relations for the $\bar{\eta}_{1}^{F_{i}}$ and the remaining $\bar{\eta}_{2}^{E_{i}}$ and $\bar{\eta}_{2}^{F_{i}}$. Finally, the explicit dependence on the dummy summation index $n$ is transformed into a dependence on $\ell$ and $s_{i}$ or $r_{i}$ so that the final substitution can be pulled outside of the sum. The presence of $\ell$, $s_{i}$ and $r_{i}$ in (3.21) should now be clear. The explicit form (4.13) in terms of Gegenbauer polynomials with proper substitutions is natural from the $\ell \boldsymbol{e}_{1}$ projection operator and it is an interesting feature that generalizes to all conformal blocks. Moreover, it allows for a very effective way of determining conformal blocks for larger $\ell$.

Although (4.13) is our final result, we can obtain more explicit equations for the conformal blocks that can be compared with the literature. For example, using the binomial expansion for $X$, the conformal blocks (4.13) can be rewritten as [119]

$$
\begin{aligned}
\mathscr{G}_{(1 \mid 1)}^{\ell e_{1}}= & \frac{\omega\left(h_{k l m}, \Delta_{m}, \ell\right)}{\Gamma(d / 2-1)} \sum_{n_{1}=0}^{\lfloor\ell / 2\rfloor} \sum_{n_{2}=0}^{\ell-2 n_{1} \ell-2 n_{1}-n_{2}} \sum_{n_{3}=0}^{n_{2}} \sum_{n_{4}=0}^{n_{2}} \frac{(-1)^{n_{1}+n_{2}+n_{3}+n_{4}} \Gamma\left(\ell-n_{1}+d / 2-1\right)}{n_{1} ! \Gamma\left(\ell-2 n_{1}+1\right)}\left(\begin{array}{c}
\ell-2 n_{1} \\
n_{2}
\end{array}\right) \\
& \times\left(\begin{array}{c}
\ell-2 n_{1}-n_{2} \\
n_{3}
\end{array}\right)\left(\begin{array}{l}
n_{2} \\
n_{4}
\end{array}\right) \rho^{\left(d, n_{1}+n_{2} ;-h_{i j m}-\ell / 2\right)} x_{3}^{-n_{3}} x_{4}^{-\ell+2 n_{1}+n_{2}+n_{3}} \\
& \times \bar{I}_{12 ; 34}^{\left(d, h_{i j m}+\ell / 2-n_{1}-n_{2}, 0 ;-h_{k l m}-\ell / 2+n_{1}+n_{3}+n_{4}, \Delta_{m}+h_{k l m}+\ell / 2-n_{1}-n_{3}-n_{4}\right)} \\
= & \frac{\omega\left(h_{k l m}, \Delta_{m}, \ell\right)}{\Gamma(d / 2-1)} \sum_{n_{1}=0}^{\lfloor\ell / 2\rfloor} \sum_{n_{2}=0}^{\ell-2 n_{1}} \sum_{n_{3}=0}^{\ell-2 n_{1}-n_{2}} \sum_{n_{4}=0}^{n_{2}} \frac{(-1)^{n_{1}+n_{2}+n_{3}+n_{4}} \Gamma\left(\ell-n_{1}+d / 2-1\right)}{n_{1} ! \Gamma\left(\ell-2 n_{1}+1\right)}\left(\begin{array}{c}
\ell-2 n_{1} \\
n_{2}
\end{array}\right) \\
& \times\left(\begin{array}{c}
\ell-2 n_{1}-n_{2} \\
n_{3}
\end{array}\right)\left(\begin{array}{c}
n_{2} \\
n_{4}
\end{array}\right) \rho^{\left(d, n_{1}+n_{2} ;-h_{i j m}-\ell / 2\right)} x_{3}^{-n_{3}} x_{4}^{-\ell+2 n_{1}+n_{2}+n_{3}} \\
& \times\left.\frac{\mathscr{G}_{(1 \mid 1)}^{0}}{\omega\left(h_{k l m}, \Delta_{m}, 0\right)}\right|_{h_{i j m} \rightarrow h_{i j m}+\ell / 2-n_{1}-n_{2}, h_{k l m} \rightarrow h_{k l m}+\ell / 2-n_{1}-n_{3}-n_{4}}
\end{aligned}
$$

where $\mathscr{G}_{(1 \mid 1)}^{\mathbf{0}}$ is the conformal block for scalar exchange.

Here we have noted that [see (3.16)]

$$
\begin{aligned}
& \bar{I}_{12 ; 34}^{\left(d, h_{i j m}+\ell / 2-n_{1}-n_{2}, 0 ;-h_{k l m}-\ell / 2+n_{1}+n_{3}+n_{4}, \Delta_{m}+h_{k l m}+\ell / 2-n_{1}-n_{3}-n_{4}\right)} \\
= & \rho^{\left(d, h_{i j m}+\ell / 2-n_{1}-n_{2} ; \Delta_{m}\right)} x_{3}^{\Delta_{m}+h_{i j m}+\ell / 2-n_{1}-n_{2}} \\
& G\left(\Delta_{m}+h_{k l m}+\ell / 2-n_{1}-n_{3}-n_{4}, \Delta_{m}+h_{i j m}+\ell / 2-n_{1}-n_{2}, \Delta_{m}+1\right. \\
& \left.-d / 2, \Delta_{m} ; u / v, 1-1 / v\right),
\end{aligned}
$$

and that

$$
\begin{aligned}
\mathscr{G}_{(1 \mid 1)}^{\mathbf{0}}= & \omega\left(h_{k l m}, \Delta_{m}, 0\right) \rho^{\left(d, h_{i j m} ; \Delta_{m}\right)} x_{3}^{h_{i j m}+\Delta_{m}} G\left(\Delta_{m}+h_{k l m}, \Delta_{m}+h_{i j m}, \Delta_{m}+1\right. \\
& \left.-d / 2, \Delta_{m}, u / v, 1-1 / v\right),
\end{aligned}
$$


so that

$$
\begin{aligned}
& \bar{I}_{12 ; 34}^{\left(d, h_{i j m}+\ell / 2-n_{1}-n_{2}, 0 ;-h_{k l m}-\ell / 2+n_{1}+n_{3}+n_{4}, \Delta_{m}+h_{k l m}+\ell / 2-n_{1}-n_{3}-n_{4}\right)} \\
& =\left.\frac{\mathscr{G}_{(1 \mid 1)}^{\mathbf{0}}}{\omega\left(h_{k l m}, \Delta_{m}, 0\right)}\right|_{h_{i j m} \rightarrow h_{i j m}+\ell / 2-n_{1}-n_{2}, h_{k l m} \rightarrow h_{k l m}+\ell / 2-n_{1}-n_{3}-n_{4}} .
\end{aligned}
$$

From the recurrence relation for Gegenbauer polynomials, it is also easy to get the recurrence relation for the conformal blocks (4.13) as [104]

$$
\begin{aligned}
\mathscr{G}_{(1 \mid 1)}^{\ell e_{1}}= & \omega\left(h_{k l m}, \Delta_{m}, \ell\right)\left(\frac{2 \ell+d-4}{\ell} X C_{\ell-1}^{(d / 2-1)}(X)-\frac{\ell+d-4}{\ell} C_{\ell-2}^{(d / 2-1)}(X)\right)_{s} \\
= & \frac{2 \ell+d-4}{2 \ell}\left[\frac{\omega\left(h_{k l m}, \Delta_{m}, \ell\right)}{\omega\left(h_{k l m}+1 / 2, \Delta_{m}, \ell-1\right)} \frac{1}{x_{4}}\left(\mathscr{G}_{(1 \mid 1)}^{(\ell-1) \boldsymbol{e}_{1}}\right)_{h_{i j m} \rightarrow h_{i j m}+1 / 2} h_{k l m} \rightarrow h_{k l m}+1 / 2\right. \\
& +\frac{\omega\left(h_{k l m}, \Delta_{m}, \ell\right)}{\omega\left(h_{k l m}+1 / 2, \Delta_{m}, \ell-1\right)} \frac{\left(2 h_{i j m}+\ell\right)\left(2 h_{i j m}+\ell-2+d\right)}{2}\left(\mathscr{G}_{(1 \mid 1)}^{(\ell-1) e_{1}}\right)_{\substack{h_{i j m} \rightarrow h_{i j m}-1 / 2 \\
h_{k l m} \rightarrow h_{k l m}+1 / 2}} \\
& -\frac{\omega\left(h_{k l m}, \Delta_{m}, \ell\right)}{\omega\left(h_{k l m}-1 / 2, \Delta_{m}, \ell-1\right)} \frac{1}{x_{3}}\left(\mathscr{G}_{(1 \mid 1)}^{(\ell-1) e_{1}}\right)_{h_{i j m} \rightarrow h_{i j m}+1 / 2} h_{k l m} \rightarrow h_{k l m}-1 / 2 \\
& \left.-\frac{\omega\left(h_{k l m}, \Delta_{m}, \ell\right)}{\omega\left(h_{k l m}-1 / 2, \Delta_{m}, \ell-1\right)} \frac{\left(2 h_{i j m}+\ell\right)\left(2 h_{i j m}+\ell-2+d\right)}{2}\left(\mathscr{G}_{(1 \mid 1)}^{(\ell-1) \boldsymbol{e}_{1}}\right)_{\substack{h_{i j m} \rightarrow h_{i j m}-1 / 2 \\
h_{k l m} \rightarrow h_{k l m}-1 / 2}}\right] \\
& +\frac{\ell+d-4}{\ell} \frac{\omega\left(h_{k l m}, \Delta_{m}, \ell\right)}{\omega\left(h_{k l m}, \Delta_{m}, \ell-2\right)} \frac{\left(2 h_{i j m}+\ell\right)\left(2 h_{i j m}+\ell-2+d\right)}{2} \mathscr{G}_{(1 \mid 1)}^{(\ell-2) \boldsymbol{e}_{1} .} .
\end{aligned}
$$

Forgetting about the natural OPE normalization used here and normalizing as is usually done in the literature, we find that the properly-normalized conformal blocks (4.15) and the recurrence relation (4.16) agree with [119], once the $\bar{I}$-functions have been re-expressed in terms of the Exton $G$-function, thus demonstrating that (4.13) is indeed correct.

\subsubsection{Symmetric-traceless exchange in scalar-scalar-scalar- $e_{2}$}

In the previous example, the conformal blocks in the natural OPE basis were computed directly from the pre-conformal blocks for $\ell=0$ and $\ell=1$ and from the general definition for all $\ell$. Here, we will compute the conformal blocks directly in the mixed basis.

For a symmetric-traceless exchange in the four-point correlation function of three scalars and one $\boldsymbol{e}_{2}$, there is only a single tensor structure per OPE; thus, there is only one conformal block per exchanged quasi-primary operator. The tensor structure in the OPE basis is given by

$$
\left({ }_{1} t_{i j}^{12 m}\right)^{E_{1} \cdots E_{\ell} F_{1} \cdots F_{\ell}}=\lambda_{\ell e_{1}}(g)^{\ell} \hat{\mathcal{P}}_{12}^{\ell e_{1}}
$$

where the indices were suppressed on the right-hand side. Meanwhile, the natural threepoint tensor structure is chosen to be

$$
\lambda_{\ell e_{1}} R_{\ell}\left(\overline{\bar{J}}_{34 ; 2}^{\left(d, h_{k l m}, \ell, \Delta_{m}, \ell e_{1}\right)} \cdot{ }_{1} t_{k l m}^{34}\right)_{D_{2} D_{1}\left\{E^{\prime \prime}\right\}}=g_{D_{1} E_{1}^{\prime \prime}} \overline{\bar{\eta}}_{2 D_{2}} \overline{\bar{\eta}}_{4 E_{2}^{\prime \prime}} \cdots \overline{\bar{\eta}}_{4 E_{\ell}^{\prime \prime}},
$$


where $R_{\ell}$ is the appropriate transformation matrix, i.e. the multiplicative factor that normalizes the three-point correlation functions as on the right-hand side.

Using (3.15) and proceeding as in the previous case, the conformal blocks turn out to be

$$
\begin{aligned}
\mathscr{G}_{(1 \mid 1]}^{\ell e_{1}}= & \left({ }_{a} t_{i j}^{12 m}\right)^{E_{1} \cdots E_{\ell} F_{1} \cdots F_{\ell}}\left(x_{3}^{-(\ell-2) / 2} x_{4}^{\ell / 2} \bar{\eta}_{2 D_{2}} \mathcal{A}_{123 E_{1} D_{1}}\left(\mathcal{A}_{123} \cdot \bar{\eta}_{4}\right)_{E_{2}} \cdots\left(\mathcal{A}_{123} \cdot \bar{\eta}_{4}\right)_{E_{\ell}}\right)_{c s_{4}} \\
= & \lambda_{\ell e_{1}} \sum_{i=0}^{\lfloor\ell / 2\rfloor} \frac{(-\ell)_{2 i}}{2^{2 i} i !(-\ell+2-d / 2)_{i}} \mathcal{A}_{12}^{\left(E_{1} E_{2}\right.} \mathcal{A}_{12}^{F_{1} F_{2}} \cdots \mathcal{A}_{12}^{E_{2 i-1} E_{2 i}} \mathcal{A}_{12}^{F_{2 i-1} F_{2 i}} \mathcal{A}_{12}^{E_{2 i+1} F_{2 i+1}} \cdots \mathcal{A}_{12}^{\left.E_{\ell}\right) F_{\ell}} \\
& \times\left(x_{3}^{-(\ell-2) / 2} x_{4}^{\ell / 2} \bar{\eta}_{2 D_{2}} \mathcal{A}_{123 E_{1} D_{1}}\left(\mathcal{A}_{123} \cdot \bar{\eta}_{4}\right)_{E_{2}} \cdots\left(\mathcal{A}_{123} \cdot \bar{\eta}_{4}\right)_{E_{\ell}}\right)_{c s 4} .
\end{aligned}
$$

However, here it is necessary to separate the $E_{1}$ index from the symmetrized set of indices $\{E\}$, since only $\left\{E_{2}, \ldots, E_{\ell}\right\}$ are explicitly symmetrized on the last line of (4.17). Extracting the $E_{1}$ index leads to two different contributions, which would later give two different Gegenbauer polynomials with appropriate conformal substitutions, if it were not for the antisymmetry properties of $\boldsymbol{e}_{2}$. Indeed, one has

$$
\begin{aligned}
\mathscr{G}_{(1 \mid 1]}^{\ell e_{1}}= & \lambda_{\ell e_{1}} \sum_{i=0}^{\lfloor\ell / 2\rfloor} \frac{(-\ell)_{2 i}}{2^{2 i} i !(-\ell+2-d / 2)_{i}} \\
& \times\left[\frac{\ell-2 i}{\ell} \mathcal{A}_{12}^{\left(E_{\ell} E_{2}\right.} \mathcal{A}_{12}^{F_{1} F_{2}} \cdots \mathcal{A}_{12}^{E_{2 i-1} E_{2 i}} \mathcal{A}_{12}^{F_{2 i-1} F_{2 i}} \mathcal{A}_{12}^{E_{2 i+1} F_{2 i+1}} \cdots \mathcal{A}_{12}^{\left.E_{\ell-1}\right) F_{\ell-1}} \mathcal{A}_{12}^{E_{1} F_{\ell}}\right. \\
& \left.+\frac{2 i}{\ell} \mathcal{A}_{12}^{E_{1}\left(E_{2}\right.} \mathcal{A}_{12}^{F_{1} F_{2}} \cdots \mathcal{A}_{12}^{E_{2 i-1} E_{2 i}} \mathcal{A}_{12}^{F_{2 i-1} F_{2 i}} \mathcal{A}_{12}^{E_{2 i+1} F_{2 i+1}} \cdots \mathcal{A}_{12}^{\left.E_{\ell}\right) F_{\ell}}\right] \\
& \times\left(x_{3}^{-(\ell-2) / 2} x_{4}^{\ell / 2} \bar{\eta}_{2 D_{2}} \mathcal{A}_{123 E_{1} D_{1}}\left(\mathcal{A}_{123} \cdot \bar{\eta}_{4}\right)_{E_{2}} \cdots\left(\mathcal{A}_{123} \cdot \bar{\eta}_{4}\right)_{E_{\ell}}\right)_{c s_{4}},
\end{aligned}
$$

where the remaining symmetrization over the set $\left\{E_{2}, \ldots, E_{\ell}\right\}$ can now be neglected. At this point, the computation is completely analogous to the one leading to the conformal blocks for scalar exchange in correlation functions of four scalars, and gives

$$
\begin{aligned}
\mathscr{G}_{(1 \mid 1]}^{\ell e_{1}}= & \lambda_{\ell e_{1}} \sum_{i=0}^{\lfloor\ell / 2\rfloor} \frac{(-2)^{i}(-\ell)_{2 i}}{2^{2 i} i !(-\ell+2-d / 2)_{i}}\left[\frac{\ell-2 i}{\ell} g^{E_{\ell} E_{2}} \bar{\eta}_{1}^{F_{1}} \bar{\eta}_{2}^{F_{2}} \cdots g^{E_{2 i-1} E_{2 i}} \bar{\eta}_{1}^{F_{2 i-1}} \bar{\eta}_{2}^{F_{2 i}} \mathcal{A}_{12}^{E_{2 i+1} F_{2 i+1}} \cdots \mathcal{A}_{12}^{E_{1} F_{\ell}}\right. \\
& \left.+\frac{2 i}{\ell} g^{E_{1} E_{2}} \bar{\eta}_{1}^{F_{1}} \bar{\eta}_{2}^{F_{2}} \cdots g^{E_{2 i-1} E_{2 i}} \bar{\eta}_{1}^{F_{2 i-1}} \bar{\eta}_{2}^{F_{2 i}} \mathcal{A}_{12}^{E_{2 i+1} F_{2 i+1}} \cdots \mathcal{A}_{12}^{E_{\ell} F_{\ell}}\right] \\
& \times\left(x_{3}^{-(\ell-2) / 2} x_{4}^{\ell / 2} \bar{\eta}_{2 D_{2}} \mathcal{A}_{123 E_{1} D_{1}}\left(\mathcal{A}_{123} \cdot \bar{\eta}_{4}\right)_{E_{2}} \cdots\left(\mathcal{A}_{123} \cdot \bar{\eta}_{4}\right)_{E_{\ell}}\right)_{c s_{4}} \\
= & \lambda_{\ell e_{1}} \sum_{i=0}^{\lfloor\ell / 2\rfloor} \frac{(-2)^{i}(-\ell)_{2 i}}{2^{2 i} i !(-\ell+2-d / 2)_{i}}\left[(-2)^{i} \frac{\ell-2 i}{\ell} \bar{\eta}_{1}^{F_{1}} \bar{\eta}_{2}^{F_{2}} \cdots \bar{\eta}_{1}^{F_{2 i-1}} \bar{\eta}_{2}^{F_{2 i}} \mathcal{A}_{12}^{E_{2 i+1} F_{2 i+1}} \cdots \mathcal{A}_{12}^{E_{\ell} F_{\ell}}\right. \\
& \times\left(x_{3}^{-(\ell-2) / 2+i} x_{4}^{\ell / 2-i} \bar{\eta}_{2 D_{2}} \mathcal{A}_{123 E_{\ell} D_{1}}\left(\mathcal{A}_{123} \cdot \bar{\eta}_{4}\right)_{E_{2 i+1}} \cdots\left(\mathcal{A}_{123} \cdot \bar{\eta}_{4}\right)_{E_{\ell-1}}\right)_{c s_{4}} \\
& +(-2)^{i-1} \frac{2 i}{\ell} \bar{\eta}_{1}^{F_{1}} \bar{\eta}_{2}^{F_{2}} \cdots \bar{\eta}_{1}^{F_{2 i-1}} \bar{\eta}_{2}^{F_{2 i}} \mathcal{A}_{12}^{E_{2 i+1} F_{2 i+1}} \cdots \mathcal{A}_{12}^{E_{\ell} F_{\ell}} \\
& \left.\times\left(x_{3}^{-(\ell-2) / 2+i-1} x_{4}^{\ell / 2-i+1} \bar{\eta}_{2 D_{2}}\left(\mathcal{A}_{23} \cdot \bar{\eta}_{4}\right)_{D_{1}}\left(\mathcal{A}_{123} \cdot \bar{\eta}_{4}\right)_{E_{2 i+1}} \cdots\left(\mathcal{A}_{123} \cdot \bar{\eta}_{4}\right)_{E_{\ell}}\right)_{c s_{4}}\right] .
\end{aligned}
$$


Clearly, (4.19) implies two different Gegenbauer polynomials, but the second one has a vanishing coefficient since $\left(\mathcal{A}_{23} \cdot \bar{\eta}_{4}\right)_{D_{1}}$ can be replaced by $-x_{3} \bar{\eta}_{2 D_{1}}$ without loss of generality due to its contraction with the half-projector for $\boldsymbol{e}_{2}$. The antisymmetry of the same halfprojector implies that the second term vanishes, leading to

$$
\begin{aligned}
\mathscr{G}_{(1 \mid 1]}^{\ell e_{1}}= & \lambda_{\ell e_{1}} \sum_{i=0}^{\lfloor\ell / 2\rfloor} \frac{(-1)^{\ell}(\ell-2 i)(-\ell)_{2 i}}{i ! \ell(-\ell+2-d / 2)_{i}} \bar{\eta}_{1}^{F_{1}} \bar{\eta}_{2}^{F_{2}} \cdots \bar{\eta}_{1}^{F_{2 i-1}} \bar{\eta}_{2}^{F_{2 i}} \bar{\eta}_{2}^{E_{2 i+1}} \bar{\eta}_{1}^{F_{2 i+1}} \cdots \bar{\eta}_{2}^{E_{\ell}} \bar{\eta}_{1}^{F_{\ell}} \\
& \times\left(x_{3}^{-(\ell-2) / 2+i} x_{4}^{\ell / 2-i} \bar{\eta}_{2 D_{2}} \mathcal{A}_{123 E_{\ell} D_{1}}\left(\mathcal{A}_{123} \cdot \bar{\eta}_{4}\right)_{E_{2 i+1}} \cdots\left(\mathcal{A}_{123} \cdot \bar{\eta}_{4}\right)_{E_{\ell-1}}\right)_{c s_{4}} \\
= & \lambda_{\ell e_{1}} \frac{(-1)^{\ell}(\ell-1) !}{(d / 2)_{\ell-1}}\left(C_{\ell-1}^{d / 2}(X)\right)_{s},
\end{aligned}
$$

with the conformal substitution

$$
\begin{aligned}
s: & \alpha_{2}^{s_{2}} \alpha_{3}^{s_{3}} \alpha_{4}^{s_{4}} x_{3}^{r_{3}} x_{4}^{r_{4}} \\
\rightarrow & \bar{\eta}_{2 D_{1}} G_{(-1,0,1,1,-1) D_{2}}^{i j|m| k l}-\bar{\eta}_{1 D_{1}} G_{(1,0,3,1,-1) D_{2}}^{i j|m| k l}-x_{3}^{-1} G_{(-1,0,3,3,-1) D_{1} D_{2}}^{i j|m| k l}+G_{(1,0,5,3,-1) D_{1} D_{2}}^{i j|m| k l} \\
= & \rho^{\left(d,\left(\ell-1+s_{2}-s_{3}-s_{4}\right) / 2 ;-h_{i j m}-\ell / 2\right)} x_{3}^{-s_{3}} x_{4}^{-s_{4}} \\
& \times \bar{\eta}_{2 D_{1}} \bar{I}_{12 ; 34}^{\left(d, h_{i j m}-\left(s_{2}-s_{3}-s_{4}+1\right) / 2,1 ;-h_{k l m}+\left(r_{3}-r_{4}+1\right) / 2, \Delta_{m}+h_{k l m}-\left(r_{3}-r_{4}-1\right) / 2\right)}{ }_{D_{2}} \\
& -\rho^{\left(d,\left(\ell+1+s_{2}-s_{3}-s_{4}\right) / 2 ;-h_{i j m}-\ell / 2\right)} x_{3}^{-s_{3}} x_{4}^{-s_{4}} \\
& \times \bar{\eta}_{1 D_{1}} \bar{I}_{12 ; 34}^{\left(d, h_{i j m}-\left(s_{2}-s_{3}-s_{4}+3\right) / 2,1 ;-h_{k l m}+\left(r_{3}-r_{4}+1\right) / 2, \Delta_{m}+h_{k l m}-\left(r_{3}-r_{4}-1\right) / 2\right)}{ }_{D_{2}} \\
= & -\rho^{\left(d,\left(\ell-1+s_{2}-s_{3}-s_{4}\right) / 2 ;-h_{i j m}-\ell / 2\right)} \rho^{\left(d, h_{i j m}-\left(s_{2}-s_{3}-s_{4}+1\right) / 2 ; \Delta_{m}\right)} \\
& \times\left\{1+\frac{\left[\Delta_{m}+h_{i j m}-\left(s_{2}-s_{3}-s_{4}+1\right) / 2\right]\left[-h_{i j m}+\left(s_{2}-s_{3}-s_{4}-1\right) / 2+1-d / 2\right]}{\left[\Delta_{m}+h_{i j m}-\left(s_{2}-s_{3}-s_{4}+3\right) / 2\right]\left[\Delta_{m}+h_{i j m}-\left(s_{2}-s_{3}-s_{4}+3\right) / 2+1-d / 2\right]}\right\} \\
& \times x_{3}^{\Delta_{m}+h_{i j m}-\left(s_{2}+s_{3}-s_{4}+1\right) / 2} x_{4}^{-s_{4}} \bar{\eta}_{1\left[D_{1}\right.} \bar{\eta}_{\left.2 D_{2}\right]} \\
& \times K_{12 ; 34 ; 3}^{\left(d+2, h_{i j m}-\left(s_{2}-s_{3}-s_{4}+1\right) / 2 ;-h_{k l m}+\left(r_{3}-r_{4}+1\right) / 2, \Delta_{m}+h_{k l m}-\left(r_{3}-r_{4}-1\right) / 2\right)}\left(x_{3} ; y_{4}\right),
\end{aligned}
$$

In the first equality of (4.20), a modified version of the argument based on the contiguous relations presented earlier was used to show that $\mathcal{A}_{12}^{E_{\ell} F_{\ell}}$ can nonetheless be replaced by $-\bar{\eta}_{2}^{E_{\ell}} \bar{\eta}_{1}^{F_{\ell}}$. Moreover, in the conformal substitution, all terms symmetric under the interchange of $D_{1}$ and $D_{2}$ were discarded, and the final result was written explicitly in terms of the $K$-function, which is simply the Exton $G$-function. As shown in the first line, without this simplification, the conformal substitution would have four different contributions, originating from the four different terms appearing in $\mathcal{A}_{123 E_{\ell} D_{1}}$.

Finally, it is important to note that the conformal blocks (4.20) exist only for $\ell \geq 1$, as predicted by the tensor product decomposition. Furthermore, as expected from general arguments, the conformal blocks can be expressed with the help of Gegenbauer polynomials written in terms of the variable $X$ (4.14), which is a very convenient feature. Obviously, it is always possible to obtain explicit solutions and recurrence relations for the conformal blocks (4.20), following (4.15) and (4.16) respectively, although it is unnecessary.

\subsubsection{Symmetric-traceless exchange in scalar-vector-scalar-vector}

To elaborate on the mixed basis, we now return to the pre-conformal blocks (4.3) and (4.4) to compute the conformal blocks for symmetric-traceless exchange in scalar-vector-scalarvector four-point correlation functions. 
For scalar exchange, the normalization constant is $\lambda_{\mathbf{0}}=1$ and there is only one tensor structure per OPE, given by $\left({ }_{1} t_{i j}^{12 m}\right)_{B}{ }^{F}=\mathcal{A}_{12 B}{ }^{F} / \sqrt{d}$ and $\left({ }_{1} t_{k l m}^{34}\right)_{D F^{\prime}}=\mathcal{A}_{34 D F^{\prime}} / \sqrt{d}$ respectively. From the pre-conformal block (4.3), we find

$$
\begin{aligned}
\mathscr{G}_{(1 \mid 1)}^{\mathbf{0}} & =\frac{1}{d} \widetilde{K}^{\left(d, h_{k l m}-1 / 2 ; \Delta_{m} ; 0,0,0,1\right)} \mathcal{A}_{12 B}{ }^{F} \mathcal{A}_{34 D F^{\prime}} \bar{I}_{12 ; 34}^{\left(d, h_{i j m}-3 / 2,2 ;-h_{k l m}+1 / 2, \Delta_{m}+h_{k l m}+1 / 2\right)}{ }_{F} F^{\prime} \\
& =\frac{1}{d} \widetilde{K}^{\left(d, h_{k l m}-1 / 2 ; \Delta_{m} ; 0,0,0,1\right)} g_{B}{ }^{F} g_{D F^{\prime}} \bar{I}_{12 ; 34}^{\left(d, h_{i j m}-3 / 2,2 ;-h_{k l m}+1 / 2, \Delta_{m}+h_{k l m}+1 / 2\right)}{ }_{F} F^{\prime} \\
& =\frac{1}{d} \widetilde{K}^{\left(d, h_{k l m}-1 / 2 ; \Delta_{m} ; 0,0,0,1\right)} \bar{I}_{12 ; 34}^{\left(d, h_{i j m}-3 / 2,2 ;-h_{k l m}+1 / 2, \Delta_{m}+h_{k l m}+1 / 2\right)}{ }_{B D} .
\end{aligned}
$$

In the second equality, the transversality of the half-projectors appearing in the four-point correlation function (3.14) was used to simplify the tensor structures.

This result can obviously be expanded in terms of the Exton $G$-function as in (3.16), showing that the conformal block agrees with the one found in the literature [119]. However, since the $\bar{I}$-functions have such nice properties, we do not find it useful to do so.

For vector exchange, there are two tensor structures per OPE, leading to four different conformal blocks. These are given by

$$
\begin{aligned}
& \left({ }_{1} t_{i j}^{12 m}\right)_{B}{ }^{E F_{1} F_{2}}=\sqrt{\frac{2}{(d-1)(d+2)}}\left[\mathcal{A}_{12 B}{ }^{\left(F_{1}\right.} \mathcal{A}_{12}^{\left.F_{2}\right) E}-\frac{1}{d} \mathcal{A}_{12 B}{ }^{E} \mathcal{A}_{12}^{F_{1} F_{2}}\right], \\
& \left({ }_{2} t_{i j}^{12 m}\right)_{B}{ }^{E}=\frac{1}{\sqrt{d}} \mathcal{A}_{12 B}{ }^{E}, \\
& \left({ }_{1} t_{k l m}^{34}\right)_{D E^{\prime} F_{2}^{\prime} F_{1}^{\prime}}=\sqrt{\frac{2}{(d-1)(d+2)}}\left[\mathcal{A}_{34 D\left(F_{1}^{\prime}\right.} \mathcal{A}_{\left.34 F_{2}^{\prime}\right) E^{\prime}}-\frac{1}{d} \mathcal{A}_{34 D E^{\prime}} \mathcal{A}_{34 F_{1}^{\prime} F_{2}^{\prime}}\right] \text {, } \\
& \left({ }_{2} t_{k l m}^{34}\right)_{D E^{\prime}}=\frac{1}{\sqrt{d}} \mathcal{A}_{34 D E^{\prime}}
\end{aligned}
$$

These tensor structures are the natural OPE tensor structures, i.e. they are natural from the point of view of the OPE (3.1). However, they are not the natural three-point function tensor structures, since they do not lead to simple three-point correlation functions. With the normalization constant $\lambda_{\boldsymbol{e}_{1}}=1 / \sqrt{d}$, the latter are computed from

$$
\begin{aligned}
& \lambda_{\boldsymbol{e}_{1}}\left(R_{1}\right)_{1}{ }^{b}\left(\overline{\bar{J}}_{34 ; 2}^{\left(d, h_{k l m}, n_{b}, \Delta_{m}, \boldsymbol{e}_{1}\right)} \cdot{ }_{b} t_{k l m}^{34}\right)_{D E^{\prime \prime}}=\overline{\bar{\eta}}_{2 D} \overline{\bar{\eta}}_{4 E^{\prime \prime}}, \\
& \lambda_{\boldsymbol{e}_{1}}\left(R_{1}\right)_{2}{ }^{b}\left(\overline{\bar{J}}_{34 ; 2}^{\left(d, h_{k l m}, n_{b}, \Delta_{m}, \boldsymbol{e}_{1}\right)} \cdot{ }_{b} t_{k l m}^{34}\right)_{D E^{\prime \prime}}=g_{D E^{\prime \prime}}
\end{aligned}
$$

where the transformation matrix is

$$
\begin{aligned}
R_{1}= & -\frac{\sqrt{d(d-1)(d / 2+1)} \Delta_{m}}{\left(\Delta_{m}-1\right)\left(\Delta_{m}+1-d\right) \rho\left(d, h_{k l m} ; \Delta_{m}\right)} \\
& \times\left(\begin{array}{cc}
\frac{\left(\Delta_{m}-1\right) h_{k l m}+\Delta_{m}\left(\Delta_{m}-d / 2\right)}{2\left(\Delta_{m}+h_{k l m}\right)\left(h_{k l m}\right)_{2}} & \frac{d^{2}+2\left(\Delta_{m}-1\right) h_{k l m}+2 \Delta_{m}^{2}-d\left(2 \Delta_{m}+1\right)}{\sqrt{d(d-1)(d / 2+1)\left(\Delta_{m}+h_{k l m}\right)}} \\
\frac{\Delta_{m}-d / 2}{2\left(h_{k l m}\right)_{2}} & -\frac{(d-2)\left(\Delta_{m}-d\right)}{\sqrt{d(d-1)(d / 2+1)}}
\end{array}\right) .
\end{aligned}
$$

Clearly, the use of both the natural OPE tensor structures ${ }_{a} t_{i j}^{12 m}$ (4.22) and three-point function tensor structures ${ }_{b} t_{k l m}^{34}$ (4.23) in (3.15) simplifies greatly the computation of conformal blocks. Indeed, the simplest conformal blocks are obtained in this mixed basis. 
With the pre-conformal block (4.4), the conformal blocks are thus

$$
\begin{aligned}
\mathscr{G}_{(1 \mid 1]}^{e_{1}}= & \frac{(d-2)\left(h_{i j m}+1\right)\left(2 h_{i j m}+d\right)}{d \sqrt{(d-1)(d / 2+1)}} \\
& \times\left[\bar{I}_{12 ; 34}^{\left(d, h_{i j m}-2,2 ;-h_{k l m}+1, \Delta_{m}+h_{k l m}\right)}{ }_{B D}-\bar{I}_{12 ; 34}^{\left(d, h_{i j m}-2,2 ;-h_{k l m}, \Delta_{m}+h_{k l m}+1\right)}{ }_{B D}\right. \\
& +\frac{2}{d-2}\left(\bar{\eta}_{3 B} \bar{I}_{12 ; 34}^{\left(d, h_{i j m}-1,1 ;-h_{k l m}+1, \Delta_{m}+h_{k l m}\right)}{ }_{D}-\bar{\eta}_{4 B} \bar{I}_{12 ; 34}^{\left(d, h_{i j m}-1,1 ;-h_{k l m}, \Delta_{m}+h_{k l m}+1\right)}{ }_{D}\right) \\
& +\frac{d}{(d-2)\left(h_{i j m}+1\right)\left(2 h_{i j m}+d\right)}\left(\frac{1}{x_{3}} \bar{I}_{12 ; 34}^{\left(d, h_{i j m}-1,2 ;-h_{k l m}+1, \Delta_{m}+h_{k l m}\right)}{ }_{B D}\right. \\
& \left.\left.-\frac{1}{x_{4}} \bar{I}_{12 ; 34}^{\left(d, h_{i j m}-1,2 ;-h_{k l m}, \Delta_{m}+h_{k l m}+1\right)}{ }_{B D}\right)\right], \\
\mathscr{G}_{(1 \mid 2]}^{e_{1}}= & \frac{(d-2)\left(h_{i j m}+1\right)\left(2 h_{i j m}+d\right)}{d \sqrt{(d-1)(d / 2+1)}} \\
& \times\left[\bar{I}_{12 ; 34}^{\left(d, h_{i j m}-2,2 ;-h_{k l m}+1, \Delta_{m}+h_{k l m}\right)}{ }_{B D}-\bar{\eta}_{1 D} \bar{I}_{12 ; 34}^{\left(d, h_{i j m}-1,1 ;-h_{k l m}, \Delta_{m}+h_{k l m}\right)}{ }_{B}\right. \\
& +\frac{2}{d-2}\left(\bar{\eta}_{3 B} \bar{I}_{12 ; 34}^{\left(d, h_{i j m}-1,1 ;-h_{k l m}+1, \Delta_{m}+h_{k l m}\right)}{ }_{D}-g_{B D} \bar{I}_{12 ; 34}^{\left(d, h_{i j m}, 0 ;-h_{k l m}, \Delta_{m}+h_{k l m}\right)}\right) \\
& +\frac{d}{(d-2)\left(h_{i j m}+1\right)\left(2 h_{i j m}+d\right)}\left(\frac{1}{x_{3}} \bar{I}_{12 ; 34}^{\left(d, h_{i j m}-1,2 ;-h_{k l m}+1, \Delta_{m}+h_{k l m}\right)}{ }_{B D}\right. \\
& \left.\left.-\bar{\eta}_{2 D} \bar{I}_{12 ; 34}^{\left(d, h_{i j m}, 1 ;-h_{k l m}, \Delta_{m}+h_{k l m}\right)}{ }_{B}\right)\right],
\end{aligned}
$$

and

$$
\begin{aligned}
\mathscr{G}_{(2 \mid 1]}^{e_{1}}= & \frac{1}{\sqrt{d}}\left[\bar{I}_{12 ; 34}^{\left(d, h_{i j m}-2,2 ;-h_{k l m}+1, \Delta_{m}+h_{k l m}\right)}{ }_{B D}-\bar{I}_{12 ; 34}^{\left(d, h_{i j m}-2,2 ;-h_{k l m}, \Delta_{m}+h_{k l m}+1\right)}{ }_{B D}\right. \\
& \left.-\bar{\eta}_{3 B} \bar{I}_{12 ; 34}^{\left(d, h_{i j m}-1,1 ;-h_{k l m}+1, \Delta_{m}+h_{k l m}\right)}{ }_{D}+\bar{\eta}_{4 B} \bar{I}_{12 ; 34}^{\left(d, h_{i j m}-1,1 ;-h_{k l m}, \Delta_{m}+h_{k l m}+1\right)}{ }_{D}\right], \\
\mathscr{G}_{(2 \mid 2]}^{e_{1}}= & \frac{1}{\sqrt{d}}\left[\bar{I}_{12 ; 34}^{\left(d, h_{i j m}-2,2 ;-h_{k l m}+1, \Delta_{m}+h_{k l m}\right)}{ }_{B D}+g_{B D} \bar{I}_{12 ; 34}^{\left(d, h_{i j m}, 0 ;-h_{k l m}, \Delta_{m}+h_{k l m}\right)}\right. \\
& \left.-\bar{\eta}_{1 D} \bar{I}_{12 ; 34}^{\left(d, h_{i j m}-1,1 ;-h_{k l m}, \Delta_{m}+h_{k l m}\right)}{ }_{B}-\bar{\eta}_{3 B} \bar{I}_{12 ; 34}^{\left(d, h_{i j m}-1,1 ;-h_{k l m}+1, \Delta_{m}+h_{k l m}\right)}{ }_{D}\right],
\end{aligned}
$$

once the transformation matrix $R_{1}$ has been used to rotate to the mixed basis.

The remaining symmetric-traceless exchange can be investigated more straightforwardly from the definition (3.15). In general, there are two tensor structures per OPE, which are simple generalizations of the above, and are given by

$$
\begin{array}{ll}
\left.{ }_{1} t_{i j}^{12 m}\right)_{B}{ }^{E_{1} \cdots E_{\ell} F_{1} \cdots F_{\ell+1}}=\lambda_{(\ell+1) \boldsymbol{e}_{1}}(g)^{\ell} \hat{\mathcal{P}}_{12}^{(\ell+1) \boldsymbol{e}_{1}}, \quad\left({ }_{2} t_{i j}^{12 m}\right)_{B}{ }^{E_{1} \cdots E_{\ell} F_{1} \cdots F_{\ell-1}}=\lambda_{\ell e_{1}}(g)^{\ell} \hat{\mathcal{P}}_{12}^{\ell e_{1}} g, \\
\left.{ }_{(1} t_{k l m}^{34}\right)_{D E_{\ell}^{\prime} \cdots E_{1}^{\prime} F_{\ell+1}^{\prime} \cdots F_{1}^{\prime}}=\lambda_{(\ell+1) \boldsymbol{e}_{1}} \hat{\mathcal{P}}_{34}^{(\ell+1) \boldsymbol{e}_{1}}(g)^{\ell+1}, \quad\left({ }_{2} t_{k l m}^{34}\right)_{D E_{\ell}^{\prime} \cdots E_{1}^{\prime} F_{\ell-1}^{\prime} \cdots F_{1}^{\prime}}=\lambda_{\ell e_{1}} \hat{\mathcal{P}}_{34}^{\ell e_{1}}(g)^{\ell},
\end{array}
$$

where the indices have been suppressed on the right-hand side. Again, these are the natural OPE tensor structures. However, as mentioned above, the conformal blocks are easiest to display in the mixed basis. The relation between the natural three-point function tensor 
structures and the natural OPE tensor structures is given by

$$
\begin{aligned}
& \lambda_{\ell e_{1}}\left(R_{\ell}\right)_{1}{ }^{b}\left(\overline{\bar{J}}_{34 ; 2}^{\left(d, h_{k l m}, n_{b}, \Delta_{m}, \ell e_{1}\right)} \cdot{ }_{b} t_{k l m}^{34}\right)_{D\left\{E^{\prime \prime}\right\}}=\overline{\bar{\eta}}_{2 D} \overline{\bar{\eta}}_{4 E_{1}^{\prime \prime}} \cdots \overline{\bar{\eta}}_{4 E_{\ell}^{\prime \prime}}, \\
& \lambda_{\ell e_{1}}\left(R_{\ell}\right)_{2}{ }^{b}\left(\overline{\bar{J}}_{34 ; 2}^{\left(d, h_{k l m}, n_{b}, \Delta_{m}, \ell e_{1}\right)} \cdot{ }_{b} t_{k l m}^{34}\right)_{D\left\{E^{\prime \prime}\right\}}=g_{D E_{1}^{\prime \prime}} \overline{\bar{\eta}}_{4 E_{2}^{\prime \prime}} \cdots \overline{\bar{\eta}}_{4 E_{\ell}^{\prime \prime}},
\end{aligned}
$$

with the corresponding transformation matrix $R_{\ell}$. Although it is not necessary here, the latter can be easily computed from the three-point correlation functions.

Adapting the steps leading to the conformal blocks for scalar-scalar-scalar-scalar fourpoint correlation functions, while being careful with the explicit symmetrizations appearing in the tensor structures as in the scalar-scalar-scalar- $\boldsymbol{e}_{2}$ four-point correlation functions, the conformal blocks in the mixed basis are given by

$$
\mathscr{G}_{(1 \mid 1]}^{\ell e_{1}}=\lambda_{(\ell+1) e_{1}} \frac{(-1)^{\ell} \ell !}{(d / 2)_{\ell}}\left[\left(C_{\ell}^{d / 2}(X)\right)_{s_{(1 \mid 1)}^{1}}-\left(C_{\ell-1}^{d / 2}(X)\right)_{s_{(1 \mid 1)}^{2}}\right],
$$

with the conformal substitutions

$$
\begin{aligned}
s_{(1 \mid 1)}^{1}: \alpha_{2}^{s_{2}} \alpha_{3}^{s_{3}} \alpha_{4}^{s_{4}} x_{3}^{r_{3}} x_{4}^{r_{4}} & \rightarrow G_{(0,1,3,1,-1) B D}^{i j|m| k l} \\
s_{(1 \mid 1)}^{2}: \alpha_{2}^{s_{2}} \alpha_{3}^{s_{3}} \alpha_{4}^{s_{4}} x_{3}^{r_{3}} x_{4}^{r_{4}} \rightarrow & \bar{\eta}_{4 B} G_{(1,1,2,0,-2) D}^{i j|m| k l}-G_{(1,1,4,0,-2) B D}^{i j|m| k l} \\
& -\bar{\eta}_{3 B} G_{(1,1,2,2,0) D}^{i j|m| k l}+G_{(1,1,4,2,0) B D}^{i j|m| k l}
\end{aligned}
$$

as well as

$$
\begin{aligned}
\mathscr{G}_{(1 \mid 2]}^{\ell e_{1}}= & \lambda_{(\ell+1) e_{1}} \frac{(-1)^{\ell+1}(\ell-1) !}{(d / 2)_{\ell}}\left[\left(C_{\ell-1}^{d / 2}(X)\right)_{s_{(1 \mid 2)}^{1}}-\frac{d}{2}\left(C_{\ell-1}^{d / 2+1}(X)\right)_{s_{(1 \mid 2)}^{2}}\right. \\
& \left.+\frac{d}{2}\left(C_{\ell-2}^{d / 2+1}(X)\right)_{s_{(1 \mid 2)}^{3}}-\frac{d}{2}\left(C_{\ell-3}^{d / 2+1}(X)\right)_{s_{(1 \mid 2)}^{4}}\right]
\end{aligned}
$$

with the conformal substitutions

$$
\begin{aligned}
s_{(1 \mid 2)}^{1}: \alpha_{2}^{s_{2}} \alpha_{3}^{s_{3}} \alpha_{4}^{s_{4}} x_{3}^{r_{3}} x_{4}^{r_{4}} \rightarrow & g_{B D} G_{(1,1,0,0,0)}^{i j|m| k l}-\bar{\eta}_{1 D} G_{(1,1,2,0,0) B}^{i j|m| k l}-\bar{\eta}_{3 B} G_{(1,1,2,2,0) D}^{i j|m| k l}+G_{(1,1,4,2,0) B D}^{i j|m| k l}, \\
s_{(1 \mid 2)}^{2}: \alpha_{2}^{s_{2}} \alpha_{3}^{s_{3}} \alpha_{4}^{s_{4}} x_{3}^{r_{3}} x_{4}^{r_{4}} \rightarrow & \bar{\eta}_{2 D} G_{(-1,1,0,0,0) B}^{i j|m| k l}-\bar{\eta}_{1 D} G_{(1,1,2,0,0) B}^{i j|m| k l}-x_{3}^{-1} G_{(-1,1,2,2,0) B D}^{i j|m| k l}+G_{(1,1,4,2,0) B D}^{i j|m| k l}, \\
s_{(1 \mid 2)}^{3}: \alpha_{2}^{s_{2}} \alpha_{3}^{s_{3}} \alpha_{4}^{s_{4}} x_{3}^{r_{3}} x_{4}^{r_{4}} \rightarrow & \bar{\eta}_{2 D}\left[\bar{\eta}_{4 B} G_{(0,1, k l,-1,-1)}^{i j|m| k}-G_{(0,1,1,-1,-1) B}^{i j|m| k l}-\bar{\eta}_{3 B} G_{(0,1,-1,1,1)}^{i j|m| k l}+G_{(0,1,1,1,1) B}^{i j|m| k l}\right] \\
& -\bar{\eta}_{1 D}\left[\bar{\eta}_{4 B} G_{(2,1,1,-1,-1)}^{i j|m| k l}-G_{(2,1,3,-1,-1) B}^{i j|m| k l}-\bar{\eta}_{3 B} G_{(2,1,1,1,1)}^{i j|m| k l}+G_{(2,1,3,1,1) B}^{i j|m| k l}\right] \\
& -x_{3}^{-1}\left[\bar{\eta}_{4 B} G_{(0,1,1,1,-1) D}^{i j|m| k l}-G_{(0,1,3,1,-1) B D}^{i j|m| k l}-\bar{\eta}_{3 B} G_{(0,1,1,3,1) D}^{i j|m| k l}+G_{(0,1,3,3,1) B D}^{i j|m| k l}\right] \\
& +\bar{\eta}_{4 B} G_{(2,1,3,1,-1) D}^{i j|m| k l}-G_{(2,1,5,1,-1) B D}^{i j|m| k l}-\bar{\eta}_{3 B} G_{(2,1,3,3,1) D}^{i j|m| k l}+G_{(2,1,5,3,1) B D}^{i j|m| k l} \\
& -G_{(0,1,3,1,1,-1) B D}^{i j|m| k l}, \\
s_{(1 \mid 2)}^{4}: \alpha_{2}^{s_{2}} \alpha_{3}^{s_{3}} \alpha_{4}^{s_{4}} x_{3}^{r_{3}} x_{4}^{r_{4}} \rightarrow & \bar{\eta}_{4 B} G_{(1,1,2,0,-2) D}^{i j|m| k l}-G_{(1,1,4,0,-2) B D}^{i j|m| k l}-\bar{\eta}_{3 B} G_{(1,1,2,2,0) D}^{i j|m| k l}+G_{(1,1,4,2,0) B D}^{i j|m| k l},
\end{aligned}
$$

and

$$
\mathscr{G}_{(2 \mid 1]}^{\ell e_{1}}=\lambda_{\ell e_{1}} \frac{(-1)^{\ell-1}(\ell-1) !}{(d / 2)_{\ell-1}}\left[\left(C_{\ell-1}^{d / 2}(X)\right)_{s_{(2 \mid 1)}^{1}}-\left(C_{\ell-2}^{d / 2}(X)\right)_{s_{(2 \mid 1)}^{2}}\right],
$$


with the conformal substitutions

$$
\begin{aligned}
s_{(2 \mid 1)}^{1}: \alpha_{2}^{s_{2}} \alpha_{3}^{s_{3}} \alpha_{4}^{s_{4}} x_{3}^{r_{3}} x_{4}^{r_{4}} \rightarrow & \bar{\eta}_{4 B} G_{(-1,-1,2,0,-2) D}^{i j|m| k l}-G_{(-1,-1,4,0,-2) B D}^{i j|m| k l} \\
& -\bar{\eta}_{3 B} G_{(-1,-1,2,2,0) D}^{i j|m| k l}+G_{(-1,-1,4,2,0) B D}^{i j|m| k l}, \\
s_{(2 \mid 1)}^{2}: \alpha_{2}^{s_{2}} \alpha_{3}^{s_{3}} \alpha_{4}^{s_{4}} x_{3}^{r_{3}} x_{4}^{r_{4}} \rightarrow & G_{(-2,-1,3,1,-1) B D}^{i j|m| k l},
\end{aligned}
$$

and finally

$$
\begin{aligned}
\mathscr{G}_{(2 \mid 2]}^{\ell e_{1}}= & \lambda_{\ell e_{1}} \frac{(-1)^{\ell-1}(\ell-1) !}{\ell(d / 2)_{\ell-1}}\left[\left(C_{\ell-1}^{d / 2}(X)\right)_{s_{(2 \mid 2)}^{1}}+\frac{d}{2}\left(C_{\ell-2}^{d / 2+1}(X)\right)_{s_{(2 \mid 2)}^{2}}\right. \\
& \left.-\frac{d}{2}\left(C_{\ell-3}^{d / 2+1}(X)\right)_{s_{(2 \mid 2)}^{3}}-\left(C_{\ell-2}^{d / 2}(X)-\frac{d}{2} C_{\ell-4}^{d / 2+1}(X)\right)_{s_{(2 \mid 2)}^{4}}\right],
\end{aligned}
$$

with the conformal substitutions

$$
\begin{aligned}
& s_{(2 \mid 2)}^{1}: \alpha_{2}^{s_{2}} \alpha_{3}^{s_{3}} \alpha_{4}^{s_{4}} x_{3}^{r_{3}} x_{4}^{r_{4}} \rightarrow \\
& g_{B D} G_{(-1,-1,0,0,0)}^{i j|m| k l}-\bar{\eta}_{1 D} G_{(-1,-1,2,0,0) B}^{i j|m| k l}-\bar{\eta}_{3 B} G_{(-1,-1,2,2,0) D}^{i j|m| k l}+G_{(-1,-1,4,2,0) B D}^{i j|m| k l}, \\
& s_{(2 \mid 2)}^{2}: \alpha_{2}^{s_{2}} \alpha_{3}^{s_{3}} \alpha_{4}^{s_{4}} x_{3}^{r_{3}} x_{4}^{r_{4}} \rightarrow \\
& \bar{\eta}_{2 D}\left[\bar{\eta}_{4 B} G_{(-2,-1,-1,-1,-1)}^{i j|m| k l}-G_{(-2,-1,1,-1,-1) B}^{i j|m| k l}-\bar{\eta}_{3 B} G_{(-2,-1,-1,1,1)}^{i j|m| k l}+G_{(-2,-1,1,1,1) B}^{i j|m| k l}\right] \\
& -\bar{\eta}_{1 D}\left[\bar{\eta}_{4 B} G_{(0,-1,1,-1,-1)}^{i j|m| k l}-G_{(0,-1,3,-1,-1) B}^{i j|m| k l}-\bar{\eta}_{3 B} G_{(0,-1,1,1,1)}^{i j|m| k l}+G_{(0,-1,3,1,1) B}^{i j|m| k l}\right] \\
& -x_{3}^{-1}\left[\bar{\eta}_{4 B} G_{(-2,-1,1,1,-1) D}^{i j|m| k l}-G_{(-2,-1,3,1,-1) B D}^{i j|m| k l}-\bar{\eta}_{3 B} G_{(-2,-1,1,3,1) D}^{i j|m| k l}+G_{(-2,-1,3,3,1) B D}^{i j|m| k l}\right] \\
& +\bar{\eta}_{4 B} G_{(0,-1,3,1,-1) D}^{i j|m| k l}-G_{(0,-1,5,1,-1) B D}^{i j|m| k l}-\bar{\eta}_{3 B} G_{(0,-1,3,3,1) D}^{i j|m| k l}+G_{(0,-1,5,3,1) B D}^{i j|m| k l}, \\
& s_{(2 \mid 2)}^{3}: \alpha_{2}^{s_{2}} \alpha_{3}^{s_{3}} \alpha_{4}^{s_{4}} x_{3}^{r_{3}} x_{4}^{r_{4}} \rightarrow \\
& \bar{\eta}_{2 D} G_{(-3,-1,0,0,0) B}^{i j|m| k l}-\bar{\eta}_{1 D} G_{(-1,-1,2,0,0) B}^{i j|m| k l}-x_{3}^{-1} G_{(-3,-1,2,2,0) B D}^{i j|m| k l}+G_{(-1,-1,4,2,0) B D}^{i j|m| k l} \\
& +\left[\bar{\eta}_{4 B} G_{(-1,-1,2,0,-2) D}^{i j|m| k l}-G_{(-1,-1,4,0,-2) B D}^{i j|m| k l}-\bar{\eta}_{3 B} G_{(-1,-1,2,2,0) D}^{i j|m| k l}+G_{(-1,-1,4,2,0) B D}^{i j|m| k l}\right], \\
& s_{(2 \mid 2)}^{4}: \alpha_{2}^{s_{2}} \alpha_{3}^{s_{3}} \alpha_{4}^{s_{4}} x_{3}^{r_{3}} x_{4}^{r_{4}} \rightarrow G_{(-2,-1,3,1,-1) B D}^{i j|m| k l} .
\end{aligned}
$$

Clearly, only (4.26) exists for $\ell=0$ and matches with (4.21) once the proper rescaling necessary to convert from the purely OPE basis of the latter to the mixed basis of the former is done. Moreover, for $\ell=1$, all the conformal blocks (4.26), (4.27), (4.28) and (4.29) match the conformal blocks (4.24) and (4.25) obtained from the pre-conformal blocks rotated to the mixed basis. Finally, as for all previous four-point correlation functions, the conformal blocks are easily displayed as Gegenbauer polynomials in terms of the variable $X$ (4.14). They can be expanded explicitly as in (4.15), and recurrence relations can be found as in (4.16).

Before proceeding, it is interesting to note the similarities between the conformal blocks (4.26) and (4.28) and their respective conformal substitutions. As can be seen above, other similarities occur, mostly due to their common origin, mainly the OPE. Moreover, in $s_{(2 \mid 2)}^{3}$ the two terms in red merge. They were kept separate to exhibit the similarities in the overall rule. 


\subsection{4 $\ell e_{1}+e_{2}$ exchange in scalar-vector-scalar-vector}

In the case of $\ell \boldsymbol{e}_{1}+\boldsymbol{e}_{2}$ exchange in scalar-vector-scalar-vector four-point correlation functions, there is only one tensor structure per OPE. As before, the easiest way to obtain the conformal blocks is to work in the mixed basis. The OPE and three-point tensor structures are simply

$$
\begin{aligned}
& \left({ }_{1} t_{i j}^{12 m}\right)_{B}{ }^{E_{1} \cdots E_{\ell+2} F_{1} \cdots F_{\ell+1}}=\lambda_{\ell e_{1}+e_{2}}\left((g)^{\ell+2} \hat{\mathcal{P}}_{12}^{\ell e_{1}+e_{2}} g\right)^{E_{1} \cdots E_{\ell+2} F_{\ell+1} \cdots F_{1}}{ }_{B}, \\
& \lambda_{\ell e_{1}+e_{2}} R_{\ell}\left(\bar{J}_{34 ; 2}^{\left(d, h_{k l m}, \ell+1, \Delta_{m}, \ell e_{1}+e_{2}\right)} \cdot{ }_{1} t_{k l m}^{34}\right)_{D\left\{E^{\prime \prime}\right\}}=g_{D E_{1}^{\prime \prime}} \overline{\bar{\eta}}_{4 E_{2}^{\prime \prime}} \cdots \overline{\bar{\eta}}_{4 E_{\ell+2}^{\prime \prime}},
\end{aligned}
$$

where on the right-hand side the indices $B$ and $F_{1}$ are matched to $E_{1}$ and $E_{2}$, respectively, and $R_{\ell}$ is the transformation matrix which is just a multiplicative factor introduced for proper normalization of the three-point correlation functions. It is understood that $E_{1}$ and $E_{2}$ (respectively $B$ and $F_{1}$ ) are the $\boldsymbol{e}_{2}$ indices of the $\ell \boldsymbol{e}_{1}+\boldsymbol{e}_{2}$, hence they are antisymmetrized as in (4.5).

Following the arguments presented above, it is easy to obtain

$$
\begin{aligned}
\mathscr{G}_{(1 \mid 1]}^{\ell e_{1}+e_{2}}= & \lambda_{\ell e_{1}+e_{2}} \frac{(-1)^{\ell+1} 2 \ell !}{(\ell+2)(d / 2)_{\ell}} \\
& \times\left[\left(X C_{\ell}^{d / 2}(X)-\frac{2 \ell-2+3 d / 2}{\ell-2+d} C_{\ell-1}^{d / 2}(X)+\frac{d}{2} X^{2} C_{\ell-1}^{d / 2+1}(X)-d X C_{\ell-2}^{d / 2+1}(X)+\frac{d}{2} C_{\ell-3}^{d / 2+1}(X)\right)_{s_{1}}\right. \\
& -\frac{1}{2}\left(C_{\ell}^{d / 2}(X)+\frac{d}{2} X C_{\ell-1}^{d / 2+1}(X)-\frac{d(d / 2-2)}{\ell-2+d} C_{\ell-2}^{d / 2+1}(X)\right)_{s_{2}} \\
& +\frac{1}{2}\left(\frac{2 \ell-2+3 d / 2}{\ell-2+d} C_{\ell-1}^{d / 2}(X)+\frac{d(\ell+d / 2)}{\ell-2+d} X C_{\ell-2}^{d / 2+1}(X)-\frac{d}{2} C_{\ell-3}^{d / 2+1}(X)\right)_{s_{3}} \\
& \left.-\frac{1}{2}\left(\frac{2 \ell-2+3 d / 2}{\ell-2+d} X C_{\ell-1}^{d / 2}(X)+\frac{d(\ell+d / 2)}{\ell-2+d} X^{2} C_{\ell-2}^{d / 2+1}(X)-\frac{d}{2} X C_{\ell-3}^{d / 2+1}(X)\right)\right],
\end{aligned}
$$

with the conformal substitutions

$$
\begin{aligned}
& s_{1}: \alpha_{2}^{s_{2}} \alpha_{3}^{s_{3}} \alpha_{4}^{s_{4}} x_{3}^{r_{3}} x_{4}^{r_{4}} \rightarrow g_{B D} G_{(1,1,0,0,0)}^{i j|m| k l}-\bar{\eta}_{1 D} G_{(1,1,2,0,0) B}^{i j|m| k l}-\bar{\eta}_{3 B} G_{(1,1,2,2,0) D}^{i j|m| k l}+G_{(1,1,4,2,0) B D}^{i j|m| k l} \\
& s_{2}: \alpha_{2}^{s_{2}} \alpha_{3}^{s_{3}} \alpha_{4}^{s_{4}} x_{3}^{r_{3}} x_{4}^{r_{4}} \rightarrow \bar{\eta}_{2 D}\left[\bar{\eta}_{4 B} G_{(0,1,-1,-1,-1)}^{i j|m| k l}-G_{(0,1,1,-1,-1) B}^{i j|m| k l}-\bar{\eta}_{3 B} G_{(0,1,-1,1,1)}^{i j|m| k l}+G_{(0,1,1,1,1) B}^{i j|m| k l}\right] \\
& -\bar{\eta}_{1 D}\left[\bar{\eta}_{4 B} G_{(2,1,1,-1,-1)}^{i j|m| k l}-G_{(2,1,3,-1,-1) B}^{i j|m| k l}-\bar{\eta}_{3 B} G_{(2,1,1,1,1)}^{i j|m| k l}+G_{(2,1,3,1,1) B}^{i j|m| k l}\right] \\
& -x_{3}^{-1}\left[\bar{\eta}_{4 B} G_{(0,1,1,1,-1) D}^{i j|m| k l}-G_{(0,1,3,1,-1) B D}^{i j|m| k l}-\bar{\eta}_{3 B} G_{(0,1,1,3,1) D}^{i j|m| k l}+G_{(0,1,3,3,1) B D}^{i j|m| k l}\right] \\
& +\bar{\eta}_{4 B} G_{(2,1,3,1,-1) D}^{i j|m| k l}-G_{(2,1,5,1,-1) B D}^{i j|m| k l}-\bar{\eta}_{3 B} G_{(2,1,3,3,1) D}^{i j|m| k l}+G_{(2,1,5,3,1) B D}^{i j|m| k l}, \\
& s_{3}: \alpha_{2}^{s_{2}} \alpha_{3}^{s_{3}} \alpha_{4}^{s_{4}} x_{3}^{r_{3}} x_{4}^{r_{4}} \rightarrow \bar{\eta}_{4 B} G_{(1,1,2,0,-2) D}^{i j|m| k l}-G_{(1,1,4,0,-2) B D}^{i j|m| k l}-\bar{\eta}_{3 B} G_{(1,1,2,2,0) D}^{i j|m| k l}+G_{(1,1,4,2,0) B D}^{i j|m| k l} \\
& +\bar{\eta}_{2 D} G_{(-1,1,0,0,0) B}^{i j|m| k l}-\bar{\eta}_{1 D} G_{(1,1,2,0,0) B}^{i j|m| k l}-x_{3}^{-1} G_{(-1,1,2,2,0) B D}^{i j|m| k l}+G_{(1,1,4,2,0) B D}^{i j|m| k l}, \\
& s_{4}: \alpha_{2}^{s_{2}} \alpha_{3}^{s_{3}} \alpha_{4}^{s_{4}} x_{3}^{r_{3}} x_{4}^{r_{4}} \rightarrow G_{(0,1,3,1,-1) B D}^{i j|m| k l}
\end{aligned}
$$

The observation that the coefficients in the projection operator for $\ell \boldsymbol{e}_{1}+\boldsymbol{e}_{2}$ are related to those in the projection operator for $\ell \boldsymbol{e}_{1}$ and the fact that the latter lead to Gegenbauer polynomials explain why all conformal blocks can be displayed as appropriate conformal substitutions of Gegenbauer polynomials in the variable $X$. 


\subsubsection{Symmetric-traceless exchange in scalar-scalar-vector-vector}

For completeness, in our final example, we determine the conformal blocks for scalarscalar-vector-vector four-point correlation functions, which would then empower us to fully implement the bootstrap for correlation functions of two scalars and two vectors.

In the mixed basis, the necessary inputs are the tensor structures for symmetrictraceless exchange, which are

$$
\begin{aligned}
\left({ }_{1} t_{i j}^{12 m}\right)^{E_{1} \cdots E_{\ell} F_{1} \cdots F_{\ell}} & =\lambda_{\ell e_{1}}(g)^{\ell} \hat{\mathcal{P}}_{12}^{\ell e_{1}}, \\
\lambda_{\ell e_{1}}\left(R_{\ell}\right)_{1}{ }^{b}\left(\overline{\bar{J}}_{34 ; 2}^{\left(d, h_{k l m}, n_{b}, \Delta_{m}, \ell e_{1}\right)} \cdot{ }_{b} t_{k l m}^{34}\right)_{C D\left\{E^{\prime \prime}\right\}} & =\overline{\bar{\eta}}_{2 C} \overline{\bar{\eta}}_{2 D} \overline{\bar{\eta}}_{4 E_{1}^{\prime \prime}} \cdots \overline{\bar{\eta}}_{4 E_{\ell}^{\prime \prime}}, \\
\lambda_{\ell e_{1}}\left(R_{\ell}\right)_{2}{ }^{b}\left(\overline{\bar{J}}_{34 ; 2}^{\left(d, h_{k l m}, n_{b}, \Delta_{m}, \ell e_{1}\right)} \cdot{ }_{b} t_{k l m}^{34}\right)_{C D\left\{E^{\prime \prime}\right\}} & =g_{C D} \overline{\bar{\eta}}_{4 E_{1}^{\prime \prime}} \cdots \overline{\bar{\eta}}_{4 E_{\ell}^{\prime \prime}}, \\
\lambda_{\ell e_{1}}\left(R_{\ell}\right)_{3}{ }^{b}\left(\overline{\bar{J}}_{34 ; 2}^{\left(d, h_{k l m}, n_{b}, \Delta_{m}, \ell e_{1}\right)} \cdot{ }_{b} t_{k l m}^{34}\right)_{C D\left\{E^{\prime \prime}\right\}} & =g_{C E_{1}^{\prime \prime}} \overline{\bar{\eta}}_{2 D} \overline{\bar{\eta}}_{4 E_{2}^{\prime \prime}} \cdots \overline{\bar{\eta}}_{4 E_{\ell}^{\prime \prime}}, \\
\lambda_{\ell e_{1}}\left(R_{\ell}\right)_{4}{ }^{b}\left(\overline{\bar{J}}_{34 ; 2}^{\left(d, h_{k l m}, n_{b}, \Delta_{m}, \ell e_{1}\right)} \cdot{ }_{b} t_{k l m}^{34}\right)_{C D\left\{E^{\prime \prime}\right\}} & =g_{D E_{1}^{\prime \prime}} \overline{\bar{\eta}}_{2 C} \overline{\bar{\eta}}_{4 E_{2}^{\prime \prime}} \cdots \overline{\bar{\eta}}_{4 E_{\ell}^{\prime \prime}}, \\
\lambda_{\ell e_{1}}\left(R_{\ell}\right)_{5}{ }^{b}\left(\overline{\bar{J}}_{34 ; 2}^{\left(d, h_{k l m}, n_{b}, \Delta_{m}, \ell e_{1}\right)} \cdot{ }_{b} t_{k l m}^{34}\right)_{C D\left\{E^{\prime \prime}\right\}} & =g_{C E_{1}^{\prime \prime}} g_{D E_{2}^{\prime \prime}} \overline{\bar{\eta}}_{4 E_{3}^{\prime \prime}} \cdots \overline{\bar{\eta}}_{4 E_{\ell}^{\prime \prime}} \cdot
\end{aligned}
$$

Once again, the indices were suppressed on the right-hand side of the natural OPE tensor structure, and the transformation matrix $R_{\ell}$ leads to the natural three-point tensor structures.

The conformal blocks are thus

$$
\mathscr{G}_{(1 \mid 1]}^{\ell e_{1}}=\lambda_{\ell e_{1}} \frac{(-1)^{\ell} \ell !}{(d / 2-1)_{\ell}}\left(C_{\ell}^{d / 2-1}(X)\right)_{s_{(1 \mid 1)}},
$$

with the conformal substitution

$$
s_{(1 \mid 1)}: \alpha_{2}^{s_{2}} \alpha_{3}^{s_{3}} \alpha_{4}^{s_{4}} x_{3}^{r_{3}} x_{4}^{r_{4}} \rightarrow G_{(0,0,4,2,-2) C D}^{i j|m| k l},
$$

followed by

$$
\mathscr{G}_{(1 \mid 2]}^{\ell e_{1}}=\lambda_{\ell e_{1}} \frac{(-1)^{\ell} \ell !}{(d / 2-1)_{\ell}}\left(C_{\ell}^{d / 2-1}(X)\right)_{s_{(1 \mid 2)}},
$$

with the conformal substitution

$$
s_{(1 \mid 2)}: \alpha_{2}^{s_{2}} \alpha_{3}^{s_{3}} \alpha_{4}^{s_{4}} x_{3}^{r_{3}} x_{4}^{r_{4}} \rightarrow g_{C D} G_{(0,0,0,0,0)}^{i j|m| k l},
$$

as well as

$$
\mathscr{G}_{(1 \mid 3]}^{\ell e_{1}}=\lambda_{\ell e_{1}} \frac{(-1)^{\ell}(\ell-1) !}{(d / 2)_{\ell-1}}\left[\left(C_{\ell-1}^{d / 2}(X)\right)_{s_{(1 \mid 3)}^{1}}-\left(C_{\ell-2}^{d / 2}(X)\right)_{s_{(1 \mid 3)}^{2}}\right],
$$

with the conformal substitutions

$$
\begin{aligned}
s_{(1 \mid 3)}^{1}: \alpha_{2}^{s_{2}} \alpha_{3}^{s_{3}} \alpha_{4}^{s_{4}} x_{3}^{r_{3}} x_{4}^{r_{4}} \rightarrow & \bar{\eta}_{2 C} G_{(-1,0,1,1,-1) D}^{i j|m| k l}-\bar{\eta}_{1 C} G_{(1,0,3,1,-1) D}^{i j|m| k l} \\
& -x_{3}^{-1} G_{(-1,0,3,3,-1) C D}^{i j|m| k l}+G_{(1,0, k, 3,-1) C}^{i j|m| k l} \\
s_{(1 \mid 3)}^{2}: \alpha_{2}^{s_{2}} \alpha_{3}^{s_{3}} \alpha_{4}^{s_{4}} x_{3}^{r_{3}} x_{4}^{r_{4}} \rightarrow & G_{(0,0,4,2,-2) C D}^{i j|m| k l},
\end{aligned}
$$


and

$$
\mathscr{G}_{(1 \mid 4]}^{\ell e_{1}}=\lambda_{\ell e_{1}} \frac{(-1)^{\ell}(\ell-1) !}{(d / 2)_{\ell-1}}\left[\left(C_{\ell-1}^{d / 2}(X)\right)_{s_{(1 \mid 4)}^{1}}-\left(C_{\ell-2}^{d / 2}(X)\right)_{s_{(1 \mid 4)}^{2}}\right],
$$

with the conformal substitutions

$$
\begin{aligned}
s_{(1 \mid 4)}^{1}: \alpha_{2}^{s_{2}} \alpha_{3}^{s_{3}} \alpha_{4}^{s_{4}} x_{3}^{r_{3}} x_{4}^{r_{4}} \rightarrow & \bar{\eta}_{2 D} G_{(-1,0,1,1,-1) C}^{i j|m| k l}-\bar{\eta}_{1 D} G_{(1,0,3,1,-1) C}^{i j|m| k l} \\
& -x_{3}^{-1} G_{(-1,0,3,3,-1) C D}^{i j|m| k l}+G_{(1,0,5,3,-1) C D}^{i j|m| k l} \\
s_{(1 \mid 4)}^{2}: \alpha_{2}^{s_{2}} \alpha_{3}^{s_{3}} \alpha_{4}^{s_{4}} x_{3}^{r_{3}} x_{4}^{r_{4}} \rightarrow & G_{(0,0,4,2,-2) C D}^{i j|m| k l}
\end{aligned}
$$

and finally

$$
\begin{aligned}
\mathscr{G}_{(1 \mid 5]}^{\ell e_{1}}= & \lambda_{\ell e_{1}} \frac{(-1)^{\ell}(\ell-2) !}{(d / 2)_{\ell-1}}\left[\left(C_{\ell-2}^{d / 2}(X)\right)_{s_{(1 \mid 5)}^{1}}+\frac{d}{2}\left(C_{\ell-2}^{d / 2+1}(X)\right)_{s_{(1 \mid 5)}^{2}}\right. \\
& \left.-\frac{d}{2}\left(C_{\ell-3}^{d / 2+1}(X)\right)_{s_{(1 \mid 5)}^{3}}+\frac{d}{2}\left(C_{\ell-4}^{d / 2+1}(X)\right)_{s_{(1 \mid 5)}^{4}}\right]
\end{aligned}
$$

with the conformal substitutions

$$
\begin{aligned}
s_{(1 \mid 5)}^{1}: \alpha_{2}^{s_{2}} \alpha_{3}^{s_{3}} \alpha_{4}^{s_{4}} x_{3}^{r_{3}} x_{4}^{r_{4}} \rightarrow & g_{C D} G_{(0,0,0,0,0)}^{i j|m| k l}, \\
s_{(1 \mid 5)}^{2}: \alpha_{2}^{s_{2}} \alpha_{3}^{s_{3}} \alpha_{4}^{s_{4}} x_{3}^{r_{3}} x_{4}^{r_{4}} \rightarrow & \bar{\eta}_{2 C}\left[\bar{\eta}_{2 D} G_{(-2,0,-2,0,0)}^{i j|m| k l}-\bar{\eta}_{1 D} G_{(0,0,0,0,0)}^{i j|m| k l}-x_{3}^{-1} G_{(-2,0,0,2,0) D}^{i j|m| k}+G_{(0,0,2,2,0) D}^{i j|m| k l}\right] \\
& -\bar{\eta}_{1 C}\left[\bar{\eta}_{2 D} G_{(0,0,0,0,0)}^{i j|m| k l}-\bar{\eta}_{1 D} G_{(2,0,2,0,0)}^{i j|m| k l}-x_{3}^{-1} G_{(0,0,2,2,0) D}^{i j|m| k l}+G_{(2,0,4,2,0) D}^{i j|m| k}\right] \\
& -x_{3}^{-1}\left[\bar{\eta}_{2 D} G_{(-2,0,0,2,0) C}^{i j|m| k l}-\bar{\eta}_{1 D} G_{(0,0,2,2,0) C}^{i j|m| k l}-x_{3}^{-1} G_{(-2,0,2,4,0) C D}^{i j|m| k}+G_{(0,0,4,4,0) C D}^{i j|m| k l}\right] \\
& +\bar{\eta}_{2 D} G_{(0,0,2,2,0) C}^{i j|m| k l}-\bar{\eta}_{1 D} G_{(2,0,4,2,0) C}^{i j|m| k l}-x_{3}^{-1} G_{(0,0), 4,4,0) C D}^{i j|m| k l}+G_{(2,0,6,4,0) C D}^{i j|m| k l}, \\
s_{(1 \mid 5)}^{3}: \alpha_{2}^{s_{2}} \alpha_{3}^{s_{3}} \alpha_{4}^{s_{4}} x_{3}^{r_{3}} x_{4}^{r_{4}} \rightarrow & \bar{\eta}_{2 D} G_{(-1,0,1,1,-1) C}^{i j|m| k l}-\bar{\eta}_{1 D} G_{(1,0,3,1,-1) C}^{i j|m| k l}-x_{3}^{-1} G_{(-1,0,3,3,-1) C D}^{i j|m| k l}+G_{(1,0,5,3,-1) C D}^{i j \mid m l} \\
& +\{C \leftrightarrow D\}, \\
s_{(1 \mid 5)}^{4}: \alpha_{2}^{s_{2}} \alpha_{3}^{s_{3}} \alpha_{4}^{s_{4}} x_{3}^{r_{3}} x_{4}^{r_{4}} \rightarrow & G_{(0,0,4,2,-2) C D}^{i j|m| k l} .
\end{aligned}
$$

In this example, there are two conformal blocks for $\ell=0$, four conformal blocks for $\ell=1$, and five conformal blocks for $\ell>1$, as expected from the tensor product decomposition.

\subsubsection{Conformal blocks as linear combinations of Gegenbauer polynomials with substitutions}

All of the examples above led to expressions for conformal blocks given by linear combinations of Gegenbauer polynomials with appropriate conformal substitutions. On the one hand, noting the identical simplifications that occur in the procedure leading to the conformal blocks, we anticipate that there are generic Feynman-like rules for the corresponding conformal substitutions that can be deduced from the previous examples, starting from the mixed basis.

On the other hand, the presence of Gegenbauer polynomials in terms of the variable $X$ might at first seem intriguing. The origin of the variable $X$ is clear, as it is directly obtained from the $\mathcal{A}$-metric contractions. We can also argue that the Gegenbauer polynomials appear for any tower of conformal blocks with exchanged quasi-primary operators 
in $\boldsymbol{N}+\ell \boldsymbol{e}_{1}$. Indeed, starting from the mixed basis, the three-point correlation function does not have any special features. Then, the three-point function is multiplied by hatted projection operators at different embedding space coordinates. In [174], it was proved that the hatted projection operators merged into one hatted projection operator constructed from the two $\mathcal{A}$-metrics. Subsequently, the result is transformed into the conformal block with the help of the conformal substitution (3.13) and contractions with the tensor structure. At this point, the implicit hatted projection operator can be extracted from the tensor structure, as described in [174], moving all the nontrivial $\ell$-dependence to the hatted projection operators. Now, from the tensor product decomposition, we know that the hatted projection operator for $\boldsymbol{N}+\ell \boldsymbol{e}_{1}$ can be obtained from the tensor product of $\boldsymbol{N}$ and $\ell \boldsymbol{e}_{1}$. In that product, one must subtract the smaller irreducible representations. The trace ones are easily discarded, while the non-trace ones can be removed by simply demanding that the resulting projection operator satisfy the proper symmetries. Hence, the hatted projection operator for $\boldsymbol{N}+\ell \boldsymbol{e}_{1}$ is built from the fixed projection operator for $\boldsymbol{N}$ and the projection operator for $\ell \boldsymbol{e}_{1}$. The latter carries the $\ell$-dependence through its coefficients, see (4.1). The coefficients, which re-sum into simple Gegenbauer polynomials, ultimately lead to linear combinations of Gegenbauer polynomials after the steps necessary to determine the conformal substitutions are completed. Hence, in a fixed four-point correlation function, conformal blocks for a tower of quasi-primary operators in irreducible representations $\boldsymbol{N}+\ell \boldsymbol{e}_{1}$ are expressed as linear combinations of Gegenbauer polynomials with proper conformal substitutions, in agreement with the examples above. Moreover, the conformal substitutions replace the variable $X$ by $\bar{I}$-functions, which are tensorial generalizations of the Exton $G$-function, without derivatives.

\section{Discussion and conclusion}

We have shown how to obtain conformal blocks using the method described in $[1,2]$. Given the agreement with several results in the literature obtained using other methods, and our earlier calculations of two- and three-point functions in [173, 174], it is clear that the approach is sound. Using the OPE in embedding space, one can indeed systematically build up $M$-point functions from $(M-1)$-point functions and so on and obtain explicit expressions for $M$-point functions. As we have already stressed, the method is universal and is not limited to any particular Lorentz representation or spacetime dimension.

This claim is that the result (3.15) for the conformal blocks is completely general, with the conformal substitution rules given by (3.5) and (3.13), respectively. All that one needs to supply in order to determine a particular conformal block of interest are some grouptheoretic quantities, namely the projection operators for the exchanged representations and the tensor structures. The method rests on the embedding space OPE framework, which was carefully developed in $[1,2]$ and was subsequently placed on a firmer footing in $[173,174]$. The examples described above serve to illustrate the application of the method in practice. In each case, one first determines the form of the three-point tensor structures for the left and right OPE and then supplies the appropriate projector for the exchanged operator. Together, these objects comprise all the input data needed for a specific block. 
Next, one directly inserts this data into the general formula for the conformal blocks given in (3.15) and extracts the relevant linear combination of Gegenbauer polynomials, with each term coupled to its associated substitution rule. That is the overall idea behind the universal formula presented here. The method is general, because arbitrary Lorentz representations may be considered, and the only components which vary from case to case are the group-theoretic input data.

Now, the general procedure for obtaining conformal blocks is described in section 3 , and it involves starting with the hatted projection operators and then performing substitutions. The two required substitutions involve first the three-point tensorial function and then the four-point tensorial function on the outcome of the first substitution. Carrying out the conformal substitutions is straightforward but can become tedious for four-point correlation functions of quasi-primary operators in large irreducible representations. Obtaining the hatted projection operators is perhaps less straightforward, but can also be done systematically by starting with small representations and then working up to larger ones. It is likely that the procedure for computing conformal blocks could be automated and handled by a computer program.

The intermediate expressions for the blocks involving the Gegenbauer polynomials, which lead to the actual conformal blocks through the $s$-substitutions, are certainly intriguing. Based on the examples we have worked out, we argued that this feature is general and applicable to other conformal blocks. Moreover, it should be possible to codify the procedure for obtaining the appropriate conformal substitutions as a set of Feynman-like rules. This will be addressed in a future publication.

While many of the conformal blocks derived here were already known, it was useful to rederive such results using the new method. Looking ahead to what can be accomplished with this method soon, we consider tackling several specific conformal blocks. One of the most fundamental objects for exploring CFTs is the four-point function involving four energy-momentum tensors. Because of the sheer number of blocks involved in a four-point function of energy-momentum tensors, this will be the subject of a separate publication.

Looking further ahead, we hope that our approach will be useful for both the numerical and analytic bootstrap. Despite an enormous amount of progress, it is apparent that CFTs have a rich and complicated structure that has not been fully explored yet.

\section{Acknowledgments}

The authors would like to thank Wen-Jie Ma for useful discussions. The work of JFF and VP is supported by NSERC and FRQNT. JFF would like to thank the organizers and participants of the Bootstrap 2019 conference, held at Perimeter Institute, for their hospitality. WS would like to thank the KITP Santa Barbara for its hospitality, his work was supported in part by the National Science Foundation under Grant No. NSF PHY1748958. VP would liked to thank the Aspen Center for Physics (ACP), where part of this work was performed. The ACP is supported by National Science Foundation grant PHY-1607611. 
Open Access. This article is distributed under the terms of the Creative Commons Attribution License (CC-BY 4.0), which permits any use, distribution and reproduction in any medium, provided the original author(s) and source are credited.

\section{References}

[1] J.-F. Fortin and W. Skiba, A recipe for conformal blocks, arXiv:1905.00036 [INSPIRE].

[2] J.-F. Fortin and W. Skiba, New methods for conformal correlation functions, JHEP 06 (2020) 028 [arXiv: 1905.00434] [inSPIRE].

[3] S. Ferrara, A.F. Grillo and R. Gatto, Tensor representations of conformal algebra and conformally covariant operator product expansion, Annals Phys. 76 (1973) 161 [INSPIRE].

[4] A.M. Polyakov, Nonhamiltonian approach to conformal quantum field theory, Zh. Eksp. Teor. Fiz. 66 (1974) 23 [INSPIRE].

[5] R. Rattazzi, V.S. Rychkov, E. Tonni and A. Vichi, Bounding scalar operator dimensions in 4D CFT, JHEP 12 (2008) 031 [arXiv:0807.0004] [INSPIRE].

[6] V.S. Rychkov and A. Vichi, Universal Constraints on Conformal Operator Dimensions, Phys. Rev. D 80 (2009) 045006 [arXiv:0905.2211] [INSPIRE].

[7] F. Caracciolo and V.S. Rychkov, Rigorous Limits on the Interaction Strength in Quantum Field Theory, Phys. Rev. D 81 (2010) 085037 [arXiv:0912.2726] [InSPIRE].

[8] D. Poland and D. Simmons-Duffin, Bounds on 4D Conformal and Superconformal Field Theories, JHEP 05 (2011) 017 [arXiv: 1009.2087] [InSPIRE].

[9] R. Rattazzi, S. Rychkov and A. Vichi, Central Charge Bounds in $4 D$ Conformal Field Theory, Phys. Rev. D 83 (2011) 046011 [arXiv: 1009.2725] [InSPIRE].

[10] D. Poland, D. Simmons-Duffin and A. Vichi, Carving Out the Space of 4 D CFTs, JHEP 05 (2012) 110 [arXiv:1109.5176] [INSPIRE].

[11] S. Rychkov, Conformal Bootstrap in Three Dimensions?, arXiv:1111.2115 [INSPIRE].

[12] S. El-Showk, M.F. Paulos, D. Poland, S. Rychkov, D. Simmons-Duffin and A. Vichi, Solving the 3D Ising Model with the Conformal Bootstrap, Phys. Rev. D 86 (2012) 025022 [arXiv:1203.6064] [INSPIRE].

[13] P. Liendo, L. Rastelli and B.C. van Rees, The Bootstrap Program for Boundary $C F T_{d}$, JHEP 07 (2013) 113 [arXiv:1210.4258] [INSPIRE].

[14] S. El-Showk and M.F. Paulos, Bootstrapping Conformal Field Theories with the Extremal Functional Method, Phys. Rev. Lett. 111 (2013) 241601 [arXiv:1211.2810] [InSPIRE].

[15] F. Gliozzi, More constraining conformal bootstrap, Phys. Rev. Lett. 111 (2013) 161602 [arXiv: 1307.3111] [INSPIRE].

[16] L.F. Alday and A. Bissi, The superconformal bootstrap for structure constants, JHEP 09 (2014) 144 [arXiv: 1310.3757] [INSPIRE].

[17] D. Gaiotto, D. Mazac and M.F. Paulos, Bootstrapping the 3d Ising twist defect, JHEP 03 (2014) 100 [arXiv:1310.5078] [INSPIRE].

[18] S. El-Showk, M.F. Paulos, D. Poland, S. Rychkov, D. Simmons-Duffin and A. Vichi, Solving the $3 d$ Ising Model with the Conformal Bootstrap II. c-Minimization and Precise Critical Exponents, J. Stat. Phys. 157 (2014) 869 [arXiv:1403.4545] [INSPIRE]. 
[19] S.M. Chester, J. Lee, S.S. Pufu and R. Yacoby, The $\mathcal{N}=8$ superconformal bootstrap in three dimensions, JHEP 09 (2014) 143 [arXiv: 1406.4814] [INSPIRE].

[20] F. Kos, D. Poland and D. Simmons-Duffin, Bootstrapping Mixed Correlators in the 3D Ising Model, JHEP 11 (2014) 109 [arXiv:1406.4858] [INSPIRE].

[21] F. Caracciolo, A. Castedo Echeverri, B. von Harling and M. Serone, Bounds on OPE Coefficients in 4D Conformal Field Theories, JHEP 10 (2014) 020 [arXiv:1406.7845] [INSPIRE].

[22] M.F. Paulos, JuliBootS: a hands-on guide to the conformal bootstrap, arXiv:1412.4127 [INSPIRE].

[23] C. Beem, M. Lemos, P. Liendo, L. Rastelli and B.C. van Rees, The $\mathcal{N}=2$ superconformal bootstrap, JHEP 03 (2016) 183 [arXiv:1412.7541] [INSPIRE].

[24] D. Simmons-Duffin, A Semidefinite Program Solver for the Conformal Bootstrap, JHEP 06 (2015) 174 [arXiv: 1502.02033] [INSPIRE].

[25] N. Bobev, S. El-Showk, D. Mazac and M.F. Paulos, Bootstrapping SCFTs with Four Supercharges, JHEP 08 (2015) 142 [arXiv: 1503.02081] [INSPIRE].

[26] C. Beem, M. Lemos, L. Rastelli and B.C. van Rees, The $(2,0)$ superconformal bootstrap, Phys. Rev. D 93 (2016) 025016 [arXiv:1507.05637] [INSPIRE].

[27] L. Iliesiu, F. Kos, D. Poland, S.S. Pufu, D. Simmons-Duffin and R. Yacoby, Bootstrapping $3 D$ Fermions, JHEP 03 (2016) 120 [arXiv: 1508.00012] [INSPIRE].

[28] D. Poland and A. Stergiou, Exploring the Minimal 4D $\mathcal{N}=1$ SCFT, JHEP 12 (2015) 121 [arXiv:1509.06368] [INSPIRE].

[29] M. Lemos and P. Liendo, Bootstrapping $\mathcal{N}=2$ chiral correlators, JHEP 01 (2016) 025 [arXiv: 1510.03866] [INSPIRE].

[30] Y.-H. Lin, S.-H. Shao, D. Simmons-Duffin, Y. Wang and X. Yin, $\mathcal{N}=4$ superconformal bootstrap of the K3 CFT, JHEP 05 (2017) 126 [arXiv: 1511.04065] [INSPIRE].

[31] S.M. Chester and S.S. Pufu, Towards bootstrapping QED $D_{3}$, JHEP 08 (2016) 019 [arXiv: 1601.03476] [INSPIRE].

[32] C. Behan, PyCFTBoot: A flexible interface for the conformal bootstrap, Commun. Comput. Phys. 22 (2017) 1 [arXiv: 1602.02810] [InSPIRE].

[33] S. El-Showk and M.F. Paulos, Extremal bootstrapping: go with the flow, JHEP 03 (2018) 148 [arXiv: 1605.08087 ] [INSPIRE].

[34] Y.-H. Lin, S.-H. Shao, Y. Wang and X. Yin, $(2,2)$ superconformal bootstrap in two dimensions, JHEP 05 (2017) 112 [arXiv:1610.05371] [INSPIRE].

[35] M. Lemos, P. Liendo, C. Meneghelli and V. Mitev, Bootstrapping $\mathcal{N}=3$ superconformal theories, JHEP 04 (2017) 032 [arXiv: 1612.01536] [INSPIRE].

[36] C. Beem, L. Rastelli and B.C. van Rees, More $\mathcal{N}=4$ superconformal bootstrap, Phys. Rev. D 96 (2017) 046014 [arXiv: 1612.02363] [inSPIRE].

[37] D. Li, D. Meltzer and A. Stergiou, Bootstrapping mixed correlators in $4 D \mathcal{N}=1$ SCFTs, JHEP 07 (2017) 029 [arXiv:1702.00404] [INSPIRE]. 
[38] S. Collier, P. Kravchuk, Y.-H. Lin and X. Yin, Bootstrapping the Spectral Function: On the Uniqueness of Liouville and the Universality of BTZ, JHEP 09 (2018) 150 [arXiv: 1702.00423] [INSPIRE].

[39] M. Cornagliotto, M. Lemos and V. Schomerus, Long Multiplet Bootstrap, JHEP 10 (2017) 119 [arXiv: 1702.05101] [INSPIRE].

[40] J. Qiao and S. Rychkov, Cut-touching linear functionals in the conformal bootstrap, JHEP 06 (2017) 076 [arXiv: 1705.01357] [INSPIRE].

[41] Y. Nakayama, Bootstrap experiments on higher dimensional CFTs, Int. J. Mod. Phys. A 33 (2018) 1850036 [arXiv:1705.02744] [INSPIRE].

[42] C.-M. Chang and Y.-H. Lin, Carving Out the End of the World or (Superconformal Bootstrap in Six Dimensions), JHEP 08 (2017) 128 [arXiv: 1705.05392] [INSPIRE].

[43] A. Dymarsky, F. Kos, P. Kravchuk, D. Poland and D. Simmons-Duffin, The 3d Stress-Tensor Bootstrap, JHEP 02 (2018) 164 [arXiv:1708.05718] [INSPIRE].

[44] D. Karateev, P. Kravchuk, M. Serone and A. Vichi, Fermion Conformal Bootstrap in $4 d$, JHEP 06 (2019) 088 [arXiv: 1902.05969] [INSPIRE].

[45] L. Cornalba, Eikonal methods in AdS/CFT: Regge theory and multi-reggeon exchange, arXiv:0710.5480 [INSPIRE].

[46] L. Cornalba, M.S. Costa and J. Penedones, Deep Inelastic Scattering in Conformal QCD, JHEP 03 (2010) 133 [arXiv:0911.0043] [INSPIRE].

[47] D. Pappadopulo, S. Rychkov, J. Espin and R. Rattazzi, OPE Convergence in Conformal Field Theory, Phys. Rev. D 86 (2012) 105043 [arXiv:1208.6449] [InSPIRE].

[48] M.S. Costa, V. Goncalves and J. Penedones, Conformal Regge theory, JHEP 12 (2012) 091 [arXiv: 1209.4355] [INSPIRE].

[49] Z. Komargodski and A. Zhiboedov, Convexity and Liberation at Large Spin, JHEP 11 (2013) 140 [arXiv: 1212.4103] [INSPIRE].

[50] M. Hogervorst and S. Rychkov, Radial Coordinates for Conformal Blocks, Phys. Rev. D 87 (2013) 106004 [arXiv:1303.1111] [INSPIRE].

[51] T. Hartman, S. Jain and S. Kundu, Causality Constraints in Conformal Field Theory, JHEP 05 (2016) 099 [arXiv: 1509.00014] [INSPIRE].

[52] H. Kim, P. Kravchuk and H. Ooguri, Reflections on Conformal Spectra, JHEP 04 (2016) 184 [arXiv:1510.08772] [INSPIRE].

[53] D. Li, D. Meltzer and D. Poland, Conformal Collider Physics from the Lightcone Bootstrap, JHEP 02 (2016) 143 [arXiv: 1511.08025] [INSPIRE].

[54] Z. Komargodski, M. Kulaxizi, A. Parnachev and A. Zhiboedov, Conformal Field Theories and Deep Inelastic Scattering, Phys. Rev. D 95 (2017) 065011 [arXiv:1601.05453] [INSPIRE].

[55] T. Hartman, S. Jain and S. Kundu, A New Spin on Causality Constraints, JHEP 10 (2016) 141 [arXiv: 1601.07904 ] [INSPIRE].

[56] D.M. Hofman, D. Li, D. Meltzer, D. Poland and F. Rejon-Barrera, A Proof of the Conformal Collider Bounds, JHEP 06 (2016) 111 [arXiv:1603.03771] [INSPIRE]. 
[57] T. Hartman, S. Kundu and A. Tajdini, Averaged Null Energy Condition from Causality, JHEP 07 (2017) 066 [arXiv:1610.05308] [InSPIRE].

[58] N. Afkhami-Jeddi, T. Hartman, S. Kundu and A. Tajdini, Einstein gravity 3-point functions from conformal field theory, JHEP 12 (2017) 049 [arXiv: 1610.09378] [INSPIRE].

[59] A. Gadde, In search of conformal theories, arXiv:1702.07362 [INSPIRE].

[60] M. Hogervorst and B.C. van Rees, Crossing symmetry in alpha space, JHEP 11 (2017) 193 [arXiv: 1702.08471] [INSPIRE].

[61] S. Caron-Huot, Analyticity in Spin in Conformal Theories, JHEP 09 (2017) 078 [arXiv: 1703.00278] [INSPIRE].

[62] M. Hogervorst, Crossing Kernels for Boundary and Crosscap CFTs, arXiv:1703.08159 [INSPIRE].

[63] M. Kulaxizi, A. Parnachev and A. Zhiboedov, Bulk Phase Shift, CFT Regge Limit and Einstein Gravity, JHEP 06 (2018) 121 [arXiv:1705.02934] [INSPIRE].

[64] D. Li, D. Meltzer and D. Poland, Conformal Bootstrap in the Regge Limit, JHEP 12 (2017) 013 [arXiv: 1705. 03453] [INSPIRE].

[65] G.F. Cuomo, D. Karateev and P. Kravchuk, General Bootstrap Equations in 4D CFTs, JHEP 01 (2018) 130 [arXiv: 1705.05401] [INSPIRE].

[66] P. Dey and A. Kaviraj, Towards a Bootstrap approach to higher orders of $\epsilon$-expansion, JHEP 02 (2018) 153 [arXiv:1711.01173] [INSPIRE].

[67] D. Simmons-Duffin, D. Stanford and E. Witten, A spacetime derivation of the Lorentzian OPE inversion formula, JHEP 07 (2018) 085 [arXiv: 1711. 03816] [INSPIRE].

[68] E. Elkhidir and D. Karateev, Scalar-Fermion Analytic Bootstrap in 4D, JHEP 06 (2019) 026 [arXiv: 1712.01554] [INSPIRE].

[69] P. Kravchuk and D. Simmons-Duffin, Light-ray operators in conformal field theory, JHEP 11 (2018) 102 [arXiv: 1805.00098] [INSPIRE].

[70] D. Karateev, P. Kravchuk and D. Simmons-Duffin, Harmonic Analysis and Mean Field Theory, JHEP 10 (2019) 217 [arXiv:1809.05111] [INSPIRE].

[71] P. Liendo, Y. Linke and V. Schomerus, A Lorentzian inversion formula for defect CFT, arXiv: 1903.05222 [INSPIRE].

[72] S. Albayrak, D. Meltzer and D. Poland, More Analytic Bootstrap: Nonperturbative Effects and Fermions, JHEP 08 (2019) 040 [arXiv: 1904.00032] [INSPIRE].

[73] Z. Li, Bootstrapping Veneziano Amplitude of Vasiliev Theory and 3D Bosonization, arXiv: 1906.05834 [INSPIRE].

[74] R. Rattazzi, S. Rychkov and A. Vichi, Bounds in 4D Conformal Field Theories with Global Symmetry, J. Phys. A 44 (2011) 035402 [arXiv: 1009.5985] [INSPIRE].

[75] A. Vichi, Improved bounds for CFT's with global symmetries, JHEP 01 (2012) 162 [arXiv:1106.4037] [INSPIRE].

[76] F. Kos, D. Poland and D. Simmons-Duffin, Bootstrapping the $O(N)$ vector models, JHEP 06 (2014) 091 [arXiv: 1307.6856] [INSPIRE]. 
[77] M. Berkooz, R. Yacoby and A. Zait, Bounds on $\mathcal{N}=1$ superconformal theories with global symmetries, JHEP 08 (2014) 008 [Erratum ibid. 01 (2015) 132] [arXiv:1402.6068] [INSPIRE].

[78] Y. Nakayama and T. Ohtsuki, Approaching the conformal window of $O(n) \times O(m)$ symmetric Landau-Ginzburg models using the conformal bootstrap, Phys. Rev. D 89 (2014) 126009 [arXiv: 1404.0489] [INSPIRE].

[79] Y. Nakayama and T. Ohtsuki, Five dimensional $O(N)$-symmetric CFTs from conformal bootstrap, Phys. Lett. B 734 (2014) 193 [arXiv:1404.5201] [INSPIRE].

[80] Y. Nakayama and T. Ohtsuki, Bootstrapping phase transitions in QCD and frustrated spin systems, Phys. Rev. D 91 (2015) 021901 [arXiv: 1407.6195] [InSPIRE].

[81] J.-B. Bae and S.-J. Rey, Conformal Bootstrap Approach to O(N) Fixed Points in Five Dimensions, arXiv:1412.6549 [INSPIRE].

[82] S.M. Chester, S.S. Pufu and R. Yacoby, Bootstrapping $O(N)$ vector models in $4<d<6$, Phys. Rev. D 91 (2015) 086014 [arXiv:1412.7746] [InSPIRE].

[83] F. Kos, D. Poland, D. Simmons-Duffin and A. Vichi, Bootstrapping the $O(N)$ Archipelago, JHEP 11 (2015) 106 [arXiv:1504.07997] [INSPIRE].

[84] S.M. Chester, S. Giombi, L.V. Iliesiu, I.R. Klebanov, S.S. Pufu and R. Yacoby, Accidental Symmetries and the Conformal Bootstrap, JHEP 01 (2016) 110 [arXiv:1507.04424] [INSPIRE].

[85] S.M. Chester, L.V. Iliesiu, S.S. Pufu and R. Yacoby, Bootstrapping $O(N)$ Vector Models with Four Supercharges in $3 \leq d \leq 4$, JHEP 05 (2016) 103 [arXiv: 1511.07552] [InSPIRE].

[86] P. Dey, A. Kaviraj and K. Sen, More on analytic bootstrap for $O(N)$ models, JHEP 06 (2016) 136 [arXiv:1602.04928] [INSPIRE].

[87] Y. Nakayama, Bootstrap bound for conformal multi-flavor QCD on lattice, JHEP 07 (2016) 038 [arXiv: 1605.04052] [INSPIRE].

[88] Z. Li and N. Su, Bootstrapping Mixed Correlators in the Five Dimensional Critical $O(N)$ Models, JHEP 04 (2017) 098 [arXiv: 1607.07077] [INSPIRE].

[89] Y. Pang, J. Rong and N. Su, $\phi^{3}$ theory with $F_{4}$ flavor symmetry in $6-2 \epsilon$ dimensions: 3-loop renormalization and conformal bootstrap, JHEP 12 (2016) 057 [arXiv:1609.03007] [INSPIRE].

[90] A. Dymarsky, J. Penedones, E. Trevisani and A. Vichi, Charting the space of 3D CFTs with a continuous global symmetry, JHEP 05 (2019) 098 [arXiv: 1705.04278] [INSPIRE].

[91] A. Stergiou, Bootstrapping hypercubic and hypertetrahedral theories in three dimensions, JHEP 05 (2018) 035 [arXiv:1801.07127] [INSPIRE].

[92] S.R. Kousvos and A. Stergiou, Bootstrapping Mixed Correlators in Three-Dimensional Cubic Theories, SciPost Phys. 6 (2019) 035 [arXiv:1810.10015] [InSPIRE].

[93] A. Stergiou, Bootstrapping MN and Tetragonal CFTs in Three Dimensions, SciPost Phys. 7 (2019) 010 [arXiv: 1904.00017] [INSPIRE].

[94] L.F. Alday, A. Bissi and T. Lukowski, Large spin systematics in CFT, JHEP 11 (2015) 101 [arXiv: 1502 .07707] [INSPIRE].

[95] L.F. Alday and A. Zhiboedov, Conformal Bootstrap With Slightly Broken Higher Spin Symmetry, JHEP 06 (2016) 091 [arXiv: 1506.04659] [INSPIRE]. 
[96] L.F. Alday and A. Zhiboedov, An Algebraic Approach to the Analytic Bootstrap, JHEP 04 (2017) 157 [arXiv: 1510.08091] [inSPIRE].

[97] L.F. Alday and A. Bissi, Crossing symmetry and Higher spin towers, JHEP 12 (2017) 118 [arXiv: 1603.05150] [INSPIRE].

[98] L.F. Alday, Large Spin Perturbation Theory for Conformal Field Theories, Phys. Rev. Lett. 119 (2017) 111601 [arXiv:1611.01500] [INSPIRE].

[99] L.F. Alday, Solving CFTs with Weakly Broken Higher Spin Symmetry, JHEP 10 (2017) 161 [arXiv: 1612.00696] [INSPIRE].

[100] S. Rychkov, EPFL Lectures on Conformal Field Theory in D $\geq 3$ Dimensions, SpringerBriefs in Physics (1, 2016), 10.1007/978-3-319-43626-5 [arXiv: 1601.05000] [INSPIRE].

[101] D. Simmons-Duffin, The Conformal Bootstrap, in Theoretical Advanced Study Institute in Elementary Particle Physics: New Frontiers in Fields and Strings (TASI 2015), Boulder U.S.A. (2015), pg. 1 [arXiv: 1602.07982] [INSPIRE].

[102] D. Poland, S. Rychkov and A. Vichi, The Conformal Bootstrap: Theory, Numerical Techniques, and Applications, Rev. Mod. Phys. 91 (2019) 015002 [arXiv:1805.04405] [INSPIRE].

[103] S.M. Chester, Weizmann Lectures on the Numerical Conformal Bootstrap, arXiv: 1907.05147 [INSPIRE].

[104] F.A. Dolan and H. Osborn, Conformal four point functions and the operator product expansion, Nucl. Phys. B 599 (2001) 459 [hep-th/0011040] [INSPIRE].

[105] F.A. Dolan and H. Osborn, Conformal partial waves and the operator product expansion, Nucl. Phys. B 678 (2004) 491 [hep-th/0309180] [INSPIRE].

[106] S. Ferrara, A.F. Grillo, G. Parisi and R. Gatto, Covariant expansion of the conformal four-point function, Nucl. Phys. B 49 (1972) 77 [Erratum ibid. 53 (1973) 643] [INSPIRE].

[107] S. Ferrara, A.F. Grillo, R. Gatto and G. Parisi, Analyticity properties and asymptotic expansions of conformal covariant green's functions, Nuovo Cim. A 19 (1974) 667 [INSPIRE].

[108] V.K. Dobrev, G. Mack, V.B. Petkova, S.G. Petrova and I.T. Todorov, Harmonic Analysis on the n-Dimensional Lorentz Group and Its Application to Conformal Quantum Field Theory, Lect. Notes Phys. 63 (1977) 1.

[109] H. Exton, On the system of partial differential equations associated with Appell's function F4, J. Phys. A 28 (1995) 631.

[110] S. Giombi, S. Prakash and X. Yin, A Note on CFT Correlators in Three Dimensions, JHEP 07 (2013) 105 [arXiv:1104.4317] [INSPIRE].

[111] M.S. Costa, J. Penedones, D. Poland and S. Rychkov, Spinning Conformal Correlators, JHEP 11 (2011) 071 [arXiv:1107.3554] [INSPIRE].

[112] F.A. Dolan and H. Osborn, Conformal Partial Waves: Further Mathematical Results, arXiv:1108.6194 [INSPIRE].

[113] M.S. Costa, J. Penedones, D. Poland and S. Rychkov, Spinning Conformal Blocks, JHEP 11 (2011) 154 [arXiv:1109.6321] [InSPIRE].

[114] D. Simmons-Duffin, Projectors, Shadows, and Conformal Blocks, JHEP 04 (2014) 146 [arXiv: 1204.3894] [INSPIRE]. 
[115] M.S. Costa and T. Hansen, Conformal correlators of mixed-symmetry tensors, JHEP 02 (2015) 151 [arXiv: 1411.7351] [inSPIRE].

[116] E. Elkhidir, D. Karateev and M. Serone, General Three-Point Functions in $4 D$ CFT, JHEP 01 (2015) 133 [arXiv: 1412.1796] [INSPIRE].

[117] A. Castedo Echeverri, E. Elkhidir, D. Karateev and M. Serone, Deconstructing Conformal Blocks in 4D CFT, JHEP 08 (2015) 101 [arXiv: 1505.03750] [INSPIRE].

[118] E. Hijano, P. Kraus, E. Perlmutter and R. Snively, Witten Diagrams Revisited: The AdS Geometry of Conformal Blocks, JHEP 01 (2016) 146 [arXiv: 1508.00501] [INSPIRE].

[119] F. Rejon-Barrera and D. Robbins, Scalar-Vector Bootstrap, JHEP 01 (2016) 139 [arXiv: 1508.02676] [INSPIRE].

[120] J. Penedones, E. Trevisani and M. Yamazaki, Recursion Relations for Conformal Blocks, JHEP 09 (2016) 070 [arXiv: 1509.00428] [INSPIRE].

[121] L. Iliesiu, F. Kos, D. Poland, S.S. Pufu, D. Simmons-Duffin and R. Yacoby, Fermion-Scalar Conformal Blocks, JHEP 04 (2016) 074 [arXiv: 1511.01497] [INSPIRE].

[122] A. Castedo Echeverri, E. Elkhidir, D. Karateev and M. Serone, Seed Conformal Blocks in 4D CFT, JHEP 02 (2016) 183 [arXiv:1601.05325] [INSPIRE].

[123] M. Isachenkov and V. Schomerus, Superintegrability of d-dimensional Conformal Blocks, Phys. Rev. Lett. 117 (2016) 071602 [arXiv: 1602.01858] [INSPIRE].

[124] M.S. Costa, T. Hansen, J. Penedones and E. Trevisani, Projectors and seed conformal blocks for traceless mixed-symmetry tensors, JHEP 07 (2016) 018 [arXiv: 1603.05551] [INSPIRE].

[125] M.S. Costa, T. Hansen, J. Penedones and E. Trevisani, Radial expansion for spinning conformal blocks, JHEP 07 (2016) 057 [arXiv: 1603.05552] [INSPIRE].

[126] H.-Y. Chen and J.D. Qualls, Quantum Integrable Systems from Conformal Blocks, Phys. Rev. D 95 (2017) 106011 [arXiv: 1605.05105] [INSPIRE].

[127] M. Nishida and K. Tamaoka, Geodesic Witten diagrams with an external spinning field, PTEP 2017 (2017) 053B06 [arXiv: 1609.04563] [INSPIRE].

[128] C. Cordova, T.T. Dumitrescu and K. Intriligator, Multiplets of Superconformal Symmetry in Diverse Dimensions, JHEP 03 (2019) 163 [arXiv: 1612.00809] [INSPIRE].

[129] V. Schomerus, E. Sobko and M. Isachenkov, Harmony of Spinning Conformal Blocks, JHEP 03 (2017) 085 [arXiv: 1612.02479] [INSPIRE].

[130] P. Kravchuk and D. Simmons-Duffin, Counting Conformal Correlators, JHEP 02 (2018) 096 [arXiv: 1612.08987] [INSPIRE].

[131] F. Gliozzi, A.L. Guerrieri, A.C. Petkou and C. Wen, The analytic structure of conformal blocks and the generalized Wilson-Fisher fixed points, JHEP 04 (2017) 056 [arXiv: 1702.03938] [INSPIRE].

[132] A. Castro, E. Llabrés and F. Rejon-Barrera, Geodesic Diagrams, Gravitational Interactions \& OPE Structures, JHEP 06 (2017) 099 [arXiv: 1702.06128] [INSPIRE].

[133] E. Dyer, D.Z. Freedman and J. Sully, Spinning Geodesic Witten Diagrams, JHEP 11 (2017) 060 [arXiv: 1702.06139] [INSPIRE].

[134] C. Sleight and M. Taronna, Spinning Witten Diagrams, JHEP 06 (2017) 100 [arXiv: 1702.08619] [INSPIRE]. 
[135] H.-Y. Chen, E.-J. Kuo and H. Kyono, Anatomy of Geodesic Witten Diagrams, JHEP 05 (2017) 070 [arXiv: 1702.08818] [INSPIRE].

[136] S. Pasterski and S.-H. Shao, Conformal basis for flat space amplitudes, Phys. Rev. D 96 (2017) 065022 [arXiv: 1705.01027] [inSPIRE].

[137] V. Cardoso, T. Houri and M. Kimura, Mass Ladder Operators from Spacetime Conformal Symmetry, Phys. Rev. D 96 (2017) 024044 [arXiv:1706.07339] [INSPIRE].

[138] D. Karateev, P. Kravchuk and D. Simmons-Duffin, Weight Shifting Operators and Conformal Blocks, JHEP 02 (2018) 081 [arXiv:1706.07813] [INSPIRE].

[139] P. Kravchuk, Casimir recursion relations for general conformal blocks, JHEP 02 (2018) 011 [arXiv: 1709.05347] [INSPIRE].

[140] P. Dey, K. Ghosh and A. Sinha, Simplifying large spin bootstrap in Mellin space, JHEP 01 (2018) 152 [arXiv: 1709.06110] [INSPIRE].

[141] S. Hollands, Action principle for OPE, Nucl. Phys. B 926 (2018) 614 [arXiv:1710.05601] [INSPIRE].

[142] V. Schomerus and E. Sobko, From Spinning Conformal Blocks to Matrix Calogero-Sutherland Models, JHEP 04 (2018) 052 [arXiv: 1711.02022] [INSPIRE].

[143] M. Isachenkov and V. Schomerus, Integrability of conformal blocks. Part I. Calogero-Sutherland scattering theory, JHEP 07 (2018) 180 [arXiv:1711.06609] [INSPIRE].

[144] J. Faller, S. Sarkar and M. Verma, Mellin Amplitudes for Fermionic Conformal Correlators, JHEP 03 (2018) 106 [arXiv:1711.07929] [INSPIRE].

[145] J. Rong and N. Su, Scalar CFTs and Their Large N Limits, JHEP 09 (2018) 103 [arXiv: 1712.00985] [INSPIRE].

[146] H.-Y. Chen, E.-J. Kuo and H. Kyono, Towards Spinning Mellin Amplitudes, Nucl. Phys. B 931 (2018) 291 [arXiv:1712.07991] [InSPIRE].

[147] C. Sleight and M. Taronna, Spinning Mellin Bootstrap: Conformal Partial Waves, Crossing Kernels and Applications, Fortsch. Phys. 66 (2018) 1800038 [arXiv:1804.09334] [InSPIRE].

[148] M.S. Costa and T. Hansen, AdS Weight Shifting Operators, JHEP 09 (2018) 040 [arXiv: 1805.01492] [INSPIRE].

[149] N. Kobayashi and T. Nishioka, Spinning conformal defects, JHEP 09 (2018) 134 [arXiv: 1805.05967] [INSPIRE].

[150] A. Bhatta, P. Raman and N.V. Suryanarayana, Scalar Blocks as Gravitational Wilson Networks, JHEP 12 (2018) 125 [arXiv: 1806.05475] [INSPIRE].

[151] E. Lauria, M. Meineri and E. Trevisani, Spinning operators and defects in conformal field theory, JHEP 08 (2019) 066 [arXiv: 1807.02522] [INSPIRE].

[152] J. Liu, E. Perlmutter, V. Rosenhaus and D. Simmons-Duffin, d-dimensional SYK, AdS Loops, and 6j Symbols, JHEP 03 (2019) 052 [arXiv:1808.00612] [INSPIRE].

[153] N. Gromov, V. Kazakov and G. Korchemsky, Exact Correlation Functions in Conformal Fishnet Theory, JHEP 08 (2019) 123 [arXiv:1808.02688] [INSPIRE].

[154] V. Rosenhaus, Multipoint Conformal Blocks in the Comb Channel, JHEP 02 (2019) 142 [arXiv: 1810.03244] [INSPIRE]. 
[155] X. Zhou, Recursion Relations in Witten Diagrams and Conformal Partial Waves, JHEP 05 (2019) 006 [arXiv: 1812.01006] [INSPIRE].

[156] V. Kazakov, E. Olivucci and M. Preti, Generalized fishnets and exact four-point correlators in chiral $\mathrm{CFT}_{4}$, JHEP 06 (2019) 078 [arXiv:1901.00011] [INSPIRE].

[157] W. Li, Closed-form expression for cross-channel conformal blocks near the lightcone, JHEP 01 (2020) 055 [arXiv: 1906.00707] [INSPIRE].

[158] V. Gonçalves, R. Pereira and X. Zhou, $20^{\prime}$ Five-Point Function from $A d S_{5} \times S^{5}$ Supergravity, JHEP 10 (2019) 247 [arXiv:1906.05305] [INSPIRE].

[159] C.B. Jepsen and S. Parikh, Propagator identities, holographic conformal blocks, and higher-point AdS diagrams, JHEP 10 (2019) 268 [arXiv:1906.08405] [INSPIRE].

[160] P.A.M. Dirac, Wave equations in conformal space, Annals Math. 37 (1936) 429 [INSPIRE].

[161] G. Mack and A. Salam, Finite component field representations of the conformal group, Annals Phys. 53 (1969) 174 [INSPIRE].

[162] S. Weinberg, Six-dimensional Methods for Four-dimensional Conformal Field Theories, Phys. Rev. D 82 (2010) 045031 [arXiv: 1006.3480] [INSPIRE].

[163] S. Weinberg, Six-dimensional Methods for Four-dimensional Conformal Field Theories II: Irreducible Fields, Phys. Rev. D 86 (2012) 085013 [arXiv:1209.4659] [INSPIRE].

[164] S. Ferrara, A.F. Grillo and R. Gatto, Manifestly conformal covariant operator-product expansion, Lett. Nuovo Cim. 2S2 (1971) 1363 [INSPIRE].

[165] S. Ferrara, R. Gatto and A.F. Grillo, Conformal invariance on the light cone and canonical dimensions, Nucl. Phys. B 34 (1971) 349 [INSPIRE].

[166] S. Ferrara, A.F. Grillo and R. Gatto, Manifestly conformal-covariant expansion on the light cone, Phys. Rev. D 5 (1972) 3102 [InSPIRE].

[167] S. Ferrara, P. Gatto and A.F. Grilla, Conformal algebra in space-time and operator product expansion, Springer Tracts Mod. Phys. 67 (1973) 1 [INSPIRE].

[168] V.K. Dobrev, V.B. Petkova, S.G. Petrova and I.T. Todorov, Dynamical Derivation of Vacuum Operator Product Expansion in Euclidean Conformal Quantum Field Theory, Phys. Rev. D 13 (1976) 887 [INSPIRE].

[169] G. Mack, Convergence of Operator Product Expansions on the Vacuum in Conformal Invariant Quantum Field Theory, Commun. Math. Phys. 53 (1977) 155 [inSPIRE].

[170] J.-F. Fortin and W. Skiba, Conformal Bootstrap in Embedding Space, Phys. Rev. D 93 (2016) 105047 [arXiv : 1602 .05794] [inSPIRE].

[171] J.-F. Fortin and W. Skiba, Conformal Differential Operator in Embedding Space and its Applications, JHEP 07 (2019) 093 [arXiv: 1612.08672] [INSPIRE].

[172] V. Comeau, J.-F. Fortin and W. Skiba, Further Results on a Function Relevant for Conformal Blocks, arXiv: 1902.08598 [INSPIRE].

[173] J.-F. Fortin, V. Prilepina and W. Skiba, Conformal two-point correlation functions from the operator product expansion, JHEP 04 (2020) 114 [arXiv: 1906.12349] [INSPIRE].

[174] J.-F. Fortin, V. Prilepina and W. Skiba, Conformal Three-Point Correlation Functions from the Operator Product Expansion, arXiv:1907.08599 [INSPIRE].

[175] F.A. Dolan and H. Osborn, Implications of $N=1$ superconformal symmetry for chiral fields, Nucl. Phys. B 593 (2001) 599 [hep-th/0006098] [INSPIRE]. 